University of Tennessee Health Science Center UTHSC Digital Commons

$12-2008$

\title{
Role of the LPA2 Receptor in Protecting Against Apoptosis
}

Shuyu $\mathrm{E}$

University of Tennessee Health Science Center

Follow this and additional works at: https://dc.uthsc.edu/dissertations

Part of the Medical Physiology Commons

\section{Recommended Citation}

E, Shuyu , "Role of the LPA2 Receptor in Protecting Against Apoptosis" (2008). Theses and Dissertations (ETD). Paper 336. http://dx.doi.org/10.21007/etd.cghs.2008.0079.

This Dissertation is brought to you for free and open access by the College of Graduate Health Sciences at UTHSC Digital Commons. It has been accepted for inclusion in Theses and Dissertations (ETD) by an authorized administrator of UTHSC Digital Commons. For more information, please contact jwelch30@uthsc.edu. 


\title{
Role of the LPA2 Receptor in Protecting Against Apoptosis
}

\begin{abstract}
Lysophosphatidic acid (LPA) is a naturally occurring lipid mediator. It exists abundantly in biological fluids such as serum, saliva, follicular fluid, seminal fluid and malignant effusions and induces a vast array of biological responses affecting cell growth, survival, differentiation, migration and morphology. We recently identified lysophosphatidic acid (LPA) as a potent antiapoptotic agent for the intestinal epithelium. Based on computational modeling octadecenyl thiophosphate (OTP) was synthesized: a novel rationally designed, metabolically stabilized LPA mimic. OTP was more efficacious than LPA in reducing girradiation-, camptothecin-, or TNF-a/何cycloheximide-induced apoptosis and caspase 3, 8 and 9 activity in the IEC- 6 cell line. The OTP- and LPA-elicited antiapoptotic effects were completely blocked by the MEK inhibitor PD98059 and the PI3K inhibitor LY294002. Pertussis toxin partially abolished OTP-induced ERK1/2 and apoptotic protection. In RH7777 cells lacking LPA receptors, OTP selectively protected LPA2 but not LPA 1 and LPA 3 transfectants. In C57BL/ 6 and LPA 1 knockout mice exposed to 15 Gy g-irradiation, orally applied OTP reduced the number of apoptotic bodies and activated caspase 3 positive cells but was ineffective in LPA 2 knockouts. OTP, with higher efficacy than LPA, enhanced intestinal crypt survival in C57BL/6 mice but had no effect in LPA2 knockouts. Intraperitonealy administered OTP reduced death caused by LD $100 / 30$ radiation by $50 \%$. Our data indicate that OTP is a highly effective antiapoptotic agent that engages similar prosurvival pathways to LPA through the $\mathrm{LPA}_{2}$ receptor subtype.

Unique sequence motifs in the LPA2 carboxyl-terminal (CT) enable it to form macromolecular complexes with PDZ and LIM domain proteins, which link it to $G$ protein-independent signaling networks. Using deletion and site-specific mutagenesis, we mapped out C311xxC314 motif of LPA $2-C T$ that is required for interaction with LIM domain proteins thyroid hormone receptor interactive protein 6 (TRIP6) and the proapoptotic molecule Siva-1 in vitro and in vivo. Palmitoylation that occurs on these cysteine residues, however, did not affect the association with TRIP6 or Siva-1. The L351A mutation in the PDZ motif weakened but did not abolish interaction with LIM proteins. Alanine mutation of the LIM binding motif or PDZ domain binding motif attenuated LPA-induced activation of the prosurvival ERK $1 / 2$ and Akt pathways in mouse embryonic fibroblasts (MEF) derived from LPA 1 and LPA 2 double knockout mice and reconstituted with mutants of $\mathrm{LPA}_{2}$. Neither of these mutations alone nor in combination had a detectable effect on G-protein-linked activation of $\mathrm{Ca}^{2+}$ mobilization. Triple alanine mutations modifying residues Cys311, Cys314, and Leu351 abolished the antiapoptotic effect of LPA. Together, these findings suggest the macromolecular complex formed between LPA2, TRIP6/Siva-1, and PDZ domain proteins plays an important role in mediating the anti-apoptotic effects of LPA2.
\end{abstract}

\section{Document Type}

Dissertation

Degree Name

Doctor of Philosophy (PhD)

Program

Physiology

\section{Research Advisor}

Gabor Tigyi, M.D., Ph.D. 


\section{Keywords}

LPA, LPA2 receptor, apoptosis, irradiation, protein-protein interaction, LIM domain PDZ domain

\section{Subject Categories}

Medical Physiology | Medical Sciences | Medicine and Health Sciences 
ROLE OF THE LPA 2 RECEPTOR IN PROTECTING AGAINST APOPTOSIS

\author{
A Dissertation \\ Presented for \\ The Graduate Studies Council \\ The University of Tennessee \\ Health Science Center
}

In Partial Fulfillment

Of the Requirements for the Degree

Doctor of Philosophy

From The University of Tennessee

By

Shuyu E

December, 2008 
Chapter 2 (C) 2007 by the American Gastroenterological Association Institute. All other material (C) 2008 by Shuyu E. 


\section{DEDICATION}

This dissertation is dedicated to my husband $\mathrm{Xu}$ Liu

for his endless love and support.

and to my children

Eilene Liu

Nolan Liu 


\section{ACKNOWLEDGEMENTS}

I am greatly indebted to my supervisor, Dr. Gabor Tigyi, who accepted me into his lab while I was relocating two years ago. The training in his lab greatly benefited me in both science and my personal life. Being exposed to his multi-dimensional lab and broad research capability, I was trained to have an open mind. During this time I regained my confidence, which is critical for my success. In retrospect, I cherished the two years spent in his lab. I am also grateful to all the members in his lab: Billy Walentine, Yuko Fujiwara, Tamotsu Tsukahara, Ryoko Tsukahara, Alyssa Bolen, Daniel Osborne, Huazhang Guo and Dianna Liu. In such a united family, I received support anytime I needed it. This environment made it enjoyable to work everyday. Also, I want to thank Dr. Wenlin Deng for invaluable help in apoptosis studies and Dr. Junming Yue for lentiviral transduction and making stable MEFs.

In particular, I want to thank Dr. Fannie Lin who was the collaborator of my project. It was an exciting and inspiring experience to work with her. She is the most energetic, enthusiastic, persevering and intelligent scientist I have ever known. I would like to acknowledge that Fig.3.3, Fig.3.4, Fig.3.5, Fig.3.6, Fig3.8, Fig3.9, Fig3.12, Fig3.16, Fig.3.19, and Fig.3.21 were done in collaboration with her. Also thanks go to Yun-Ju Lai, and Chen-Shan Chen from her lab for providing us the constructs and help in some of the CoIP experiments.

I acknowledge Dr. Akio Kihara for his work on the palmitoylation study.

I would also like to sincerely thank the other committee members: Dr. Hofmann, Dr. Waters, Dr. Naren, and Dr. Parrill for always being understanding and providing valuable and critical suggestions.

I would also like to thank Dr. Schneider, Dr. Watsky, Dr. Hofmann, and Dr. Leffler, whose support encouraged me to continue my Ph.D. in spite of my setback that forced me to change mentors after four years and resulted in a lack of confidence.

Also, I could not forget the help from Dr. Johnson's lab members: Dr. Shi Jin and Dr. Ramsey Ray.

I also want to express my gratitude to my parents, mother-in-law, and sister-inlaw for their support and helping me care for my daughter, which allowed me time to concentrate on my work.

I greatly appreciate the support from Gerwin Scholarship.

I appreciate the permission of reuse of the illustrations from Radiation Research Society and Nature Publishing Group. 


\begin{abstract}
Lysophosphatidic acid (LPA) is a naturally occurring lipid mediator. It exists abundantly in biological fluids such as serum, saliva, follicular fluid, seminal fluid and malignant effusions and induces a vast array of biological responses affecting cell growth, survival, differentiation, migration and morphology. We recently identified lysophosphatidic acid (LPA) as a potent antiapoptotic agent for the intestinal epithelium. Based on computational modeling octadecenyl thiophosphate (OTP) was synthesized: a novel rationally designed, metabolically stabilized LPA mimic. OTP was more efficacious than LPA in reducing $\gamma$-irradiation-, camptothecin-, or TNF$\alpha /$ cycloheximide-induced apoptosis and caspase 3, 8 and 9 activity in the IEC-6 cell line. The OTP- and LPA-elicited antiapoptotic effects were completely blocked by the MEK inhibitor PD98059 and the PI3K inhibitor LY294002. Pertussis toxin partially abolished OTP-induced ERK1/2 and apoptotic protection. In RH7777 cells lacking LPA receptors, OTP selectively protected LPA 2 but not $\mathrm{LPA}_{1}$ and $\mathrm{LPA}_{3}$ transfectants. In C57BL/6 and $\mathrm{LPA}_{1}$ knockout mice exposed to $15 \mathrm{~Gy} \gamma$-irradiation, orally applied OTP reduced the number of apoptotic bodies and activated caspase 3 positive cells but was ineffective in LPA $_{2}$ knockouts. OTP, with higher efficacy than LPA, enhanced intestinal crypt survival in $\mathrm{C} 57 \mathrm{BL} / 6$ mice but had no effect in $\mathrm{LPA}_{2}$ knockouts. Intraperitonealy administered OTP reduced death caused by $\mathrm{LD}_{100 / 30}$ radiation by $50 \%$. Our data indicate that OTP is a highly effective antiapoptotic agent that engages similar prosurvival pathways to LPA through the $\mathrm{LPA}_{2}$ receptor subtype.

Unique sequence motifs in the $\mathrm{LPA}_{2}$ carboxyl-terminal (CT) enable it to form macromolecular complexes with PDZ and LIM domain proteins, which link it to G protein-independent signaling networks. Using deletion and site-specific mutagenesis, we mapped out $\mathrm{C} 311 \times x \mathrm{C} 314$ motif of $\mathrm{LPA}_{2}-\mathrm{CT}$ that is required for interaction with LIM domain proteins thyroid hormone receptor interactive protein 6 (TRIP6) and the proapoptotic molecule Siva-1 in vitro and in vivo. Palmitoylation that occurs on these cysteine residues, however, did not affect the association with TRIP6 or Siva-1. The L351A mutation in the PDZ motif weakened but did not abolish interaction with LIM proteins. Alanine mutation of the LIM binding motif or PDZ domain binding motif attenuated LPA-induced activation of the prosurvival ERK1/2 and Akt pathways in mouse embryonic fibroblasts (MEF) derived from $\mathrm{LPA}_{1}$ and $\mathrm{LPA}_{2}$ double knockout mice and reconstituted with mutants of $\mathrm{LPA}_{2}$. Neither of these mutations alone nor in combination had a detectable effect on G-protein-linked activation of $\mathrm{Ca}^{2+}$ mobilization. Triple alanine mutations modifying residues Cys311, Cys314, and Leu351 abolished the antiapoptotic effect of LPA. Together, these findings suggest the macromolecular complex formed between $\mathrm{LPA}_{2}$, TRIP6/Siva-1, and PDZ domain proteins plays an important role in mediating the anti-apoptotic effects of $\mathrm{LPA}_{2}$.
\end{abstract}




\section{TABLE OF CONTENTS}

CHAPTER 1. GENERAL INTRODUCTION_........................................................1

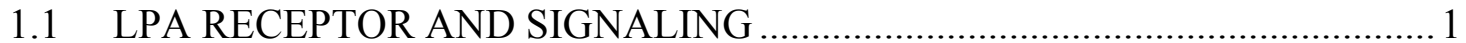

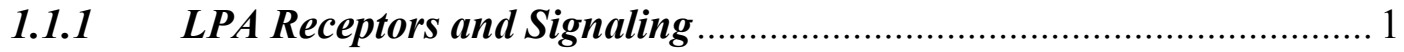

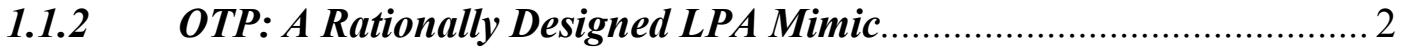

1.2 APOPTOSIS IN SMALL INTESTINE EPITHELIUM …………………......... 3

1.2.1 Intrinsic (Mitochondria-mediated) Apoptotic Pathway …...................... 3

1.2.2 Extrinsic (Death Receptor-mediated) Apoptotic Pathway .................... 6

1.2.3 Physiology of Small Intestinal Stem Cells ...................................... 6

1.2.4 Radiobiology in Mouse Small Intestinal Stem Cells .......................... 8

1.3 LIM AND PDZ DOMAIN PROTEINS INTERACTING WITH LPA $\mathrm{C}_{2}$ -

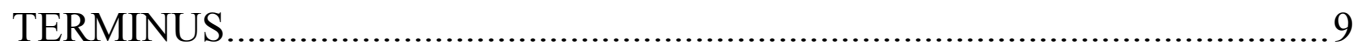

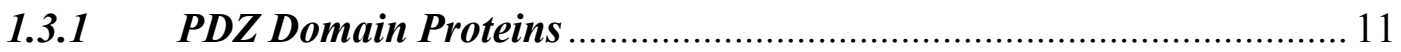

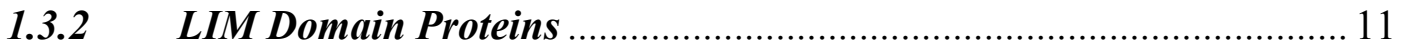

\section{CHAPTER 2. THE LYSOPHOSPHATIDIC ACID TYPE 2 RECEPTOR} IS REQUIRED FOR PROTECTING AGAINST RADIATION-INDUCED

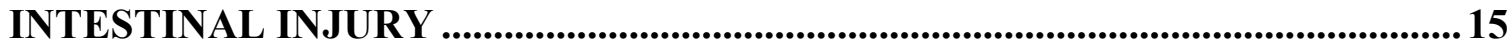

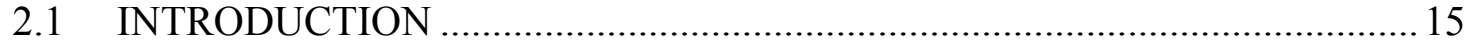

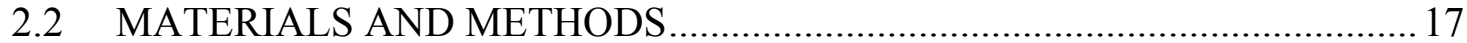

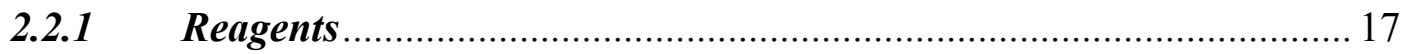

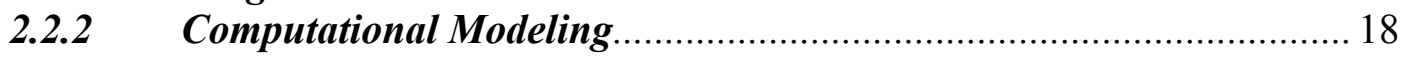

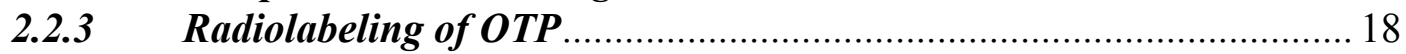

2.2.4 Determining OTP Absorption from the Gut................................... 18

2.2.5 Assaying OTP Metabolism by Pancreatic Lipase and Lipid Phosphate Phosphatase 1 (LPP1) .................................................. 20

2.2.6 Cell Culture and Induction of Apoptosis In Vitro............................. 20

2.2.7 Pharmacological Characterization of OTP ……….......................... 20

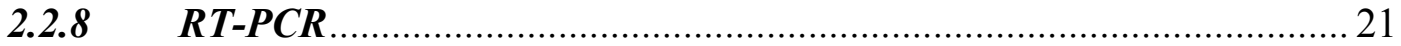

2.2.9 Evaluating Apoptosis by DNA Fragmentation and Caspase Activity . 21

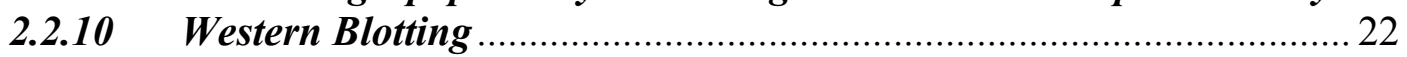

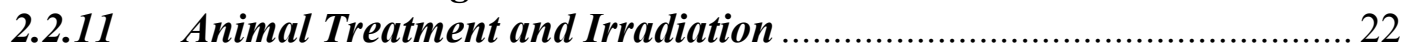

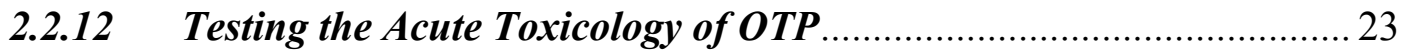

2.2.13 Assessing Apoptosis and Crypt Survival in Intestine ……..................23

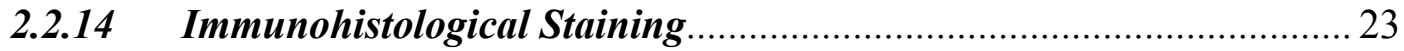

2.2.15 Monitoring the Radiation-Mitigating Effect of OTP on Survival ....... 24

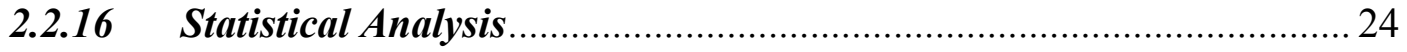

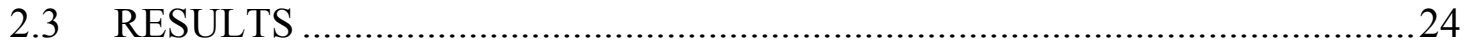

2.3.1 Molecular Modeling and Pharmacological Characterization of

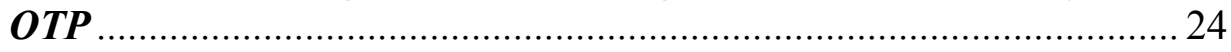

2.3.2 Metabolic Resistance of OTP to Pancreatic Lipase and Lipid Phosphate Phosphatase 1............................................................ 25

2.3.3 Absorption of Orally Applied OTP into the Blood ……….................. 25

2.3.4 Orally Applied OTP Is Non-toxic in the Effective Dose Range........... 26 
2.3.5 OTP Surpasses the Antiapoptotic Activity of LPA in IEC-6 Cells ...... 26

2.3.6 The Antiapoptotic Activity of OTP Requires PTX-sensitive G Protein, Mitogen Activated Protein Kinase and PI3-Kinase/AKT Signaling

2.3.7 OTP Selectively Protects LPA $A_{2}$ Transfectants from TNF- $\alpha$-induced

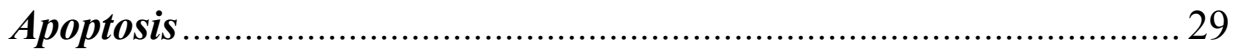

2.3.8 OTP Attenuates Radiation-induced Apoptosis in the Intestine ........... 34

2.3.9 OTP and LPA Enhance Intestinal Crypt Survival after Radiation

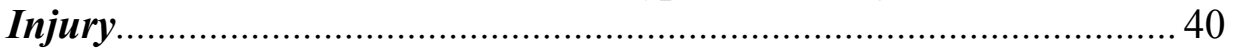

2.3.10 OTP Reduces Radiation-induced Death ........................................ 45

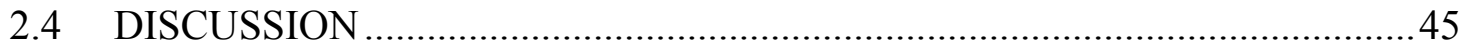

CHAPTER 3. INTERACTION OF LPA, WITH LIM AND PDZ DOMAIN
PROTEINS MEDIATES ITS PROTECTION AGAINST APOPTOSIS ................. 49

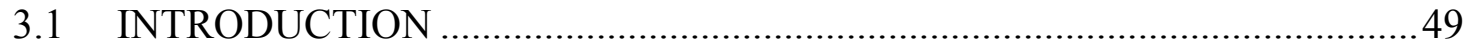

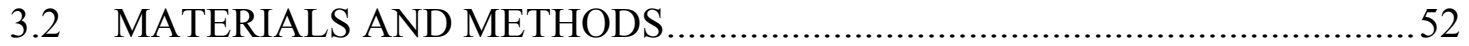

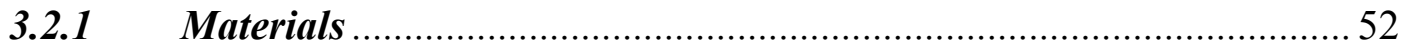

3.2.2 Plasmid Construction and Site-directed Mutagenesis ........................ 52

3.2.3 Cell Culture and Transfection ............................................................. 56

3.2.4 Construction of LPA $A_{2}$-Lentiviral Vectors and Production of

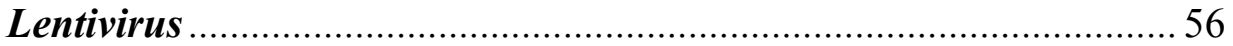

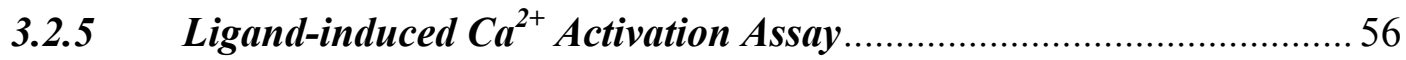

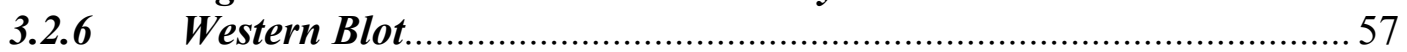

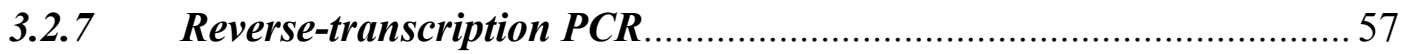

3.2.8 Quantitative Real Time-PCR …..................................................... 58

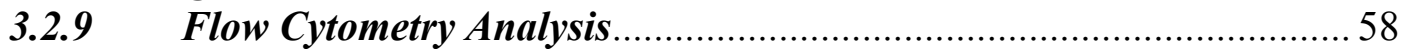

3.2.10 Cellular Co-immunoprecipitation and GST Pull-down .....................59

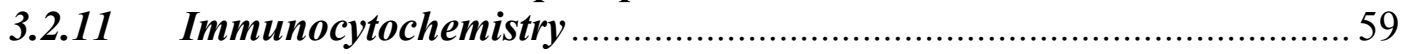

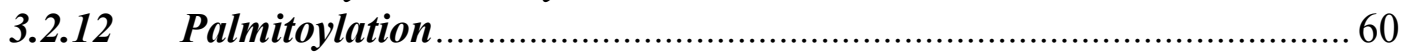

3.2.13 Acyl-Biotinyl Exchange Method ........................................................ 60

3.2.14 Adriamycin-induced Siva-1 Expression, Procaspase-3 Cleavage

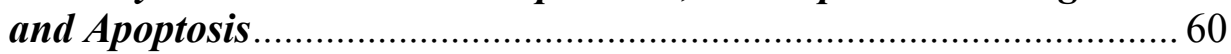

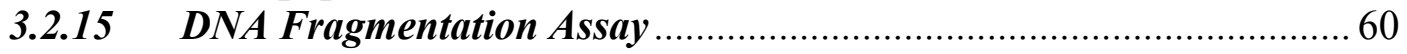

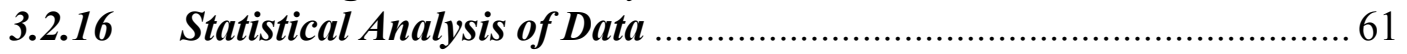

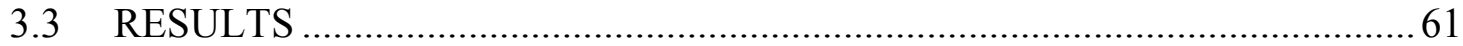

3.3.1 Spaced Cysteine Residues in LPA $A_{2}-C T$ May Constitute Potential Zinc-finger Binding Structure to TRIP6/Siva-1 ................................. 61

3.3.2 TRIP6 and Siva-1 Binding Motif Is Mapped to aa309-316 of LPA2-

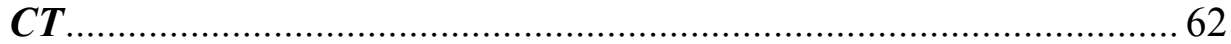

3.3.3 The C311xxC314 Motif of the LPA $\mathrm{A}_{2}$ Receptor Is Required for Interaction with TRIPG and Siva-1.

3.3.4 LPA Receptor Directly Interacts with TRIP6 and Siva-1 via the C311xxC314 Motif.

3.3.5 Palmitoylation Does Not Affect the Interaction of $\mathrm{LPA}_{2}$ with TRIP6/Siva-1 
3.3.6 Characterization of Lentivirus-transduced Stable MEFs Expressing $h L P A_{2} W T$ and Mutants Defective in Interaction with PDZ or LIM Domain Proteins.

3.3.7 LPA $\quad$-mediated Protection from DNA Damage-induced Apoptosis

Is Regulated by the CxxC and PDZ-binding Motifs .......................... 81

3.4 DISCUSSION

LIST OF REFERENCES .........................................................................................90

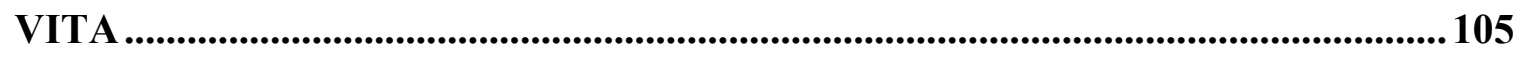




\section{LIST OF FIGURES}

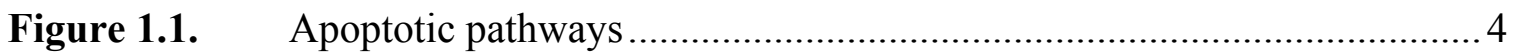

Figure 1.2. Current model for the cell lineage in small intestinal crypts....................

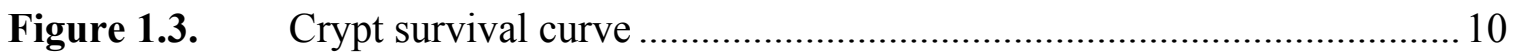

Figure 1.4. Conserved sequence and topology of the LIM domain...........................12

Figure 2.1. Structure, computational modeling and synthesis of OTP ......................19

Figure 2.2. OTP is more effective in reducing apoptosis than LPA in IEC-6 cells.. 27

Figure 2.3. Activation of prosurvival signaling pathways by LPA and OTP in IEC-6 cells

Figure 2.4. OTP selectively protects $\mathrm{LPA}_{2}$ transfectants against TNF- $\alpha / \mathrm{CHX}$ induced apoptosis

Figure 2.5. OTP inhibits apoptosis in small intestinal epithelium of mice following $\gamma$-irradiation

Figure 2.6. OTP and LPA reduces caspase 3 activation and activates prosurvival pathways in vivo

Figure 2.7. Immunohistochemical localization of antiapoptotic Bcl- $\mathrm{X}_{\mathrm{L}}$ protein in vehicle (200 $\mu \mathrm{M}$ BSA), LPA and OTP-pretreated, irradiated small intestinal sections of $\mathrm{C} 57 \mathrm{BL} / 6$ mice

Figure 2.8. Clonogenic assays reveals increased intestinal crypt survival in OTPand LPA-treated mice following irradiation

Figure 2.9. Representive $H \& E$ stained gut cross-sections from treated $L P A_{1}$ and $\mathrm{LPA}_{2} \mathrm{KO}$ mice sacrified 4 days after irradiation

Figure 2.10. OTP administered intraperitoneally 30 minutes prior to irradiation with a 9 Gy dose $\left(\mathrm{LD}_{100 / 30}\right)$ increased 30-day survival.

Figure 3.1. Amino acid sequence alignment of human Siva-1, TRIP6 and LPA receptor-CTs

Figure 3.2. Schematic illustration of the $\mathrm{LPA}_{2}-\mathrm{CT}$ deletion constructs 62

Figure 3.3. TRIP6 binding domain is localized between aa309-316 of $\mathrm{LPA}_{2}-\mathrm{CT} \ldots 63$ 
Figure 3.4. Siva-1 binding domain is located between aa309-316 of $\mathrm{LPA}_{2}-\mathrm{CT}$...... 64

Figure 3.5. The $\mathrm{C} 311-\mathrm{x}-\mathrm{x}-\mathrm{C} 314$ motif located at the proximal region of the carboxyl terminal tail of the $\mathrm{LPA}_{2}$ receptor regulates the cellular interaction with TRIP6 and Siva-1

Figure 3.6. Mutation of both Cys 311 and Cys 314 to Alanine abolishes the colocaliztion of $\mathrm{LPA}_{2}$ with Siva-1

Figure 3.7. Cellular expression of $\mathrm{LPA}_{2}-\mathrm{CT}$ point mutants in $\mathrm{RH}$ cells. .68

Figure 3.8. Alanine scanning of aa296-316 by coimmunoprecipitation with TRIP6 .70

Figure 3.9. Mutation of $\mathrm{LPA}_{2}-\mathrm{CT}$ Cys 311 or Cys 314 to Alanine 7 abolishes its direct binding to TRIP6; however, only when both are mutated binding to Siva-1 is completely eliminated

Figure 3.10. Mutation of $\mathrm{LPA}_{2}-\mathrm{CT}$ cysteine residues inhibits receptor palmitoylation

Figure 3.11. 2-bromopalmitate inhibits $\mathrm{LPA}_{2}$ palmitoylation

Figure 3.12. Palmitoylation is not required for interaction of $\mathrm{LPA}_{2}-\mathrm{CT}$ with TRIP6 or Siva-1

Figure 3.13. Schematic illustration of lentiviral $\mathrm{LPA}_{2}$ constructs .77

Figure 3.14. mRNA expression profile of LPA receptors in spontaneously immortalized $\mathrm{LPA}_{1 / 2}$ DKO MEFs

Figure 3.15. mRNA expression of WT $\mathrm{LPA}_{2}$ and its mutants in lentivirustransduced DKO MEFs .78

Figure 3.16. Cellular protein and cell surface expression of WT $\mathrm{LPA}_{2}$ and its mutants in lentivirus-transduced DKO MEFs

Figure 3.17. Mutation of $\mathrm{CxxC}$ and/or PDZ motif(s) of $\mathrm{LPA}_{2}-\mathrm{CT}$ does not affect ligand-induced $\mathrm{Ca}^{2+}$ mobilization. 80

Figure 3.18. Mutation of $\mathrm{CxxC}$ and/or PDZ motif(s) of $\mathrm{LPA}_{2}-\mathrm{CT}$ attenuates ERK1/2 and Akt activation by LPA

Figure 3.19. Mutation of $\mathrm{CxxC}$ and $\mathrm{PDZ}$ motifs of $\mathrm{LPA}_{2}-\mathrm{CT}$ abolishes protection against adriamycin-induced apoptosis revealed by Annexin $\mathrm{V}$ staining 
Figure 3.20. Mutation of $\mathrm{CxxC}$ and $\mathrm{PDZ}$ motifs of $\mathrm{LPA}_{2}-\mathrm{CT}$ abolishes protection against adriamycin-induced apoptosis revealed by DNA

fragmentation assay ................................................................... 84

Figure 3.21. Mutation of $\mathrm{CxxC}$ and/or PDZ motif(s) of $\mathrm{LPA}_{2}-\mathrm{CT}$ abolishes protection against adriamycin-induced Caspase 3 activation and regulation of Siva-1 expression .85

Figure 3.22. Scheme of antiapoptotic mechanism of $\mathrm{LPA}_{2}$ by protein-protein interaction 89 


\section{LIST OF ABBREVIATIONS}

$\mathrm{ABE}$

AIF

ANT

APAF-1

AR

AT1R

ATM/ATR

BGH

$\mathrm{BH}$

Biotin-HPDP

BJAB

BrdU

BSA

CARD

CFTR

CHK

CHX

CLL

cPA

CT

DAPI

DISC

DSB

EDG

EGF

ELISA

ERK

FADD acyl-biotin exchange

apoptosis inducing factor

adenine nucleotide translocator

apoptotic protease activating factor-1

adrenergic receptor

angiotensin receptor type I

ataxia-telengiectasia mutated/ ataxiatelengiectasia and RAD3 related

bovine growth hormone

Bcl-2 homology

N-[6-(biotinamido)hexyl]-3'-(2'pyridyldithio)propionamide

Burkitt's lymphoma cell lines

bromodeoxyuridine

bovine serum albumin

caspase recruitment domain

cystic fibrosis transmembrane conductance regulator

checkpoint kinase

cycloheximide

chronic lymphocytic leukemia

cyclic glycerol-3-phosphate

carboxyl terminal

4', 6-diamidino-2-phenylindole

death-inducing signaling complex

double strand break

endothelial differential gene

epidermal growth factor

enzyme-linked immunosorbent assay

extracellular signal-regulated kinase

Fas associated death domain 


\begin{tabular}{|c|c|}
\hline GI & gastrointestinal \\
\hline GPCR & G protein coupled receptor \\
\hline Gy & Gray (radiation unit) \\
\hline $\mathrm{H} \& \mathrm{E}$ & hematoxylin and eosin \\
\hline IAPs & inhibitor of apoptosis proteins \\
\hline IEC6 & intestinal epithelial cell line-6 \\
\hline IR & irradiation \\
\hline $\mathrm{KO}$ & knockout \\
\hline $\mathrm{LD}_{100 / 30}$ & lethal dose that kills $100 \%$ of the mice in 30 days \\
\hline LIM & Lin11, Isl-1, Mec-3 \\
\hline LPA & lysophosphatidic acid \\
\hline $\mathrm{LPA}_{1-7}$ & LPA receptor $1-7$ \\
\hline LPP & lipoma preferred partner \\
\hline LPP1 & lipid phophate phosphatase 1 \\
\hline MEF & mouse embryonic fibroblast \\
\hline MEK & map-E kinase \\
\hline MOMP & mitochondria outer membrane permeabilization \\
\hline NEM & N-ethylmaleimide \\
\hline NES & nuclear exporting signal \\
\hline NHERF2 & $\mathrm{Na}^{+} / \mathrm{H}^{+}$exchanger regulatory factor- 2 \\
\hline OTP & octadecenyl thiophosphate \\
\hline $\mathrm{PA}$ & phosphatidic acid \\
\hline PATs & protein acyl transferases \\
\hline PDZ & PSD95/Dlg/ZO-1 \\
\hline PGP & plasmologen glycerophosphate \\
\hline $\mathrm{PI} 3 \mathrm{~K}$ & phosphoinositide3-kinase \\
\hline PLGF & phospholipids growth factor \\
\hline PPAR $\gamma$ & peroxisome proliferator-activated receptor $\gamma$ \\
\hline $\mathrm{pRB}$ & retinobalstoma protein \\
\hline PTP & permeability transition pore \\
\hline PTX & pertussis toxin \\
\hline RH7777 & rat hepatoma cell line \\
\hline
\end{tabular}


S1P

SAH

SHP-2

SPC

TLC

TM

TNF

TNFR

TRADD

TRAF

TRAIL

TRIP6

VDAC

ZRP-1 sphingosine-1-phosphate

Siva-1 amphipathic helical region

src homology phophatase-2

sphingosylphospharylcholine

thin-layer chromatography

transmembrane

tumor necrosis factor

TNF receptor

TNFR associated death domain

TNFR associated factor

TNF-related apoptosis-inducing ligand

Thyroid hormone receptor interacting protein-6

voltage dependent anion channel

zxyin-related protein-1 


\section{CHAPTER 1. GENERAL INTRODUCTION}

\subsection{LPA RECEPTOR AND SIGNALING}

\subsubsection{LPA Receptors and Signaling}

Lysophosphatidic acid (LPA) is a naturally occurring lipid mediator. It is a prototypical member of the biological lipid family of phospholipid growth factors (PLGFs) including phosphatidic acid (PA), plasmologen glycerophosphate (PGP), sphiongosine-1-phosphate (S1P), cyclic glycerol-3-phosphate (cPA), sphingosylphosphorylcholine (SPC) and lysophosphatidylcholine (LPC) (Tigyi, 2001 review). LPA is released by platelets, adipocytes, fibroblasts, endothelial cells, cultured postmitotic neurons, and several types of cancer cells (Aoki, 2004 review). It exists abundantly in biological fluids such as serum, saliva, follicular fluid, seminal fluid and malignant effusions and induces a vast array of biological responses affecting cell growth, survival differentiation, migration and morphology (Tigyi \& Parrill, 2003 review). It functions through activation of its cognate membrane-bound receptors (LPARs) that belong to G protein coupled receptors (GPCR) family. $\mathrm{LPA}_{1-3}$ belongs to the Endothelial Differentiation Gene (EDG) family and share $50 \%$ sequence homology with the most divergence at the C-terminal ends (Anliker \& Chun, 2004 review). GPR23/LPA 4 (Noguchi, 2003) and GPR92/LPA (Kotarsky, 2006) are more closely related to purinergic receptors than EDG receptors. GPR87 (potential LPA 6 ) and P2Y5 (potential $\mathrm{LPA}_{7}$ ), belonging to the $\mathrm{P} 2 \mathrm{Y}$ family, have recently been deorphanized and found to be LPA receptors (Shimomura, 2007; Tabata, 2007). P2Y5 has been found to be essential for maintenance of human hair growth (Pasternack, 2008; Shimomura, 2008). More interestingly, proxisome proliferators-activated receptor $\gamma(\operatorname{PPAR} \gamma)$ has been identified as an intracellular receptor of LPA (McIntyre, 2003).

Upon LPA stimulation, LPARs interact with different $G$ proteins $\left(G_{s}, G_{i / 0}, G_{q}\right.$ and $\mathrm{G}_{12 / 13}$ ) to activate various downstream effector pathways, which explains the diverse cellular effects of LPA (Mills \& Moolenaar, 2003 review; Ishii I, 2004 review). LPA displays robust coupling to $\mathrm{G}_{\mathrm{i} / \mathrm{o}}$ and $\mathrm{G}_{12 / 13}$ and weak coupling to $\mathrm{G}_{\mathrm{q}}$. While $\mathrm{LPA}_{2}$ and $\mathrm{LPA}_{3}$ interact more strongly with $\mathrm{G}_{\mathrm{q}}$. $\mathrm{LPA}_{2}$ can also couple to $\mathrm{G}_{12 / 13}$. Signaling through $\mathrm{G}_{\mathrm{q}}$ activates PLC, which subsequently hydrolyzes $\mathrm{PIP}_{2}$ and elicits $\mathrm{Ca}^{2+}$ mobilization. $\mathrm{G}_{\mathrm{i} / \mathrm{o}}$ mediates inhibition of adenylyl cyclase that decreases cAMP levels. It also mediated stimulation of Ras-MAP kinase and PI-3-kinase/Akt pathways; $\mathrm{G}_{12 / 13}$ is involved in activation of small GTPase RhoA, which regulates actomyosin-based cytoskeleton contraction. $\mathrm{G}_{\mathrm{s}}$ activates adenylyl cyclase to increase cAMP. LPA $\mathrm{LA}_{4}$ couples to $\mathrm{G}_{\mathrm{s}}$ and $\mathrm{G}_{\mathrm{q}} / \mathrm{G}_{\mathrm{i}}$ to mediate $\mathrm{Ca}^{2+}$ signaling and $\mathrm{G}_{12 / 13}$ to mediate Rho activation (Lee, 2007). LPA couples to $\mathrm{G}_{\mathrm{q}}, \mathrm{G}_{12 / 13}$ and $\mathrm{G}_{\beta \gamma}$ to activate $\mathrm{Ca}^{2+}$, Rho and increase cAMP respectively (Lee, 2006). Activation of P2Y5 increases cAMP (Pasternack, 2008)

In adult mice, $\mathrm{LPA}_{1}$ is widely expressed with high levels in testis, brain, lung, heart, spleen and intestine, and moderate levels in kidney, stomach, thymus and muscle. 
$\mathrm{LPA}_{2}$ and $\mathrm{LPA}_{3}$ are somewhat more restricted. $\mathrm{LPA}_{2}$ is generally low in most tissues except testis, protstate, pancreas and leukocytes. Expression of $\mathrm{LPA}_{3}$ is high in kidney, testis, lung and to a lesser extent in small intestine, heart, spleen, thymus and stomach (Anliker \& Chun, 2004 review). The ratio of $\mathrm{LPA}_{2} / \mathrm{LPA}_{1}$ increased markedly during malignant transformation (Shida, 2004). $\mathrm{LPA}_{4}$ has a wide expression in embryonic and restricted to heart and skin in adult tissues (Lee, 2007). LPA 5 has a broad low level of expression in many tissues with higher expression in small intestine and sensory dorsal root ganglion (Lee, 2006). $\mathrm{LPA}_{6}$ is expressed in genital organ system, brain and skeletal muscle, not in heart, lung, kidney, liver or intestine (Tabata, 2007).

$\mathrm{LPA}_{1}$ knockout mice revealed that $\mathrm{LPA}_{1}$ is required for normal development of an inborn neonatal behavior. $\mathrm{LPA}_{1}-/-$ mice resulted in $50 \%$ neonatal lethality and suckling impairment in neonatal pups. $\mathrm{LPA}_{1}-/$ - primary cortical culture revealed loss of LPA responses (Contos, 2000). In contrast, $\mathrm{LPA}_{2}-/$ - mice don't show obvious phenotypic abnormality (Contos, 2002). $\mathrm{LPA}_{3}$ knockout reveals import role of $\mathrm{LPA}_{3}$ signaling in implantation and embryo spacing (Ye, 2005).

Accumulating evidence has shown that LPA is a potent antiapoptotic factor. LPA protects various cell lines from apoptosis induced by different apoptotic stimuli: serumdeprived apoptosis in fibroblasts (Fang, 2000), macrophages (Koh, 1998), T cells (Goetzl, 2000), Schwann cells (Weiner, 1999), renal proximal tubular cells (Levine, 1997), intestinal epithelial cells (Deng 2002); chemotherapeutic agents-induced apoptosis in BJAB and I-83 B cell lines, primary chronic lymphocytic leukemia (CLL) cell lines (Hu, 2005), prostate cancer cells (Raj, 2004), intestinal epithelial cells (Deng, 2002), colon cancer cell lines (Rusovici, 2007) and ovarian cancer cell lines (Frankel 1996, Meng, 2005); Death receptor-induced apoptosis (Kang, 2004); radiation-induced apoptosis in epithelium (Deng, 2002). The apoptosis protection by LPA is mainly mediated by $\mathrm{LPA}_{1}$ and $\mathrm{LPA}_{2}$. ERK and/or Akt/PKB signaling pathways, among others, have been identified to mediate the LPA anti-apoptotic effects in most of these cell lines (Radeff-Huang, 2004).

Our group has found that LPA protects IEC-6 cells from apoptosis induced by different stimuli: serum withdrawal, DNA damaging agent (camptothecin), TNF $\alpha$ and $\gamma$ irradiation (Deng, 2002). LPA protects IEC-6 from apoptosis through inhibition of the mitochondria apoptotic pathway (Deng, 2003). This survival role of LPA doesn't involve transactivation of EGFR, ErbB2 or PDGFR (Deng, 2004).

\subsubsection{OTP: A Rationally Designed LPA Mimic}

Studies on LPA, a naturally occurring lipid with a diversity of biological function, have provided us with knowledge of LPA function. It would be practical to utilize these features of LPA to better serve the human kind. Our lab has been focusing on rational drug design using computational modeling and docking studies (Tigyi \& Parrill, 2003 review). In an effort to find LPAR subtype-specific agonists and antagonists that allow dissection of the roles of LPAR subtype functions, we have synthesized the compound 
octadecenyl thiophosphate (OTP) based on computational modeling of LPA receptor (Virag, 2003; Durgam, 2005). In structure, OTP lacking the glycerol backbone, consists of only a polar phosphate head group and a fatty acid tail. This makes OTP more resistant to the degradation by lipase and phospholipase. Also sulfur replacement of oxygen at the phosphate makes OTP a poor substrate for lipid phosphate phosphatase (LPP). However, this modification doesn't interfere with either recognition or interaction with LPARs.

These properties of OTP prompted us to explore its antiapoptotic role both in vitro and in vivo, due to its structure resemblance to LPA.

\subsection{APOPTOSIS IN SMALL INTESTINE EPITHELIUM}

Apoptosis is generally defined as the morphological end point of a complex signaling cascade resulting in the activation of intracellular proteases denoted as caspases. The process of apoptosis is the end point of an energy dependent cascade of events initiated by death inducing stimuli. Two distinct apoptotic pathways operate in mammalian cells: intrinsic and extrinsic apoptotic pathways (Fig. 1.1). The intracellular stimuli, like genotoxic stresses and radiation, trigger the mitochondrial pathway, in which release of cytochrome $\mathrm{C}$ from the mitochondria is the key event leading to caspase activation. For the extrinsic pathway, the stimuli are death ligands (TNF $\alpha$, Fas ligand, TRAIL). Apoptosis is initiated by ligand binding to death receptors (Fas, TNFR, interferon and TRAIL receptors) (Jin, 2005 review; Chowdhury, 2006 review).

\subsubsection{Intrinsic (Mitochondria-mediated) Apoptotic Pathway}

Activation of this pathway is triggered by the release of cytochrome $\mathrm{C}$ from mitochondria intermembrane space to the cytosol. The released cytochorome $\mathrm{C}$ binds APAF-1 (apoptotic protease activating factor-1) via its WD40 domain and, in the presence of ATP, binding of which induces conformational change and CARD (caspase recruitment domain) exposure of APAF-1. Usually the CARD domain of APAF-1 is bound by two of its WD40 domains and is exposed when cytochrome $\mathrm{c}$ binds to the WD40. Subsequently the procaspase 9 is recruited to the CARD forming a high molecular weight complex called apoptosome. This complex formation resulted in cleavage of procaspase 9 to active caspase 9 , which subsequently activate down stream caspases, namely caspase 3 and 7 . A protelytic cascade is then initiated with the cleavage of procaspase 2, 6, 8 and 10, leading to the dismantling of the cell (Danial \& Korsmeyer, 2004).

IAPs (inhibitor of apoptosis proteins) act as endogenous caspase inhibitors by binding to active caspase 9 and 3, controlling the activation of effector caspases downstream of apoptosome. IAPs have been reported to function as an E3-ubiquitin ligase that targets active caspase for rapid degradation (Suzuki, 2001). In turn, IAPs are regulated by its antagonists: Smac/DIABLO and Omi/HtrA2, two mitochondrial IMS proteins released upon mitochondria outer membrane permeabilization (MOMP). These 2 


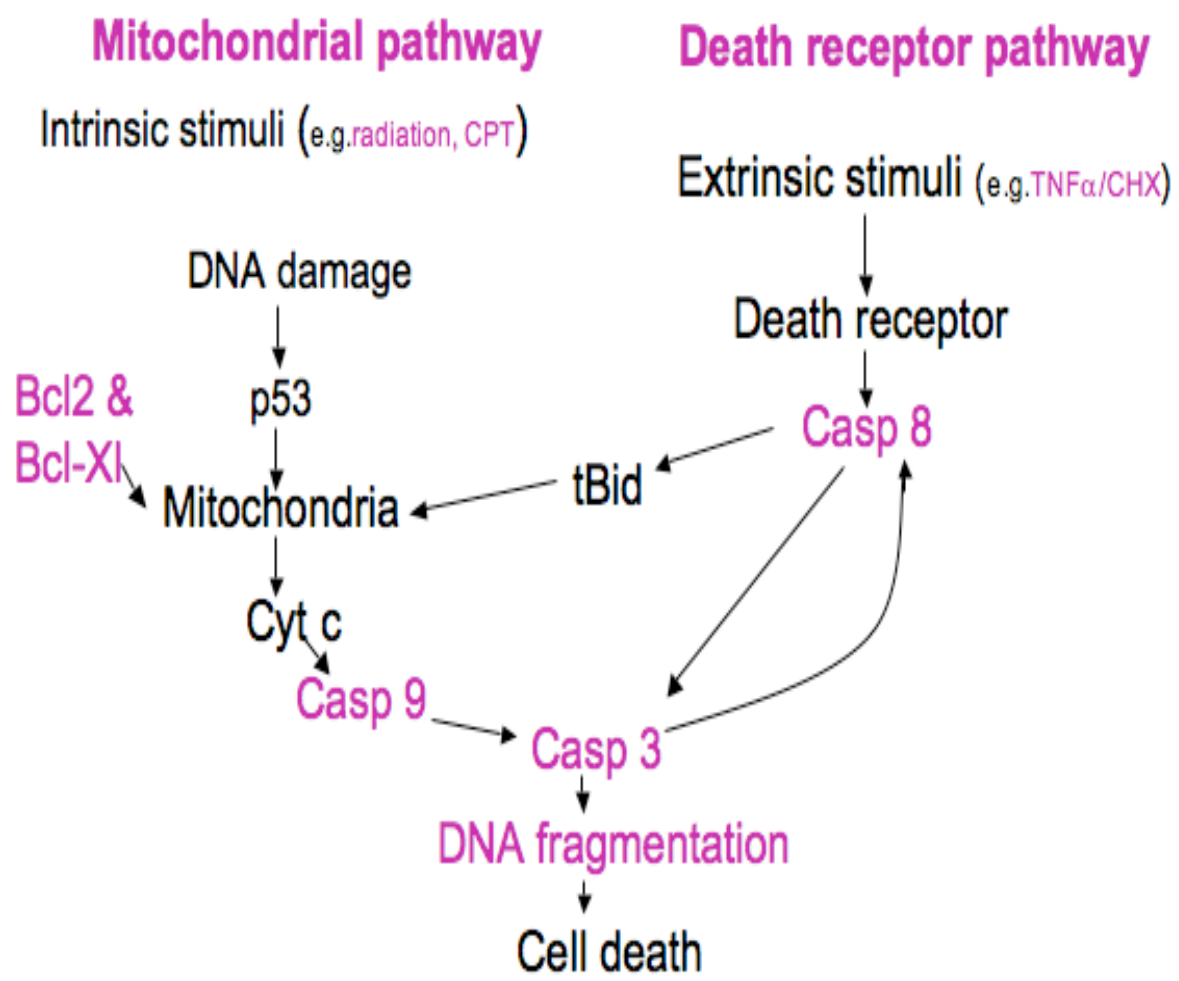

Figure 1.1. Apoptotic pathways. Schematic representation of the simplified apoptotic pathways in mammalian cells. 
proteins bind to XIAP via an AVP (I/S) sequence at their $\mathrm{N}$ termini and antagonize IAP inhibition of caspases (Spierings, 2005 review).

AIF (apoptosis inducing factor) and Endonuclease $\mathrm{G}$ are also released from mitochondria and provoke caspase-independent DNA degradation. The precise molecular mechanism by which apoptogenic proteins are released from mitochondria is still elusive. Different models have been proposed, with many implicating the MOMP. The mitochondria permeability transition pore (PTP), consisting of the ANT protein (Adenine Nucleotide Translocator), the VDAC (Voltage dependent anion channel), the benzodiazepine receptor, and cyclophilin D, is thought to play an important role (Bras, 2005 review).

Upstream of MOMP, Bcl2 family members are believed to be fundamental regulators of MOMP and subsequent release of mitochondrial factors (Spierings, 2005). Bcl2 gene was first discovered in human B cell lymphomas (Tsujimoto, 1987). Many members have been discovered and categorized into two main subfamilies: proapoptotic and antiapoptotic. They share at least one of four Bcl-2 homology (BH1-4) domains. The known antiapoptotic members include Bcl-2, Bcl-xl, mcl-1, bcl-w and A1, which show sequence conservation among all four domains. The proapoptotic group is further divided into $\mathrm{BH} 1-3$ and $\mathrm{BH} 3$-only proteins. $\mathrm{BH} 1-3$ proteins share three of the $\mathrm{BH}$ domains, including Bax, Bak and Bok. The BH3-only proteins are Bid, Bim, Bik, Bmf, Bad, Hrk, BNIP3, Noxa and PUMA. An important feature of the Bcl2 family members is the ability to form homo or heterodimers, suggesting the neutralization of competition between these proteins (Schultz, 2003 review; Shibe, 2006 review).

While it is clearly elucidated that how MOMP leads to caspase activation, the exact mechanism that how Bcl-2 family members controls the MOMP is not known. Douglas R Green proposed four models for this (Green, 2006 review). The first one is 'death by default' model involving the interplay of the three subfamilies of Bcl-2. In this model Bax and Bak are constitutively active and held in check by antiapoptotic Bcl-2 proteins. BH3-only proteins neutralize the anti apoptotic Bcl-2 proteins, release Bax and Bak that have channel forming capabilities and result in MOMP. The second model is 'life by default' model in which Bax and Bak are activated by the BH3-only proteins (Bid and Bim in this case), MOMP occurs when other BH3-only proteins neutralize antiapoptotic proteins. The third model proposes that neutralization of antiapoptotic Bcl-2 proteins leads to death only in cells that have been stressed to engage the direct activators of Bax and Bak but are kept alive by one or more of the antiapoptotic Bcl-2 family member. The fourth model is that proteins like p53 and others may function like Bid or Bim to activate Bax and Bak or Bax and Bak can be directly activated by conditions in the cell without a requirement for other proteins, like detergents and mild heat (Green, 2006 review). 


\subsubsection{Extrinsic (Death Receptor-mediated) Apoptotic Pathway}

The extrinsic apoptotic pathway is initiated upon ligation of death receptors by their cognate ligands that belong to TNF family. Death receptors belong to the TNF superfamily of the cell surface receptors. They contain a cytoplasmic protein motif termed the death domain (about 80 conserved amino acids in length) that enables death receptors to engage the cell's apoptotic machinery (Nagata, 1997). These receptors are activated upon their ligands binding. This binding results in receptor trimerization, clustering of the receptors' death domains and recruitment of adaptor molecules (e.g. Fas associated death domain [FADD] or TNFR associated death domain [TRADD]) through homophilic interaction mediated by the death domain (Chinnaiyan, 1995; Hsu, 1995). FADD and TRADD are adaptor proteins where different intermediates can bind and lead to death or survival pathway activtion. When FADD recruits pro-caspase 8 to the activated CD95 receptor to form the CD95 death-inducing signaling complex (DISC), oligomerization of pro-caspase 8 upon DISC formation drives its activation through selfcleavage. Caspase 8 activates downstream effector caspases such as caspase 3. In some cell lines, if the initial caspase activity is low, activated caspase 3 can positively feed back to caspase 8 and reinforce its activation.

\subsubsection{Physiology of Small Intestinal Stem Cells}

The mammalian intestinal epithelium provides an excellent model for the study of cell proliferation, migration and differentiation in that it represents one of the fastest renewal systems. The mouse small intestine is lined by a single layer of epithelium that consists of flask-shaped invaginations known as crypts and finger-like luminal protrusions termed villi. The epithelia maintains steady state by replenishing the loss of cells at the tip of villus with proliferating cells derived from the stem cells at the crypts through migration and differentiation. There are four differentiated cell types: enterocytes, goblet cells, enteroendocrine cells and Paneth cells. The differentiation process operates by a bidirectional migration: Paneth cells migrate to the bottom of the crypt, while the other three types of cells upward to the tip of villi.

Based on cell kinetic, lineage tracking, somatic mutation studies combined with complex mathematical modeling, Potten CS proposed a stem cell hierarchy organization in the crypt (Marshman, 2002)(Fig.1.2). This model suggests a 4-6 actual /functional stem cells per crypt in steady state. They are located at cell position $4-5$, immediately above a population of fully differentiated Paneth cells. These stem cells divide asymmetrically. They produce a daughter cell that remains at the $4^{\text {th }}$ cell position and the other daughter cell is displaced one cell position away. When this stem cell divides next time, the transit cell just above it is displaced one cell position further up the crypt. In this way, the stem cells maintain their number and at same time producing daughter cells that possess a series of cell lineage of up to six discrete cell generations. The distribution of cell lineage is related to its topographical position in the crypt. This model is really attractive for studying stem cells with the fact that there is no specific histologic or immunologic marker of the intestinal stem cells. If the actual stem cells die, they are 


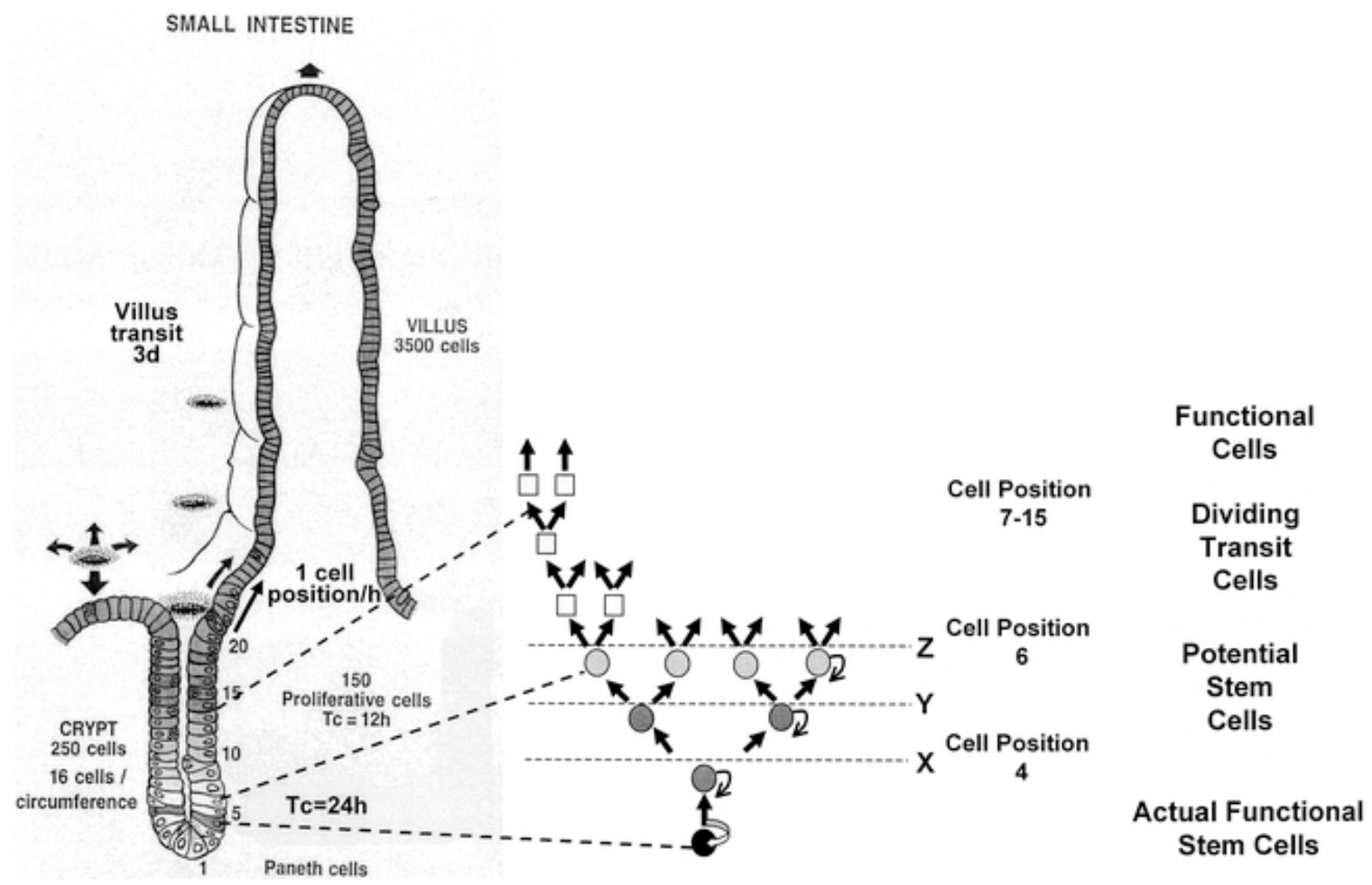

Figure 1.2. Current model for the cell lineage in small intestinal crypts. The position of a cell in the lineage can be related to its topographical position within the tissue.

Reprinted with permission from Radiation Research Society: Radiation Research. Potten CS. Radiation, the ideal cytotoxic agent for studying the cell biology of tissues such as the small intestine. Radiat Res. 2004 Feb;161(2):123-36. 
replaced by other surviving stem cells or by their immediate daughter (transit cells. The first three generations of transit cells around 30-40 in number are believed to be potential stem cells or clonogenic stem cells, while transit 4-6 generations are about 120 dividing cells (dividing transit cells). They don't have stem cell attributes.

\subsubsection{Radiobiology in Mouse Small Intestinal Stem Cells}

$\gamma$-irradiation $(\gamma \mathrm{IR})$ is one of the typical genotoxic stresses that induce DNA damage and primarily results in double strand breaks (DSBs), which activate DNA damage checkpoints to initate signals ultimately leading to binary decision between cell death and cell survival. In mammals, the signal of DNA damage caused by $\gamma$ IR is sensed by sensor proteins like RAD9, RAD1, RAD17, HUS1, transduced by ATM/ATR, which are protein kinases that phosphorylate target effector proteins such as $\mathrm{CHK} 1 / \mathrm{CHK} 2$. The well-known effector responses to DNA damage are cell growth arrest, DSB repair or apoptosis. Key to these complex cellular responses is the tumor suppressor p53 that has been recognized as an important checkpoint protein-guardian of the genome. In some systems, but not all, there is a close relationship between radiation-induced accumulation of p53, transactivation of p53 target genes and the consequent adaptive responses, including apoptosis. WT p53 is a short-lived protein with a rapid turnover under normal conditions. Upon genotoxic insult, a rapid stabilization of the $\mathrm{p} 53$ protein by posttranslational modification and its activation leads to cell cycle arrest and /or apoptosis. The best understood player in this pathway is $\mathrm{mdm} 2$. The level of $\mathrm{p} 53$ protein accumulation in response to IR primarily relates to the intensity of DNA damage (Fei \& El-Deiry, 2003 review). The dosage of IR predominately contributes to the outcomes of a cell fate. Apoptosis occurs in response to a high dose of IR when the damage is irreparable. The mechanism by which p53 mediates cell arrest and apoptosis is through both transcriptional and transcription-independent phenomena. p53 mediates cell cycle arrest in the G1/S and G2 phases of the cell cycle. In the G1/S phase, the best understood is the regulation of $\mathrm{p} 21^{\mathrm{Waf} 1 / \mathrm{Cip} 1}$. In G1, p21 negatively regulates the cyclin E/cdk2 kinase and cyclin D/cdk4-6. These kinases phosphorylate pRB and the related proteins $\mathrm{p} 107$ and p130 during G1. pRB in turn regulates the E2F mediated transcription required for cell cycle progression. When p53 is activated, p21 is activated which inhibits phosphorylation of $\mathrm{pRB}$. With hypophosphorylated pRB, E2F remains bound to pRB and E2F couldn't activate transcription, leading cell cycle arrest (Hickman, 2002 review, Harris \& Levine, 2005 review, Schuler \& Green, 2005 review).

A large number of genes regulated by p53 are known to regulate apoptosis: Bax, Noxa, Puma. By mediating these apoptotic molecules, p53 is linked to intrinsic apoptotic pathway. In addition, p53 regulates a series of genes that initiate the extrinsic apoptotic pathway (Fas ligand, killer Dr receptor), resulting activation of caspase 8. Accumulating data show that p53 has a transcription-independent proapoptotic function (Moll, 2005 review).

Overall a cell's fate of cell cycle arrest, senescence or apoptosis appears to depend on the nature of the stress signal, the type and location of the protein modification on the 
p53, those proteins associated with p53 and even the pattern of gene expression occurring in the specific tissue.

Radiation has been found to be the ideal cytotoxic agent to study the cell biology of small intestine (Potten, 2004 review). Studies using lower dose of radiation ( $<1 \mathrm{~Gy}$ ) revealed earlier apoptosis peaked at 3-6 $\mathrm{h}$ post-irradiation and there is a natural background level of apoptosis, which is termed spontaneous apoptosis and p53 independent. This spontaneous apoptosis also occurs in normal human small intestine. These apoptotic cells always occur at cell position 4-5. The dose-response relationship study of these radiation-sensitive cells revealed that that there are about 4-6 apoptosissusceptible cells per crypt located at around cell position 4-5 and these cell lack repair capacity. The conclusion was drawn from these studies: the apoptotic response may be part of the genome protection mechanism operating on the stem cells in the small intestine (Potten, 2004 review).

Increasing dose of radiation will kill the potential stem cells. Crypt survival curve drawn by counting the number of regenerating crypt-like foci at 3-4 days after different doses of radiation show that a large shoulder and a roughly exponential region from which the sensitivity of the clonogenic target cells can be determined (Fig.1.3). 8Gy will cause apoptotic body formation, depletion of crypt cells, and subsequent loss of the villi within 5-7 days, which is faster than bone marrow to show radiation effects. Higher dose would cause the complete sterilization of many crypts and consequent ulcers. Just one surviving clonogenic cell in a crypt allows its regeneration. Based on these findings a functional assay for quantifying stem cell survival following acute cytotoxic injury to the replicating cell population has been developed----Crypt Clonal Regeneration assay (the microcolony assay). This method involves counting the number of regenerating crypt-like foci between 3-4 days after different doses of radiation. These regenerating foci are easily recognized by routine $\mathrm{H} \& \mathrm{E}$ staining of paraffin-embedded sections.

\subsection{LIM AND PDZ DOMAIN PROTEINS INTERACTING WITH LPA 2 C- TERMINUS}

It is well known GPCR exerts their effects on cells through coupling to different G proteins. Recent studies reveal that GPCR also interact with cytoplasmic scaffold proteins, which link receptors to various downstream signaling molecules (Brady \& Limbird, 2002 review, Hall \& Lefkowitz, 2002 review; Luttrell, 2005 review). Scaffold proteins are defined as proteins that associate with two or more partners to enhance the efficiency and/or specificity of the cellular signaling pathways. There are several scaffold proteins have been demonstrated to interact with GPCR (Milligan \& White, 2001 review; Hall \& Lefkowitz, 2002 review).

Recent studies suggest that scaffold proteins like PDZ and LIM domain proteins interact with $\mathrm{LPA}_{2} \mathrm{C}$-terminus (CT) specifically and mediate $\mathrm{LPA}_{2}$ signaling (Oh, 2004; Wang, 2004; Xu, 2004; Yamada, 2005; Lin, 2007; Zhang, 2007). LPA 1 , LPA 2 and LPA share high homology $(>50 \%)$ in amino acid sequence except CT region, where there is 


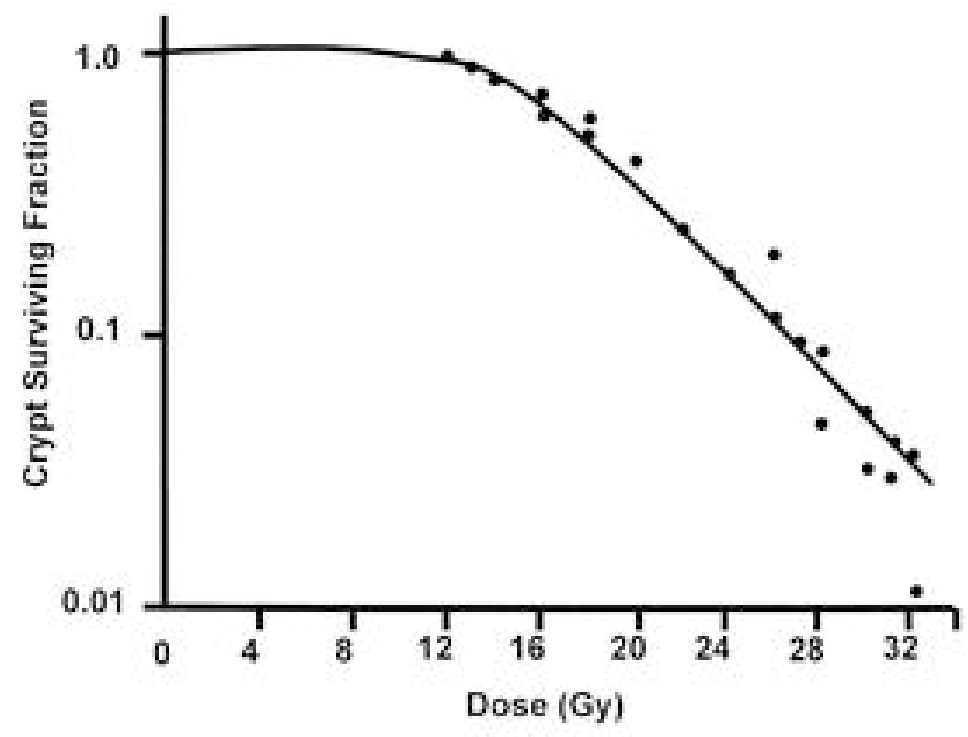

Figure 1.3. Crypt survival curve.

Reprinted with permission from Radiation Research Society. Potten CS. Radiation, the ideal cytotoxic agent for studying the cell biology of tissues such as the small intestine. Radiat Res. 2004 Feb;161(2):123-36. 
only $27 \%$ identity between the $\mathrm{LPA}_{1}$ and $\mathrm{LPA}_{2}$ receptors and $17 \%$ between the $\mathrm{LPA}_{2}$ and $\mathrm{LPA}_{3}$.

\subsubsection{PDZ Domain Proteins}

PDZ domain is a modular protein-interaction domain that consists of around 90 amino acids. It is named after the three founding PDZ containing proteins: the postsynaptic protein PSD-95/SAP90, the Drosophila septate junction protein Disc-large, and the tight junction protein $\mathrm{ZO}-1$. The PDZ domain proteins bind specifically to distinct peptide sequences in the $\mathrm{CT}$ of the target proteins, most often transmembrane receptors and channels. Based on the sequences they recognize, three classes of PDZ domain proteins have been categorized (Harris \& Lim, 2001 review). Class I PDZ domains preferentially bind to the CT motif $\mathrm{S} / \mathrm{T}-\mathrm{x}-\phi-\mathrm{COOH}$ (where $\mathrm{X}=$ any amino acid, $\phi$ $=$ hydrophobic amino acid). Class II PDZ domain proteins recognize the motif $\phi-X-\phi-$ $\mathrm{COOH}$. Class III proteins recognize $\mathrm{x}-\mathrm{x}-\mathrm{C}-\mathrm{COOH}$. There are also PDZ proteins that don't fall into these three categories. PDZ domains also mediate signaling through PDZ-PDZ interaction, recognition of internal protein sequences, and phosphatidylinositol moiety (Harris \& Lim, 2001 review; Hung \& Sheng, 2002 review; Jelen, 2003 review).

So far, at least four PDZ-containing proteins have been found to interact with $\mathrm{LPA}_{2}: \mathrm{Na}^{+} / \mathrm{H}^{+}$exchange regulatory factor 2 (NHERF2) (Oh, 2004; Yun, 2005); PDZRhoGEF (Yamada, 2005); leukemia-associated RhoGEF (Wang, 2004); membrane associated guanylate kinase-like protein with an inverted domain structure-3 (MAGI-3) (Zhang, 2007). The well-studied PDZ domain protein interacting with $\mathrm{LPA}_{2}-\mathrm{CT}$ is NHERF2. The second PDZ domain of NHERF2 interacts specifically with $\mathrm{LPA}_{2}$, but not $\mathrm{LPA}_{1}$ or $\mathrm{LPA}_{3}(\mathrm{Oh}, 2004)$. Shim's group (Yun, 2005) has found that NHERF2 regulates LPA $_{2}$-mediated mitogenic signals in Caco-2 cells. In collaboration with Dr. A.P. Naren, our group found that $\mathrm{LPA}_{2}$ receptor formed a macromolecular complex with CFTR and NHERF2 via the second PDZ domain. This macromolecular complex formation was reduced by $\mathrm{LPA}_{2}$ L351A point mutant and completely abolished by $\mathrm{LPA}_{2} \triangle \mathrm{STL}$ (Li, 2005a).

\subsubsection{LIM Domain Proteins}

The LIM domain is named by the acronym of three homeodomain proteins: Lin11, Isl-1 and Mec-3. It usually consists of approximately 55 amino acids with 8 highly conserved residues, mostly cysteine and histidine located at defined intervals. The classical LIM consensus sequence has been defined as $\mathrm{CX}_{2} \mathrm{CX}_{16-23} \mathrm{HX}_{2} \mathrm{CX}_{2} \mathrm{CX}_{2} \mathrm{CX}_{16-21}$ $\mathrm{CX}_{2}(\mathrm{C} / \mathrm{H} / \mathrm{D})$ (X denotes any amino acid) (Fig.1.4). These eight highly conserved residues are the zinc binding residues (Fig.1.4). LIM domain proteins are found in the nucleus cytoplasm or shuttle between the two compartments. These proteins have been found to play important roles in a variety of fundamental, biological processes including cytoskeletal organization, cell lineage specification and organ development as well as in disease processes like oncogenesis. These functions are mediated by interaction with 
a

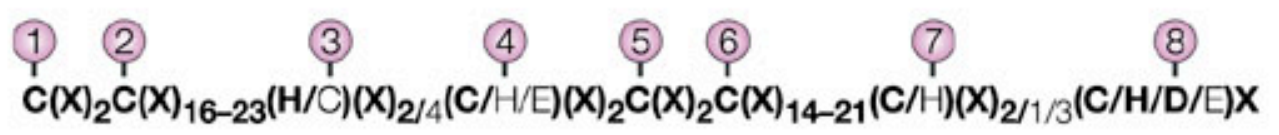

b

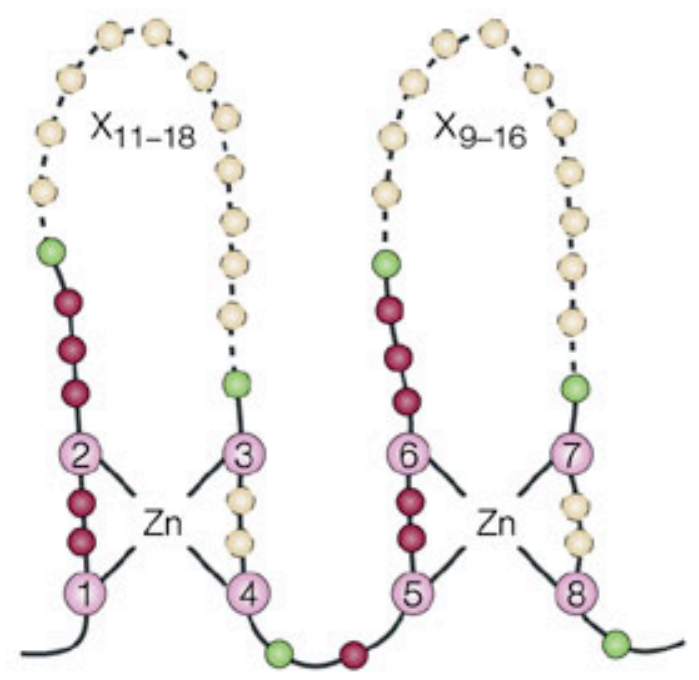

Figure 1.4. Conserved sequence and topology of the LIM domain. (A) conserved sequence of LIM domains. (B) LIM domain forms two sequencial zinc-fingers.

Reprinted with permission from Mcmillan Publishers Ltd: Nature Review Molecular Cellular Biology. Kadrmas JL, Beckerle MC. The LIM domain: from the cytoskeleton to the nucleus. Nat Rev Mol Cell Biol. 2004 Nov; 5(11): 920-31. 
proteins that have LIM domain motifs (Bach, 2000 review; Kadrmas \& Beckerle, 2004 review; Zheng, 2007).

Dr. Lin's group has found that TRIP6 (Thryoid Receptor Interacting Protein 6)/ZRP-1 (zyxin-related protein-1) interacts with $\mathrm{LPA}_{2}-\mathrm{CT}$, but not $\mathrm{LPA}_{1}$ and $\mathrm{LPA}_{3}$, using yeast two-hybrid screening and this interaction mediates LPA-dependent cell migration (Xu, 2004). TRIP6 was first identified by interaction with Thryoid-hormone receptor (Lee, 1995). It belongs to the LIM domain protein subfamily of Zyxin, which consists of Zyxin, LPP (lipoma preferred partner) and Ajuba (Macalma, 1996; Petit, 2005). These members contain a divergent N-terminal domain rich in prolines, which has a Nuclear Exporting Signal (NES) and a C-terminal region with three LIM domains (Yi \& Beckerle, 1998). In resting cells the zyxin family members localize primarily at focal adhesion plaques (Beckerle, 1997). By associating with cytoskeleton and signaling proteins, they regulate actin dynamics, cell movement, and signal transduction (Wang \& Gilmore, 2003). Also mounting evidence shows that these zyxin members shuttle between the nucleus and focal adhesion plaques. In the nucleus, these proteins interact with various transcription factors and they have been found to have transactivation ability (Wang \& Gilmore, 2003).

TRIP6 is a protein of 476 amino acids. Physiological function of TRIP6 is still elusive, though many TRIP6-interacting proteins have been identified. Besides LPA TRIP6 has been found to interact with tyrosine phosphatase PTP1E/PTP-BL (Cuppen, 2000), the V-Rel oncoprotein (Zhao, 1999), the integrin-associated adaptor protein p130 ${ }^{\text {cas }}$, the serine/threonine RIP2 (Li, 2005b), tumor suppressor protein Scrib (Petit, 2005), and a cellular energy sensor protein AMPK (Solaz-Fuster, 2006). The interaction of TRIP6 with LPA 2 promotes LPA-dependent recruitment of TRIP6 to the focal adhesion where it interacts with focal adhesion molecules: focal adhesion kinase, p130 ${ }^{\text {cas }}$, paxillin and c-Src. c-Src can phosphorylate TRIP6 at Tyr-55 and the phosphorylation mediates the LPA-induced ERK activation (Lai, 2005). Recently the same group found that PTPL1/FAP-1, which can dephosphorylate TRIP6 at Tyr-55, negatively regulates the LPA-induced morphological change and cell migration (Lai, 2007).

Interestingly to us, phosphorylation at Tyr-55 of TRIP6 by c-Src regulates LPAmediated ERK activation (Lai, 2005). Knockdown of TRIP6 drastically attenuates LPAinduced ERK1/2 activation in c-Src expressing cells (Lai, 2005). Furthermore, LPA activates Src and inhibition of Src abolishes its protective effect (Deng \& Tigyi, unpublished). TRIP6 also potentiates receptor-interacting protein 2 (RIP2)-mediated NF$\kappa \mathrm{B}$ and ERK activation ( $\mathrm{Li}, 2005 \mathrm{~b}$ ). These observations suggest that TRIP6 could be involved in mediating the anti-apoptotic effect of $\mathrm{LPA}_{2}$ by ERK activation, which is a required signal for the protective effect of LPA (Deng, 2003; E, 2007).

Siva-1 is another $\mathrm{LPA}_{2}$-interacting protein with high affinity using yeast two hybrid screens (Lin, 2007). Siva-1 is a pro-apoptotic molecule initially discovered by using the cytoplasmic tail of CD27 as bait in a yeast two-hybrid screen (Prasad, 1997). The Siva gene is organized into 4 exons, which generates two transcripts. The full-length 
transcript is called Siva-1, and the short one Siva-2 lacking the exon 2 coding sequence (Yoon, 1999). Siva-1 is not related to either death domain containing proteins like FADD or TNFR associated factors (TRAFs) domain proteins. Also, Siva-1 does not contain any Bcl-2 Homology (BH) domains. So it is not related to Bcl-2 family members. However, it contains two carboxyl zinc finger-like cysteine-rich domains and these cysteines are also present in the human and rat homologues. Overexpression of Siva-1 in various cell lines induced apoptosis (Prasad, 1997). Siva-1 expression is up-regulated during DNA damage responses (Qin, 2002; Daoud, 2003; Fortin, 2004; Jacobs 2007; Lin, 2007). More

interestingly, the Siva-1 gene is found to be a direct transcriptional target of both p53 and E2F (Fortin, 2004). Siva-1 binds to Bcl- $\mathrm{X}_{\mathrm{L}}$ and inhibits Bcl- $\mathrm{X}_{\mathrm{L}}$ mediated protection against UV radiation-induced apoptosis (Xue, 2002). A putative amphipathic helical region (SAH) is found to be sufficient to bind $\mathrm{Bcl}-\mathrm{X}_{\mathrm{L}}$ and sensitize cells to $\mathrm{UV}$ radiation induced apoptosis (Chu, 2004). The underlying mechanism involves cytochrome $\mathrm{C}$ release, activation of caspase 9 and caspase 3 . In T lymphocytes, overexpression of Siva proteins induces apoptosis via a caspase dependent mitochondrial pathway (Py, 2004). Dr. Lin's group has found that adriamycin-induced Siva-1 protein stabilization is attenuated by LPA in an $\mathrm{LPA}_{2}$-dependent manner, and the function of Siva-1 in promoting DNA damage-induced apoptosis is inhibited by LPA pretreatment. Prolonged LPA stimulation promotes the interaction, ubiquitination and degradation of Siva-1 with the $\mathrm{LPA}_{2}$ receptor. Consistent with this result, knockdown of $\mathrm{LPA}_{2}$ expression increases Siva-1 protein levels and augments adriamycin-induced caspase-3 activation and apoptosis (Lin, 2007). All these lines of evidence point to Siva-1 as proapoptotic. However, the mechanism of the apoptosis-promoting function of Siva-1 remains elusive.

The role of Siva-1 in apoptosis and the finding of Siva-1 interaction with $\mathrm{LPA}_{2}$ prompted us to investigate the antiapoptotic mechanism of $\mathrm{LPA}_{2}$. Amino acid alignment of $\mathrm{LPA}_{2}-\mathrm{CT}$ with TRIP6 and Siva-1 display highly conserved cysteine residues in $\mathrm{LPA}_{2}-$ $\mathrm{CT}$. Since zinc-finger mediates protein-protein interaction, we hypothesize that these cysteine residues may constitute the binding motif of LPA 2 to TRIP6 and/or Siva-1 (Feuerstein, 1994; Schmeichel \& Beckerle, 1994; Arber \& Caroni, 1996). 


\section{CHAPTER 2. THE LYSOPHOSPHATIDIC ACID TYPE 2 RECEPTOR IS REQUIRED FOR PROTECTING AGAINST RADIATION-INDUCED INTESTINAL INJURY*}

\subsection{INTRODUCTION}

The stem cells of the intestinal mucosa represent one of the most radiationvulnerable cell types in the mammalian body (Potten, 2004). Whereas free radical scavengers can ameliorate the central nervous system syndrome and the widely used bone marrow transplant can effectively treat the hematopoietic syndrome caused by exposure to lethal doses of radiation, effectively treating the gastrointestinal syndrome due to radiation-induced apoptosis of the intestinal stem cells is more difficult.

Lysophosphatidic acid (1-radyl-2-hydroxy-sn-glycero-3-phosphate, LPA) is a growth factor-like lipid mediator with antiapoptotic actions elicited through a set of G protein-coupled receptors (GPCR) (Tigyi \& Parrill, 2003; Moolenaar, 2004). At least five LPA GPCRs have been identified so far (Ishii, 2004). The $\mathrm{LPA}_{1}, \mathrm{LPA}_{2}$, and LPA receptors are encoded by the endothelial differentiation gene (EDG) family and share approximately $60 \%$ identity to each other (Tigyi \& Parrill, 2003; Ishii, 2004). $\mathrm{LPA}_{4}$ and $\mathrm{LPA}_{5}$ are distantly related to these LPA receptors of the EDG family, share only $20-24 \%$ amino acid identity with the EDG family, and functionally are less well characterized (Noguchi, 2003; Kotarshy, 2006; Lee, 2006). The mouse intestine predominantly expresses the $\mathrm{LPA}_{1}$ and $\mathrm{LPA}_{2}$ receptor subtypes(Li, 2005; Yun, 2005). Each individual LPA receptor has its distinct coupling pattern to $G$ proteins. $L P A_{1}$ couples to $G_{i}$ and $\mathrm{G}_{12 / 13} ; \mathrm{LPA}_{2}$ couples to $\mathrm{G}_{\mathrm{i}}, \mathrm{G}_{\mathrm{q}}$, and $\mathrm{G}_{12 / 13}$; while $\mathrm{LPA}_{3}$ couples to only $\mathrm{G}_{\mathrm{i}}$ and $\mathrm{G}_{\mathrm{q}}$, but not $\mathrm{G}_{12 / 13}$ (Ishii, 2000). These $\mathrm{G}$ proteins share many downstream signaling pathways that act in a cooperative manner (Yart, 2002). The activation of the phosphoinositide-3-kinase (PI3K)-AKT and MEK-ERK1/2 prosurvival pathways has been well delineated in mediating LPA-initiated antiapoptotic activity (Deng, 2002; Ye, 2002; Deng, 2003). LPA activates AKT, which in turn phosphorylates BAD and procaspase 9 leading to inhibition of apoptosis (Kang, 2004). LPA-induced activation of MEK-ERK1/2 signaling can also activate BAD phosphorylation and attenuate caspase 9 (Fang, 1999). LPA has been shown to activate NFKB, which regulates important prosurvival genes (Fang, 1999; Chou, 2005). A specific cellular response, including the antiapoptotic effect of LPA, might be mediated through a single LPA receptor or a combination of multiple receptors subtypes, which appear to be co-expressed in most cell types (Fischer, 1998). Because LPA receptors are ubiquitously expressed and because of the overlapping coupling

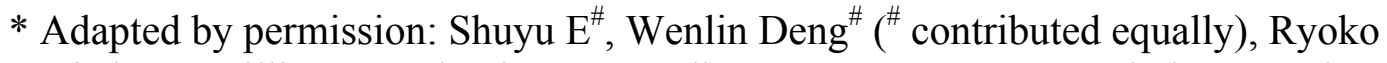
Tsukahara, William J. Valentine, Gangadhar Durgam, Veeresa Gududuru, Louisa Balazs, Venkatraman Manickam, Marcello Arsura, Lester VanMiddlesworth, Leonard R. Johnson, Abby L. Parrill, Duane D. Miller, and Gabor Tigyi. The Lysophosphatidic Acid Type 2 Receptor is Required for Protection Against Radiation-Induced Intestinal Injury. Gastroenterology 2007;132:1834-1851. 
patterns to $\mathrm{G}$ proteins, elucidating the biological responses mediated by each individual receptor requires targeted gene knockout animal models. $\mathrm{LPA}_{1}, \mathrm{LPA}_{2}$, and $\mathrm{LPA}_{1} / \mathrm{LPA}_{2}$ double knockout $(\mathrm{KO})$ animals have been generated and show minimal phenotypes (Contos, 2000; Contos, 2002; Ye, 2005). At the present time, it remains unknown which LPA receptor(s) is required for its antiapoptotic effect in the different organs.

LPA in the GI tract comes from foods such as soybean (Chevreau, 1995; FunkArchuleta, 1997; Logvinova, 1999), metabolism of phospholipids by phospholipases (Sturm, 2002), or activated platelets under pathological conditions (Sano, 2002). High levels of phospholipids have been detected in the colonic mucosa of patients with inflammatory bowel disease, and LPA significantly reduces the degree of inflammation and necrosis in a rat model of colitis (Sano, 2002). LPA has been shown to stimulate restitution of intestinal epithelia via pertussis toxin (PTX)-sensitive mechanisms (Hines, 2000). LPA $_{2}$ receptors play an important attenuating role in bacterial toxin-induced secretory diarrhea via PDZ-domain-mediated protein-protein interactions inhibiting the activation of the CFTR Cl${ }^{-}$channel (Li, 2005a). Therefore, the food-derived LPA in the lumen and its receptors on the epithelium suggest a physiological role for LPA in maintaining GI integrity that can be explored for therapeutic intervention.

Apoptosis in the intestinal epithelium is the primary pathological factor that leads to chemotherapy- or radiation-induced gastrointestinal damage (Hall, 1994; Pritchard, 1996; Potten, 2004). We formulated a hypothesis that LPA can be used for the protection of the mucosa from iatrogenic traumas - radiation injury in particular-and, if so, it could serve as a template for prosurvival drugs against radiation injury. This hypothesis is based on several lines of our work reported over the last few years. We demonstrated that LPA protected intestinal epithelia against irradiation-induced apoptosis both in vitro and in vivo (Deng, 2002), also showed that LPA achieved its antiapoptotic effects through the PTX-sensitive activation of the MEK-ERK1/2 and PI3K-AKT prosurvival pathways (Deng, 2003), and established that the antiapoptotic effect, unlike the mitogenic and motogenic effects of LPA, did not require the transregulation of several tyrosine kinase receptors (Deng, 2004). However, LPA is not an optimal drug candidate. Exogenous LPA is rapidly metabolized in the GI tract. Because complex lipids, including phospholipids, are broken down to nonpolar intermediates that traverse the plasma membrane, LPA's action is terminated by phospholipase- and lipase-mediated deacylation or (lipid) phosphatase-mediated dephosphorylation. While this mechanism rapidly renders LPA inactive, it also limits LPA's effect to the receptors present on the luminal surface of the epithelium. LPA consists of a glycerol backbone with a hydroxyl group, a phosphate group, and a fatty acid or fatty alcohol chain. We have developed and experimentally validated computational models of the EDG family of LPA receptors (Parrill, 2000; Wang, 2001; Sardar, 2002; Parrill, 2004; Fujiwara, 2005; Inagaki, 2005; Jo, 2005), and established the absolute requirement for a negatively charged headgroup and the aliphatic tail, but not for the glycerol backbone (Sardar, 2002; Virag, 2003; Durgam, 2005). Therefore, we hypothesized that long chain fatty alcohol thiophosphates mimic LPA at the EDG family receptors and at the same time are metabolically stabilized against phospholipase cleavage, which requires a glycerol backbone. Furthermore, thiophosphates tend to be poor substrates of lipid phosphatases, rendering them resistant 
to breakdown. These observations led us to explore the pharmacological and biological properties of octadecenyl thiophosphate (OTP) as an orally bioavailable, metabolically stabilized, nonabsorbing LPA mimic with radioprotective action in the gut.

The present study set multiple objectives. First, we explored the pharmacological properties of OTP by using computational and pharmacological approaches and determined its resistance to lipase and lipid phosphatase cleavage. Next, we compared the antiapoptotic effect of OTP to that of LPA in IEC-6 cells in vitro. Third, using a receptor add-back we examined which LPA receptor subtypes mediate survival signals to prevent radiation-induced apoptosis. Fourth, we examined whether orally applied OTP reduces radiation-induced apoptosis and caspase 3 activation and increases crypt survival in clonogenic assays conducted in $\mathrm{C} 57 \mathrm{BL} / 6$ mice exposed to $15 \mathrm{~Gy} \gamma$-irradiation. We also evaluated the OTP- and LPA-induced activation of those prosurvival signaling pathways in vivo that we had previously established in vitro. Finally, we tested whether intraperitoneal administration of OTP prevents death caused by $\mathrm{LD}_{100 / 30} \gamma$-irradiation. We found that OTP mitigated radiation-induced death, protected intestinal epithelial cells from apoptosis in vitro and in vivo, and was significantly more effective compared to LPA. Both LPA and OTP reduced apoptosis and caspase 3 activation and increased crypt survival in wild type and $\mathrm{LPA}_{1}$ knockout mice; however, both were ineffective in $\mathrm{LPA}_{2}$ knockout mice. Together, these data suggest that OTP is a highly effective intestinal radioprotective agent that targets $\mathrm{LPA}_{2}$ as a prosurvival receptor.

\subsection{MATERIALS AND METHODS}

\subsubsection{Reagents}

LPA18:1 (oleoyl) was purchased from Avanti Polar Lipids (Alabaster, AL). LPA and OTP (synthesized as described in (Durgam, 2005) were applied to cells complexed with fatty acid-free BSA (Sigma, St. Louis, MO) as previously described (Virag, 2003). Camptothecin and cycloheximide (CHX) were purchased from Sigma. Recombinant rat TNF- $\alpha$ was purchased from BD Pharmingen (San Diego, CA). PD98059 and PD158780 were purchased from Calbiochem (San Diego, CA). Pertussis toxin (PTX), AG1296, AG1487, Ac-DEVD-pNA and Ac-LEHD-pNA colorimetric and Ac-IETD-AFC fluorescent caspase substrates were from Biomol Laboratories Inc., (Plymouth Meeting, PA). The following antibodies and sources were used: rabbit anticaspase 3 (Santa Cruz Biotechnology, Inc., Santa Cruz, CA), rabbit antiactive caspase 3 (Abcam, Inc., Cambridge, MA), mouse monoclonal anti-JNK1 (BD Pharmingen), and mouse monoclonal anti-phospho ( $\left.\mathrm{Thr}^{183} / \mathrm{Tyr}^{185}\right)$-JNK; rabbit anti-ERK1/2 and rabbit antiphospho-( $\left.\mathrm{Tyr}^{202} / \mathrm{Tyr}^{204}\right)$ ERK1/2, rabbit anti-AKT, and rabbit anti-phospho-(Ser $\left.{ }^{473}\right)$ -

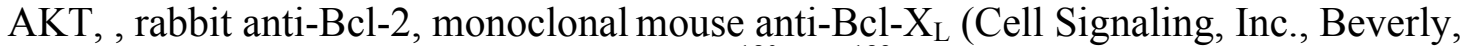
MA), monoclonal mouse anti-phospho-( $\mathrm{Thr}^{180} / \mathrm{Tyr}^{182}$ )-P38 (Promega, Madison, WI), and mouse monoclonal anti-actin (Calbiochem). Horseradish peroxidase-conjugated antirabbit and anti-mouse secondary antibody used for Western blotting was purchased from Sigma. FITC-labeled goat anti-rabbit IgG was purchased from Molecular Probe (Eugene, 
OR). Normal goat serum and VECTASHIELD Mounting Medium with DAPI were purchased from Vector Laboratories, Inc. (Burlingame, CA).

\subsubsection{Computational Modeling}

The detailed methods used to develop computational models of $\mathrm{LPA}_{1}, \mathrm{LPA}_{2}$, and $\mathrm{LPA}_{3}$ have been published previously (Wang, 2001; Sardar, 2002; Fujiwara, 2005). Briefly, our validated model of the $\mathrm{S}_{1} \mathrm{P}_{1}$ receptor (Parrill, 2000; Wang, 2001; Inagaki, 2005) was used as a template for generating LPA receptor models. Homology model development was performed using the automated algorithm implemented in the MOE software program (MOE, 2002). The best model was geometry optimized using the MMFF94 (Halgren, 1996) forcefield to a root mean square gradient of $0.1 \mathrm{kcal} / \mathrm{mol} \cdot \AA$. The individual receptor models were used in docking studies with OTP bearing a total charge of -2. Docking calculations were performed using Autodock 3.0 software (Morris, 1998) with default values for all parameters except the number of runs (Ishii, 2000), energy evaluations $\left(9.0 \times 10^{10}\right)$, generations $(60,000)$, and local search iterations $(3000)$. The complex chosen as the best geometry from each docking calculation was that with the lowest final docked energy value. Results are described using a numbering system that facilitates comparisons among homologous positions in $\mathrm{G}$ protein-coupled receptors as described by Ballesteros and Weinstein (Ballesteros, 1995). In this system, each amino acid in the transmembrane domain is given a number in the format $\mathrm{X}$.YY where $\mathrm{X}$ indicates the number of the helix where the amino acid is found and YY indicates the position relative to the most conserved amino acid in that helix at reference position 50 .

\subsubsection{Radiolabeling of $\mathrm{OTP}$}

Tritiated OTP was synthesized in our laboratory as shown in Fig. 2.1D. Pyridinium chlorochromate-mediated oxidation of oleyl alcohol gave oleyl aldehyde, which was subjected to reduction with $\mathrm{NaB}_{3} \mathrm{H}_{4}$ to form the tritiated oleyl alcohol. Alcohol 3 was phosphorylated as reported earlier (Durgam, 2005) to give protected oleyl thiophosphate ester. Treatment of 4 with methanolic $\mathrm{KOH}$ followed by acidification yielded $\left[{ }^{3} \mathrm{H}\right]-\mathrm{OTP}$. The specific activity of the product was $10.8 \mathrm{mCi} / \mathrm{mmol}$.

\subsubsection{Determining OTP Absorption from the Gut}

To determine oral absorption of OTP, female 8- to 10-week-old C57BL/6 mice (average body weight $20 \mathrm{~g}$ ) were used. Mice were maintained on a 12:12-h light-dark cycle and fed standard laboratory mouse chow and water ad libitum. Conscious mice were administered through oral gavage $1.5 \mathrm{mg} / \mathrm{kg}$ OTP in $1 \mathrm{mM}$ BSA in PBS including $10^{6} \mathrm{dpm}$ OTP. Groups of four mice were sacrificed after $30 \mathrm{~min}, 90 \mathrm{~min}$, and $180 \mathrm{~min}$, and blood samples were collected through cardiac puncture using 0.2\% EDTA anticoagulant. Ten microliters whole-blood samples from each mouse were mixed with 
A<smiles>CC[C@H](O)COC(=O)[In](O)COP(=O)(O)O</smiles>

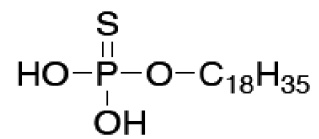

LPA 18:1

OTP
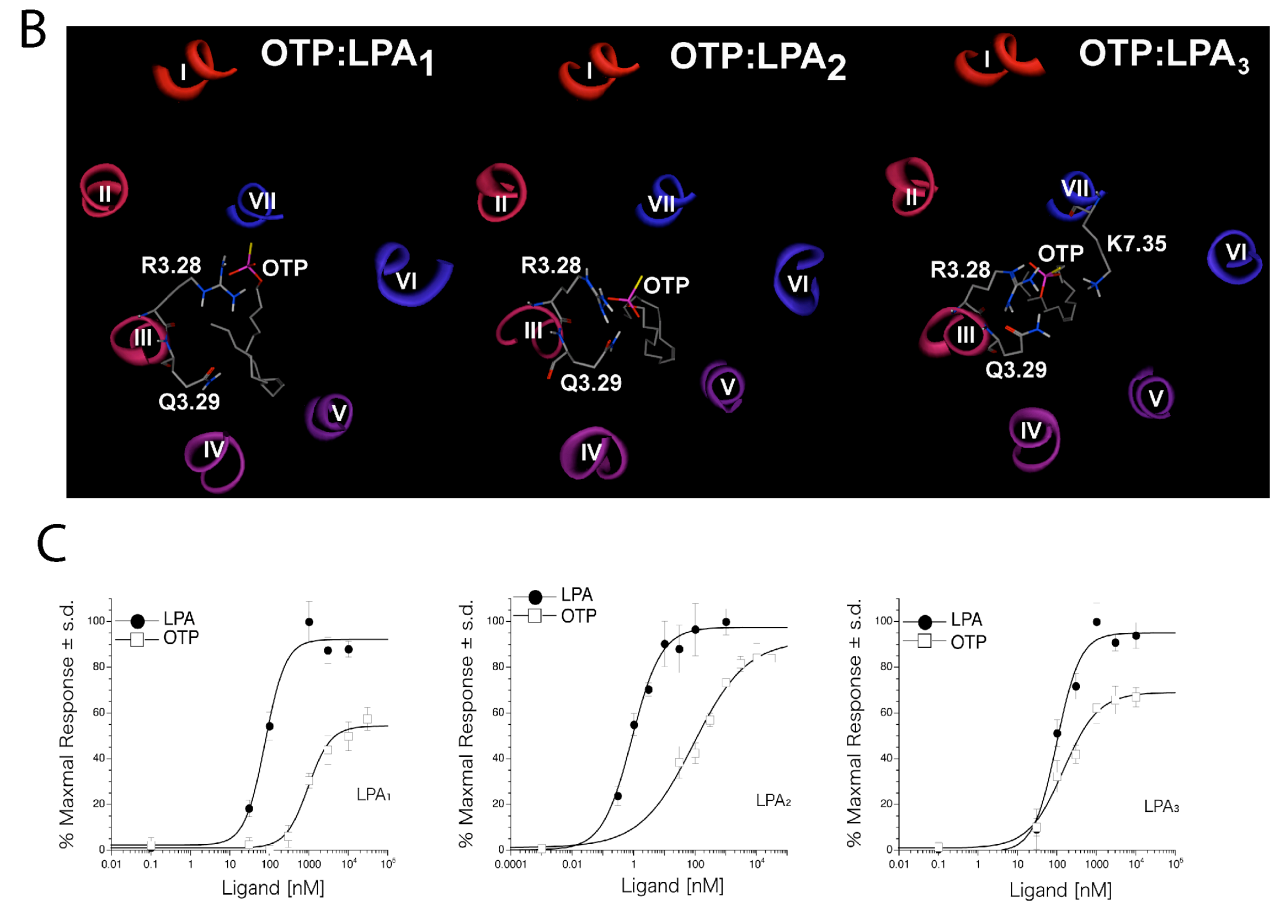

$\mathrm{D}$

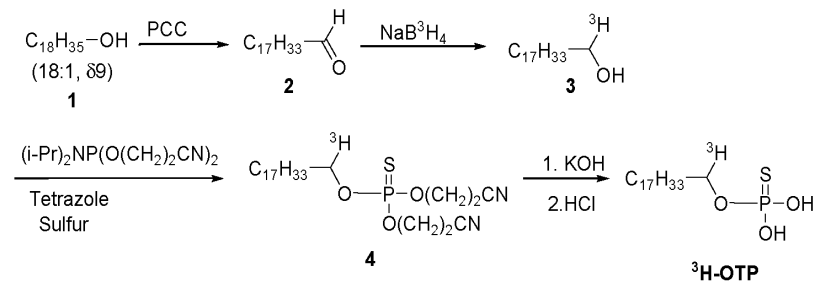

Figure 2.1. Structure, computational modeling and synthesis of OTP. Chemical structures of LPA and OTP (Panel A). Molecular models of OTP docked into the ligand binding pocket of LPA receptors (Panel B). $\mathrm{Ca}^{2+}$ transients elicited by OTP and LPA in RH7777 cells stably expressing the individual EDG family LPA receptors (Panel C). Wild type RH7777 cells show no $\mathrm{Ca}^{2+}$ transients in response to LPA up to concentrations as high as $10 \mu \mathrm{M}$ (data not shown). Synthesis of ${ }^{3} \mathrm{H}$-labeled OTP (Panel D, see 2.2 Materials and Methods for detail). 
Ecolume (Packard, Boston, MA) liquid scintillation cocktail and counted after $24 \mathrm{~h}$ of equilibration in a liquid scintillation counter.

\subsubsection{Assaying OTP Metabolism by Pancreatic Lipase and Lipid Phosphate Phosphatase 1 (LPP1)}

To determine the enzymatic stability of OTP by phospholipases, $\left[{ }^{3} \mathrm{H}\right]$-OTP $(5.2 \mathrm{x}$ $10^{6} \mathrm{dpm}$ ) mixed with $0.03 \mathrm{mM}$ cold OTP was subjected to enzymatic hydrolysis for $24 \mathrm{~h}$ by using bovine pancreatic lipase (Sigma) followed by thin-layer chromatography (TLC) separation of the products using a previously established protocol (Kates, 1988). Lipid phosphate phosphatase was obtained from mouse embryonic fibroblasts derived from mice with transgenic overexpression of the enzyme (Yue, 2004). Membrane fractions $\left(300 \mu \mathrm{g} /\right.$ reaction) prepared by centrifugation at $10^{4} \mathrm{x}$ g from transgenic LPP1 fibroblasts were added to $\left[{ }^{3} \mathrm{H}\right]$-OTP $\left(1.5 \times 10^{6} \mathrm{dpm}\right)$ mixed with $8 \mu$ mole cold OTP was subjected to LPP-1 hydrolysis for $8 \mathrm{~h}$ using a previously established protocol (Yue, 2004)). The reaction mixture was dried in vacuo and the residue was acidified with $100 \mu 11 \mathrm{~N} \mathrm{HCl}$ and extracted four times with $0.5 \mathrm{ml}$ ethyl acetate. The extracts were combined and the solvent was evaporated. The extracted reaction products were taken up in $2 \mathrm{ml}$ ethyl acetate and a $20 \mu \mathrm{l}$ aliquot was applied to TLC using methanol: ether solvent $(2: 98$. V/V) as described (Kates, 1988).

\subsubsection{Cell Culture and Induction of Apoptosis In Vitro}

IEC-6 and the rat hepatoma RH7777 cells were obtained from the American Type Culture Collection (Manassas, VA). IEC-6 cells were grown in Dulbecco's modified Eagle's medium supplemented with $10 \%$ fetal bovine serum, insulin $(10 \mu \mathrm{g} / \mathrm{ml})$, and gentamicin sulfate $(50 \mu \mathrm{g} / \mathrm{ml})$ at $37^{\circ} \mathrm{C}$ in a humidified $90 \%$ air $/ 10 \% \mathrm{CO} 2$ atmosphere. RH7777 cells, stably expressing LPA2 receptors, were provided by Dr. Fumikazu Okajima (Gunma University, Japan). RH7777 cells stably expressing LPA1 or LPA3 receptors were generated by our group and characterized elsewhere (Fischer, 2001). Wild type and stably transfected RH7777 cells were grown in Dulbecco's modified Eagle's medium with $10 \%$ fetal bovine serum and $2 \mathrm{mM}$ glutamine containing $250 \mu \mathrm{g} / \mathrm{ml} \mathrm{G} 418$ for the stable transfectants. Apoptosis in IEC-6 cells was induced by exposing them to 20 $\mu \mathrm{M}$ camptothecin or a $25-\mathrm{Gy}$ Cs 137 source $\gamma$-irradiation (Mark I model 25 Gamma Irradiator, J. L. Shepherd \& Associates, San Fernando, CA) at a rate of $4.80 \mathrm{~Gy} / \mathrm{min}$. DNA fragmentation and caspase 3 activity were measured $6 \mathrm{~h}$ after camptothecin treatment or $18 \mathrm{~h}$ post-irradiation. Apoptosis in RH7777 cells was induced by $20 \mathrm{ng} / \mathrm{ml}$ TNF- $\alpha$ plus $10 \mu \mathrm{g} / \mathrm{ml} \mathrm{CHX}$ and evaluated $6 \mathrm{~h}$ later.

\subsubsection{Pharmacological Characterization of OTP}

The ligand properties of OTP were evaluated using RH7777 cells stably transfected with each LPA receptor of the EDG family exactly as described in our 
previous report (Durgam, 2005). RH7777 cells lack endogenous $\mathrm{Ca}^{2+}$ responses to LPA applied as high as $30 \mu \mathrm{M}$, the highest concentration tested but acquire these responses upon transfection of any of the LPA receptors (Fischer, 2001; Virag, 2003; Durgam, 2005;). Briefly, RH7777 cells stably expressing human LPA1, LPA2, or LPA 3 were loaded with Fura-2 AM (Invitrogen). Changes in intracellular $\mathrm{Ca}^{2+}$ concentration were monitored by measuring the ratio of emitted light intensity at $520 \mathrm{~nm}$ in response to excitation by $340 \mathrm{~nm}$ and $380 \mathrm{~nm}$ wavelength lights, respectively using a FlexStation II (Molecular Devices, Sunnyvale, CA) robotic fluorescence plate reader. Responses were monitored for 70 seconds. $\mathrm{Ca}^{2+}$ transients were quantified automatically by calculating the difference between maximum and baseline ratio values for each sample run in triplicate.

\subsubsection{RT-PCR}

RNA was extracted using Trizol ${ }^{\circledR}$ (Invitrogen, Carlsbad, CA) from wild type and $\mathrm{KO}$ mice using four $0.5-\mathrm{cm}$ segments spaced equally along the jejunum. The following gene-specific primers were used: LPA ${ }_{1}$, forward, 5'- TCTGAAGACTGTG GTCATTGTGC-3', reverse, 5'-GCCATTAGGGTTCTCGTTGC-3', LPA2, forward, 5' CACTCCTGGCACT GCCTCTGTG-3', reverse, 5'-TAC GGC GCA TCT CAG CGT CTC G-3', $\mathrm{LPA}_{3}$, forward, 5' - ACACATGTCAATCATGAGGAT-3', reverse, 5'-GAA GACGGTGACTGTCTTAGG -3', $\mathrm{LPA}_{4}$, forward, 5'-GAAGGCTTCTCCAAACGT GTCTG-3', reverse, 5'-C CTTG TGCCTTGCAACTCTGAA-3', LPA5, forward, 5'CTGATGCTC ATCAACGTGGACC-3', reverse, 5'-TAGGGCACGAAGCACAGCAG3', $\beta$-actin, forward, 5'-GACAACG GCTCCGGCATGTG-3', reverse, 5'-TTGAGACCT TCAACACCCCAGCA -3'. Reverse-transcription and PCR was done using the Superscript III kit (Invitrogen) and a total of 31 cycles were performed; the products were applied in full to agarose gels and stained with ethidium bromide.

\subsubsection{Evaluating Apoptosis by DNA Fragmentation and Caspase Activity}

DNA fragmentation was measured by ELISA following the procedure provided with the Cell Death Detection kit from Roche, Inc. (Indianapolis, IN) as described previously (Deng, $2002 \& 2003$ ) and was expressed as absorbance units (at $405 \mathrm{~nm}$ ) per microgram protein per minute. Caspase 3 and caspase 9 activity in IEC-6 cells was measured by ELISA by using the specific Ac-DEVD-pNA chromogenic substrate (for caspase 3) and Ac-LEHD-pNA for caspase 9 as described previously ((Deng, 2002 \& 2003). Small intestine samples were washed thoroughly with PBS, scraped off the muscle layer, mixed with lysis buffer, homogenized, and centrifuged. The supernatants were collected for evaluating caspase 3 activity as described above. Caspase 3 and 9 activity was expressed as picomole pNA cleaved per minute per microgram protein. Caspase 8 activity was measured by using the specific Ac-IETD-AFC fluorescent substrate. Each reaction contained $20 \mu \mathrm{l}$ cytosolic proteins, $70 \mu \mathrm{l}$ assay buffer $(50 \mathrm{mM}$ HEPES, $\mathrm{pH}$ 7.4), $100 \mathrm{mM} \mathrm{NaCl}, 10 \mathrm{mM}$ DTT, and $1 \mathrm{mM}$ EDTA and 0.1\% (V/V) CHAPS detergent), and $10 \mu \mathrm{l}$ of $2 \mathrm{mM}$ Ac-IETD-AFC dissolved in assay buffer. The enzymatic reaction was 
carried out in 96 -well plates at $37^{\circ} \mathrm{C}$ and monitored using a FlexStation II microplate reader (Molecular Devices) at $37^{\circ} \mathrm{C}$ with fluorescence excitation at $405-\mathrm{nm}$ and emission at $505-\mathrm{nm}$. Protein concentration was measured by using the Bradford protein assay kit (Bio-Rad, Hercules, CA), and caspase 8 activity was expressed as arbitrary fluorescence units $(\mathrm{AFU}) / \mathrm{mg} / \mathrm{min}$.

\subsubsection{Western Blotting}

Caspase 3 was measured in IEC-6 cells or intestinal epithelial lysates prepared for caspase 3 activity as described in our previous reports (Deng, 2002 \& 2003). ERK1/2 and AKT were measured in total cellular protein lysates by using a previously described procedure (Deng, 2003). Briefly, IEC-6 cells were lysed in $62.5 \mathrm{mM}$ TRIS-HCl (pH 6.8), $2 \%$ SDS, $25 \%$ glycerol, $1 \mathrm{mM} \mathrm{NaF}, 1 \mathrm{mM}$ orthovanadate, and protease inhibitor cocktail (Sigma). The lysates were cleared by centrifugation $\left(10^{6} \mathrm{xg}\right.$ ) for $15 \mathrm{~min}$ at $4^{\circ} \mathrm{C}$, and supernatants were collected. Protein concentrations were determined using the BCA reagent kit (Pierce Biotechnology, Inc., Rockford, IL). For ERK1/2 $10 \mu \mathrm{g}$, for AKT 40 $\mu \mathrm{g}$, for p38 $20 \mu \mathrm{g}$, and for JNK $50 \mu \mathrm{g}$ of the cell lysate was fractionated by SDS-PAGE and transferred to PVDF membranes, blocked with 5\% nonfat milk, and incubated with various primary antibodies. Blots reacted with the appropriate HRP-conjugated secondary antibodies and developed using the SuperSignal chemiluminescence reagent (Pierce Biotechnology, Inc.).

For Western blotting of Bcl- $\mathrm{X}_{\mathrm{L}}$ and Bcl-2, IEC-6 cell were harvested and washed with PBS twice, then resuspended with 100-200 $\mu 1$ ice-cold lysis buffer (20 mM TRIS (pH 7.5), $150 \mathrm{mM} \mathrm{NaCl}, 1 \mathrm{mM}$ EDTA, $1 \mathrm{mM}$ EGTA, $1 \%$ Triton, protease inhibitor and phosphatase inhibitor cocktails (Sigma)). Cells were sonicated three times for three seconds each and the lysate was centrifuged at $16,100 \mathrm{xg}$ for $20 \mathrm{~min}$ at $4^{\circ} \mathrm{C}$. The supernatants were collected. Protein concentration was measured by using the BCA protein assay kit (Pierce).

\subsubsection{Animal Treatment and Irradiation}

The whole-body irradiation protocol was reviewed and approved by the University of Tennessee Health Science Center Animal Care and Use Committee. Eight to 10-week-old C57BL/6 mice were purchased from Harlan (Indianapolis, IN) and maintained on a 12:12-h light-dark cycle and fed standard laboratory mouse chow and water ad libitum. $\mathrm{LPA}_{1}$ and $\mathrm{LPA}_{2} \mathrm{KO}$ mice generously provided by Dr. Jerold Chun (Scripps Institute, La Jolla, CA) were bred in house and used between the ages of 8 and 12 weeks. Mice were fasted overnight before whole-body $\gamma$-irradiation $\left(\mathrm{Cs}^{137}\right.$ source at a rate of $4.80 \mathrm{~Gy} / \mathrm{min}$ ). LPA or OTP was administered by oral gavage $2 \mathrm{~h}$ before irradiation. Mice were killed either $4 \mathrm{~h}$ after irradiation by isoflurane inhalation for analysis of apoptosis or 4 days later for the clonogenic assay. Four segments of the jejunum and the ileum were fixed in 10\% neutralized formaldehyde ( $\mathrm{pH} 7.4)$ buffer and processed for histological evaluation. Mice used in the clonogenic regeneration assay 
received bromo-deoxyuridine (BrdU, $120 \mathrm{mg} / \mathrm{kg}$ ) and 5-fluoro-2'-deoxyuridine (12 $\mathrm{mg} / \mathrm{kg}$ ) intraperitoneally $2 \mathrm{~h}$ before death to label the S-phase regenerating cells in intestine.

\subsubsection{Testing the Acute Toxicology of OTP}

Female eight-week-old C57BL/6 mice (average body weight 20 g) were maintained on a 12:12 h light-dark cycle and fed standard laboratory mouse chow and water ad libitum. Mice were randomly grouped into groups of 5 and weighed daily before oral gavage of 3,10, and $30 \mathrm{mg} / \mathrm{kg}$ OTP dissolved in $1 \mathrm{mM}$ BSA in PBS. Mice were observed for an hour immediately after OTP treatment and any abnormal behavior and change in regular motor activity was recorded. Weights were monitored for five consecutive days after OTP treatment.

\subsubsection{Assessing Apoptosis and Crypt Survival in Intestine}

Paraffin cross-sections were cut perpendicular to the long axis of the small intestine and stained with hematoxylin and eosin (H\&E) or immunostained with a monoclonal anti-BrdU antibody. Epithelial cell apoptosis and crypt survival were analyzed in four sections per segment as previously described (Deng, 2002). To detect apoptosis, a minimum of 100 half crypt-villus units from each experimental group was scored. For the clonogenic assay, the number of surviving crypts per jejunal circumference was counted in the sections from the different segments. A surviving crypt was defined as a regenerative crypt that contained a cluster of 10 or more H\&E-stained cells. The viability of surviving crypts was confirmed by positive immunostaining for BrdU of incorporation into five or more crypt cells.

\subsubsection{Immunohistological Staining}

To identify the BrdU-labeled S-phase cells in the crypts, we used a staining protocol described in our previous paper (Balazs, 2001). For activated caspase 3 staining, formalin-fixed paraffin-embedded tissue slides were used with heat-mediated antigen retrieval for $15 \mathrm{~min}$. After three 5-min washes in TRIS-buffered saline (TBS, $25 \mathrm{mM}$ TRIS, $150 \mathrm{mM} \mathrm{NaCl} \mathrm{pH}$ 7.6), slides were blocked with $10 \%$ normal goat serum in TBS $/ 0.1 \%$ Tween for $2 \mathrm{~h}$ at room temperature. Rabbit active caspase 3 antibody was diluted 1:50 in $1 \% \mathrm{BSA} / \mathrm{TBS} / 0.1 \%$ Tween and incubated at $4^{\circ} \mathrm{C}$ overnight. FITC-labeled goat anti-rabbit IgG was applied for $2 \mathrm{~h}$ after wash, the slides were mounted with VECTASHIELD with DAPI (Vector Laboratories) and visualized by Nikon Eclipse 80i fluorescence microscope. At least 100 villi per animal were counted for the presence of activated caspase 3 positive cells.

Immunohistochemical staining for Bcl- $\mathrm{X}_{\mathrm{L}}$ and $\mathrm{Bcl}-2$ was done using 5- $\mu \mathrm{m}$ thick sections cut from formalin-fixed, paraffin-embedded blocks of jejunum and ileum taken 4 
$\mathrm{h}$ after irradiation from a total of six mice. Upon antigen unmasking, immunostaining with primary antibody (1:25 dilution) was performed using the rabbit Vectastain $\mathrm{ABC}$ Elite kit (Vector Laboratories) following the manufacturer's instructions. Vector 3,3'diaminobenzidine peroxidase substrate (Vector Laboratories) was used for color development. Stained sections were then dehydrated and counterstained with Vector Hematoxylin QS (Vector Laboratories), and immunoreactivity was assessed by at least two investigators.

\subsubsection{Monitoring the Radiation-Mitigating Effect of OTP on Survival}

Conscious eight-week-old female mice were injected with $0.5 \mathrm{mg} / \mathrm{kg}$ OTP or vehicle (2\% DMSO-PBS) 30 min before irradiation with 9 Gy (LD100/30) of $\gamma$ irradiation as described above. Each group consisted of 18 mice that provide $90 \%$ statistical power based on a Fisher's exact test with a one-sided 5\% significance level. The primary endpoint was survival to day 30 . The secondary endpoint was the mean survival time of decedents.

\subsubsection{Statistical Analysis}

Data are expressed as means $\pm \mathrm{SD}$ or SEM. Each in vitro experiment was repeated at least three times. For animal studies, each experimental group consisted of at least six mice; a minimum of eight KO mice was used per group. Student's t-test was used for comparing control and treatment groups. A p value of $<0.05$ was considered significant.

\subsection{RESULTS}

\subsubsection{Molecular Modeling and Pharmacological Characterization of OTP}

Complexes of OTP with the LPA receptor models are shown in Fig. 2.1B. The $\mathrm{LPA}_{1}$ complex shows a strong ionic interaction between the thiophosphate group and R3.28 (2.0 Å P-O:H-N distance). No interaction occurs involving Q3.29, a residue required for LPA binding and LPA-induced $\mathrm{LPA}_{1}$ receptor activation (Wang, 2001). Similar to the $\mathrm{LPA}_{1}$ complex, the $\mathrm{LPA}_{3}$ complex fails to show a strong hydrogen bonding interaction with Q3.29 due to the unfavorably nonlinear angle (Steiner, 2002) formed by the amide $\mathrm{N}-\mathrm{H}$ and the hydrogen bond acceptor $\left(112^{\circ}\right)$. However, a greater number of strong ionic interactions are observed in the $\mathrm{LPA}_{3}$ complex involving not only R3.28 but also K7.35 (2.2 ̊́ P-O:H-N and $2.4 \AA$ P-S:H-N distances, respectively). In contrast to our previous studies demonstrating a role for R5.38 in LPA-induced $\mathrm{LPA}_{3}$ activation (Fujiwara, 2005), OTP failed to interact with R5.38. The failure of OTP to interact with residues known to be required for LPA-induced activation of $\mathrm{LPA}_{1}$ and $\mathrm{LPA}_{3}$ is consistent with the observation of only partial agonism at these receptors (Fig. 2.1C). 
OTP shows both strong ionic interaction with R3.28 (1.6 § P-O: H-N distance) and moderate hydrogen bonding interaction with Q3.29 $\left(142^{\circ}\right)$. This hydrogen bonding angle is within the most highly populated cluster of angles observed in crystal structures ( Steiner, 2002). The $\mathrm{LPA}_{2}$ receptor lacks cationic amino acid residues at the top of TM7, so these two interactions comprise the required headgroup interactions for full agonism.

LPA and OTP activate LPA receptors stably expressed in RH7777 cells, providing a simple assay platform to study the pharmacological properties of these receptors as described in many previous reports (Fischer, 2001; Ohta, 2003; Virag, 2003; Durgam, 2005). OTP was compared in wild type, $\mathrm{LPA}_{1}, \mathrm{LPA}_{2}$, and $\mathrm{LPA}_{3}$-transfected RH7777 cells using $\mathrm{Ca}^{2+}$ mobilization as a measure of receptor activation (Fig. 2.1C). OTP was inactive in wild type RH7777 cells up to $30 \mu \mathrm{M}$, the highest concentration tested (data not shown). However, it activated all three EDG-family LPA receptors with varying potency and efficacy. OTP was always less potent than oleoyl LPA at all three receptors and was less efficacious at $\mathrm{LPA}_{1}$ and $\mathrm{LPA}_{3}$ receptors. At the $\mathrm{LPA}_{2}$ receptor subtype OTP was a full agonist and showed an apparent $\mathrm{EC}_{50}$ of $90 \mathrm{nM}$ compared $1 \mathrm{nM}$ for oleoyl LPA. Thus, OTP is a less efficacious full agonist of the $\mathrm{LPA}_{2}$ receptor subtype.

\subsubsection{Metabolic Resistance of OTP to Pancreatic Lipase and Lipid Phosphate Phosphatase 1}

The two major pathways of LPA breakdown include (phospho) lipase-mediated deacylation and lipid phosphate phosphatase-mediated dephosphorylation (Brindley, 2002). To determine the enzymatic stability of OTP by pancreatic lipase and LPP1 we synthesized $\left[{ }^{3} \mathrm{H}\right]$-OTP for monitoring the enzymatic hydrolysis followed by TLC separation of the products using previously established protocol (Kates, 1988; Yue, 2004). TLC analysis of the pancreatic lipase-mediated hydrolysis of $\left[{ }^{3} \mathrm{H}\right]-\mathrm{OTP}$ for up to $24 \mathrm{~h}$ showed neither the breakdown nor formation of the expected $\left[{ }^{3} \mathrm{H}\right]$-oleyl alcohol (data not shown). In contrast, LPA was fully metabolized under these conditions. The lack of cleavage is consistent with the requirement for the glycerol backbone that is absent in the OTP structure. LPP1 cleavage of $\left[{ }^{3} \mathrm{H}\right]$-OTP for up to $8 \mathrm{~h}$ showed no detectable dephosphorylation, indication that the thiophosphate moiety is considerably more resistant to cleavage by this enzyme than the phosphate moiety present in LPA.

\subsubsection{Absorption of Orally Applied OTP into the Blood}

The absorption of orally administered OTP into the bloodstream was determined by injecting $10^{6} \mathrm{dpm}\left[{ }^{3} \mathrm{H}\right]$-OTP tracer mixed with cold OTP to yield $1.5 \mathrm{mg} / \mathrm{kg}$ into the stomach. Blood samples collected at 60,90 , and 180 min later revealed that as little as $0.67 \% \pm 0.15,0.61 \% \pm 0.2$, and $0.45 \% \pm 0.15$ of the applied radioactivity appeared in the blood compartment. Assuming that all of it was non-metabolized OTP, this would yield a maximal blood concentration of $180 \mathrm{nM}$, which could activate LPA receptors. However, TLC analysis of the radioactively labeled compounds extracted from blood revealed no more than $0.01 \%$ of the injected material at the position of the authentic $\left[{ }^{3} \mathrm{H}\right]$-OTP 
standard, indicating that the little amount of radioactivity represents metabolites of the compound. The very low amount of $\left[{ }^{3} \mathrm{H}\right]$-OTP found in the blood after oral administration is reinforce by the lack of metabolism by pancreatic lipase and LPP1 present in the intestinal lumen, since these enzymes would produce the neutral oleyl alcohol, which could more effectively be taken up than a charged compound like OTP.

\subsubsection{Orally Applied OTP Is Non-toxic in the Effective Dose Range}

We tested OTP applied through oral gavage into the stomach of mice at 3,10 and $30 \mathrm{mg} / \mathrm{kg}$ complexed in $1 \mathrm{mM}$ BSA in PBS. Observation of the animals for $1 \mathrm{~h}$ after administration did not reveal any noticeable change in behavior and motor activity. Monitoring the weight-gain curves of the animals showed only a 24-h transient weight drop amounting to a mean of $3 \mathrm{~g}$ and only in the $30 \mathrm{mg} / \mathrm{kg}$ treatment group, but these animals rebounded by the second day and the median weight of the group was not significantly different from the vehicle treated controls beyond that up to 5 days the time the weight of the animals was followed daily. Thus, OTP showed no serious side effects even when administered at a 60-fold higher concentration - the highest dose tested so far - than the $0.5 \mathrm{mg} / \mathrm{kg}$, which was highly effective in protecting the life of animals from lethal doses of irradiation (see below).

\subsubsection{OTP Surpasses the Antiapoptotic Activity of LPA in IEC-6 Cells}

We previously showed that LPA protects intestinal epithelial cells from apoptosis induced by four different mechanisms (Deng, 2002, 2003 \& 2004). We compared the antiapoptotic effect of OTP with that of LPA. Although both OTP and LPA dosedependently protected IEC-6 cells from $\gamma$-irradiation-induced DNA damage, OTP at concentrations greater than $1 \mu \mathrm{M}$ conferred significantly higher protection (Fig. 2.2A). Both OTP and LPA applied at $10 \mu \mathrm{M}$ significantly protected IEC-6 cells from either topoisomerase inhibitor camptothecin- or the proinflammatory cytokine TNF- $\alpha$-induced DNA damage as compared to control $(p<0.001)$, and at this concentration OTP showed stronger antiapoptotic activity than did LPA ( $<<0.05$, Fig. 2.2B). OTP and LPA significantly inhibited caspase 3 and caspase 9 activity induced with $\gamma$-irradiation; again, the OTP-induced inhibition was significantly higher than that of LPA $(p<0.05$, Fig. 2.2C). Furthermore, analysis of caspase 3 activation by Western blotting demonstrated that both OTP and LPA significantly inhibited the conversion of the $32-\mathrm{kDa}$ procaspase into its active form, and OTP showed significantly higher inhibition compared to LPA (Fig. 2.2D. $\mathrm{p}<0.05$ ). Lastly, a brief 15-min OTP treatment inhibited the TNF- $\alpha$-induced activation of caspase 8 (Fig. 2.2E) that provides further support for its effect in reducing apoptosis elicited via the extrinsic pathway. These results together indicate that OTP mimics the antiapoptotic action of LPA in IEC-6 cells that express LPA 1 and LPA receptors (Deng, 2002). 
Figure 2.2. OTP is more effective in reducing apoptosis than LPA in IEC-6 cells. IEC-6 cells were washed twice and starved in serum-free DMEM overnight. (Panel A) OTP (circles) or LPA (squares), ranging from 0 to $30 \mu \mathrm{M}$, was applied $15 \mathrm{~min}$ before $25 \mathrm{~Gy} \gamma$-irradiation. DNA fragmentation was evaluated $18 \mathrm{~h}$ postirradiation as described under Methods. (Panel B) IEC-6 cells were treated with $10 \mu \mathrm{M}$ OTP or LPA before exposure to $20 \mu \mathrm{M}$ camptothecin or $20 \mathrm{ng} / \mathrm{ml} \mathrm{TNF}-\alpha$ plus $10 \mu \mathrm{g} / \mathrm{ml}$ cycloheximide. DNA fragmentation was evaluated $6 \mathrm{~h}$ later. Con designates non-treated control cells, Veh

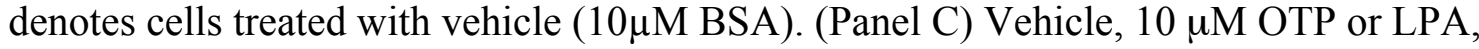
was applied to IEC-6 cells 15 min before 25 Gy -irradiation. Caspase 3 and caspase 9 activities were measured $18 \mathrm{~h}$ postirradiation by ELISA. (Panel D) Western blot analysis of caspase 3 activation in LPA- and OTP-treated (10 $\mu \mathrm{M}$ each) IEC-6 cells 15 min prior to $25 \mathrm{~Gy} \gamma$-irradiation. A total of $20 \mu \mathrm{g}$ cytosolic protein was loaded for each lane. (Panel E) A 15 -min OTP or LPA pretreatment inhibits $20 \mathrm{ng} / \mathrm{ml} \mathrm{TNF-} \alpha$ plus $10 \mu \mathrm{g} / \mathrm{ml}$ cycloheximide-induced caspase 8 activity. ${ }^{*} \mathrm{p}<0.01$ compared to the appropriate control group. Data shown are means \pm SD of at least three experiments. 

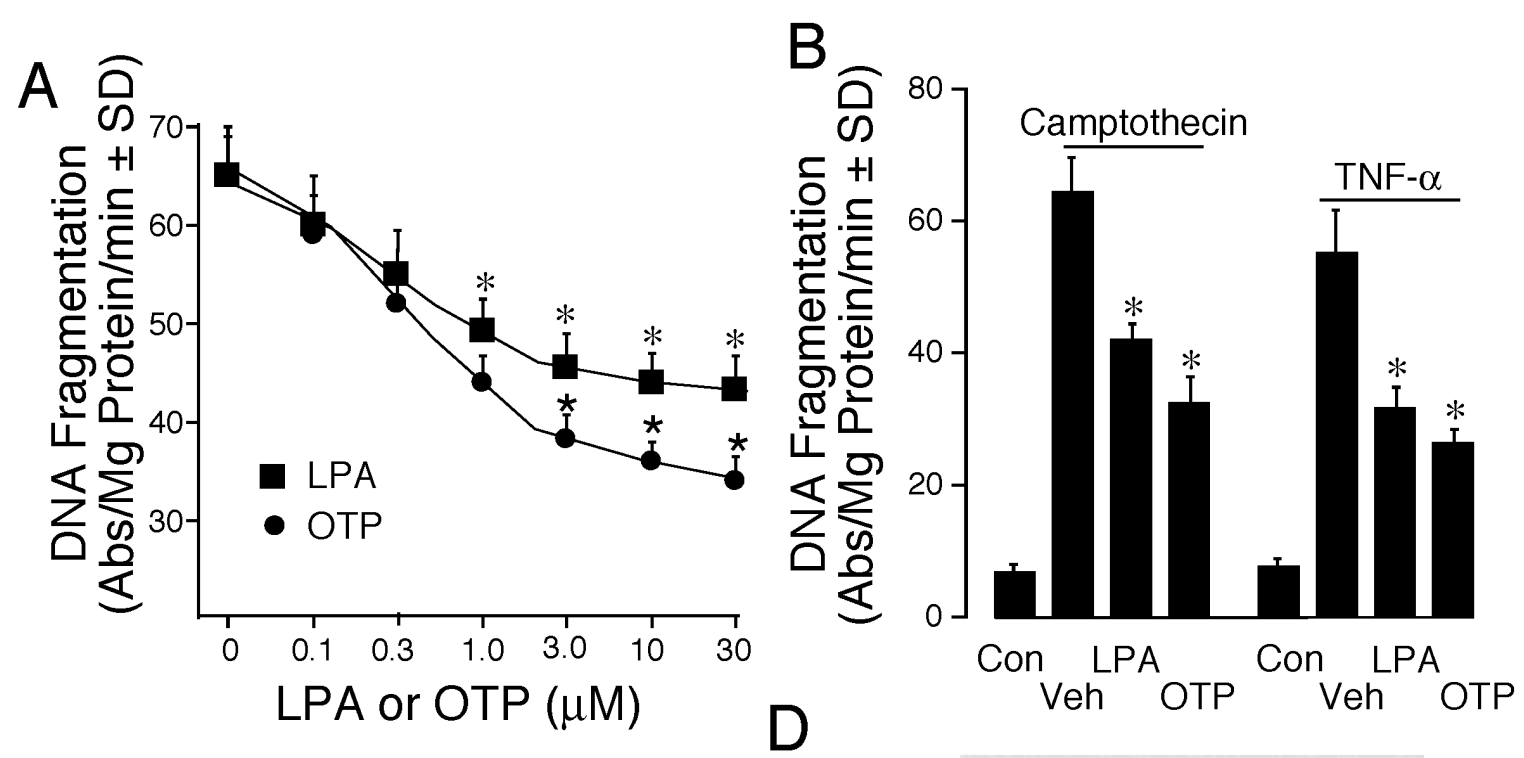

$\mathrm{C}$

Pro$\mathrm{kDa}$

Caspase $3---32$

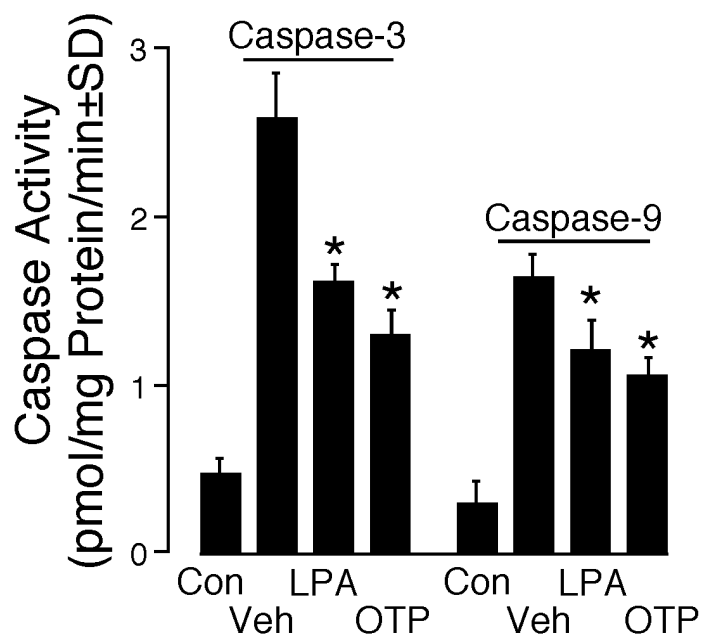

Active-

Caspase 3
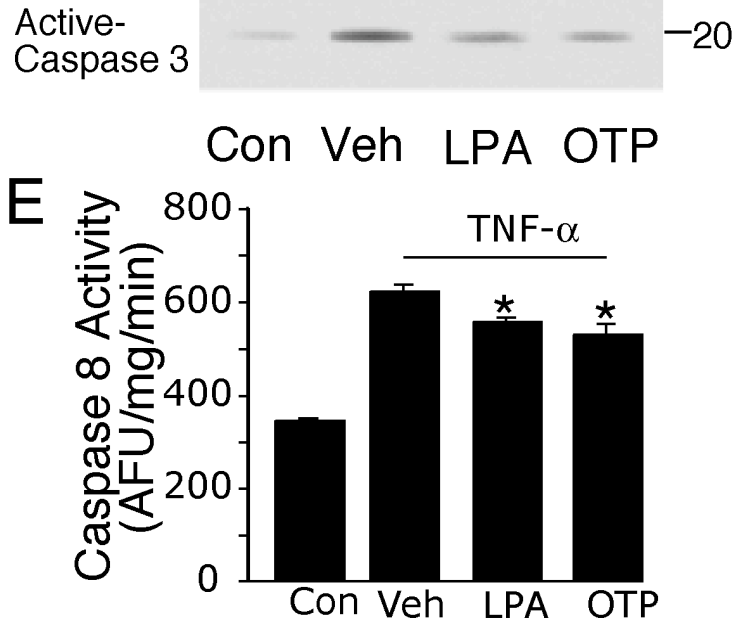


\subsubsection{The Antiapoptotic Activity of OTP Requires PTX-sensitive G Protein, Mitogen Activated Protein Kinase and PI3-Kinase/AKT Signaling}

We have previously shown that in IEC-6 cells the antiapoptotic action of LPA requires PTX-sensitive G protein, MEK, and PI3-kinase signaling (Deng, 2003). We found that OTP, just like LPA, activated ERK1/2 and p38 MAPK and AKT phosphorylation (Fig. 2.3A-C). We did not detect JNK phosphorylation under identical conditions (data not shown). To evaluate whether these same signaling mechanisms are required for OTP- elicited antiapoptotic activity, we examined these pathways by using pharmacological inhibitors and exposing these cells to OTP or LPA with or without 25 Gy $\gamma$-irradiation. In nonirradiated cells, just like LPA, OTP treatment for 5 min induced a significant increase in ERK1/2, p38, and AKT phosphorylation (Fig. 2.3). Blocking of ERK1/2 and AKT activation upstream by the MEK inhibitor PD98059 and PI3K inhibitor LY294002 completely abolished both OTP- and LPA-elicited activation, as well as protection (Fig. 2.3A, C, and D), indicating that the antiapoptotic activity of OTP required the activation of the ERK $1 / 2$ and AKT pathways. Pretreatment of IEC- 6 cells with PTX completely abolished LPA-elicited ERK1/2 and AKT activation (Fig. 2.3A and C). In agreement with the requirement for a PTX-sensitive G protein in the antiapoptotic mechanism, PTX cancelled LPA-elicited protection from $\gamma$-irradiation-induced DNA damage. In contrast, although PTX abolished OTP-induced AKT phosphorylation, it only partially attenuated ERK1/2 activation, indicating that OTP-stimulated ERK1/2 activation was mediated through not only PTX-sensitive G proteins. In support of this result, PTX pretreatment only partially reduced OTP-initiated protection, which remained statistically significant following treatment with this toxin ( $p<0.05$, Fig. 2.3D). These results indicate some similarities, as well as differences, between the signaling pathways used by OTP and LPA. Because $\mathrm{LPA}_{1}$ and $\mathrm{LPA}_{2}$ are the two main EDG family receptors expressed in IEC-6 cells (Deng, 2003) and also in the mouse intestine (Deng, 2002; Li, 2005a), we next compared the role of the individual LPA receptors in the LPA- and OTPelicited antiapoptotic response.

\subsubsection{OTP Selectively Protects LPA $A_{2}$ Transfectants from $\mathrm{TNF}$ - $\alpha$-induced Apoptosis}

RH7777 cells do not endogenously express the EDG-family LPA receptor but express low amounts of $\mathrm{LPA}_{5}$ transcripts (Lee, 2006). When LPA or OTP was applied to wild type RH7777 cells exposed to TNF- $\alpha$ plus cycloheximide to induce apoptosis, neither compound applied at $10 \mu \mathrm{M}$ attenuated DNA fragmentation (Fig. 2.4A). In contrast, in RH7777 cells individually transfected with $\mathrm{LPA}_{1}, \mathrm{LPA}_{2}$, or LPA 3 , DNA fragemtnation was significantly reduced by LPA pretreatment (Fig. 2.4A). OTP applied at $10 \mu \mathrm{M}$ showed no significant antiapoptotic activity in $\mathrm{LPA}_{1}$ or $\mathrm{LPA}_{3}$ stable transfectants but evoked highly significant protection in the $\mathrm{LPA}_{2}$ transfectants, surpassing the effect of that of $10 \mu \mathrm{M}$ LPA (Fig. 2.3A, $p<0.001$ ). It is important to note that OTP and LPA both activate $\mathrm{Ca}^{2+}$ transients in $\mathrm{LPA}_{1}, \mathrm{LPA}_{2}$, or $\mathrm{LPA}_{3}$ transfectants (Fig. 2.1C) but not in wild type RH7777 cells. Thus, the antiapoptotic effect of OTP is not simply linked to activation of receptor-elicited $\mathrm{Ca}^{2+}$ mobilization but to the activation 
Figure 2.3. Activation of prosurvival signaling pathways by LPA and OTP in IEC-6 cells. IEC-6 cells were pretreated with pertussis toxin (PTX, $50 \mathrm{ng} / \mathrm{ml}$ ) overnight, PD98059 $(20 \mu \mathrm{M})$ for $1 \mathrm{~h}$, or LY294002 $(10 \mu \mathrm{M})$ for $30 \mathrm{~min}$, followed by the addition of $10 \mu \mathrm{M}$ OTP or LPA. Activation of ERK1/2 (Panel A), P38 MAPK (Panel B), and $\mathrm{PKB} / \mathrm{AKT}$ (Panel C) was evaluated by Western blot 5 min after treatment with $10 \mu \mathrm{M}$ OTP or LPA. A total of $20 \mu \mathrm{g}$ lysate protein was loaded for each lane. (Panel D) IEC-6 cells were exposed to 25 Gy $\gamma$-irradiation 15 min after OTP or LPA treatment $(10 \mu \mathrm{M})$. DNA fragmentation was evaluated $18 \mathrm{~h}$ postirradiation. ${ }^{*} \mathrm{p}<0.05$ compared to irradiation alone. ${ }^{*} \mathrm{p}<0.01$ compared to irradiation alone. Data shown are means \pm SD of at least three experiments. 


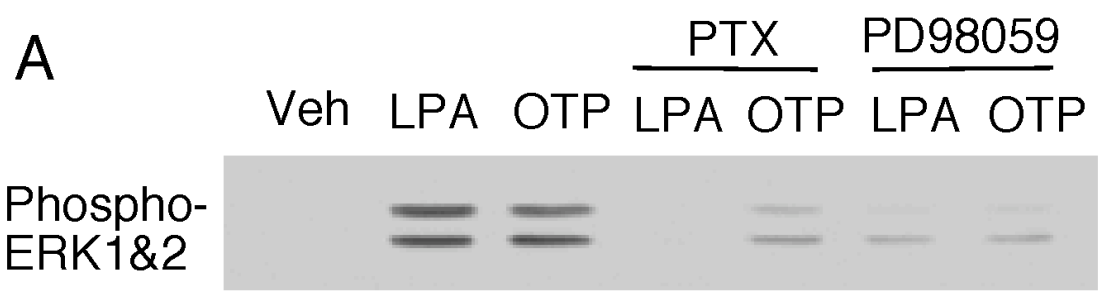

ERK1\&2

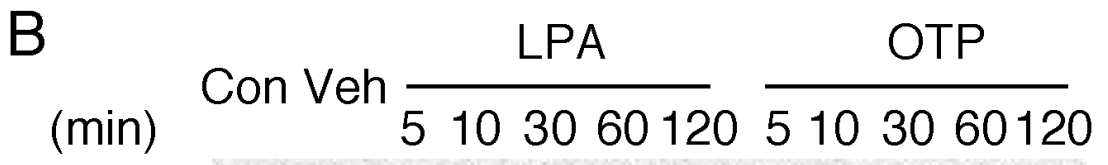
PhosphoP38

Actin

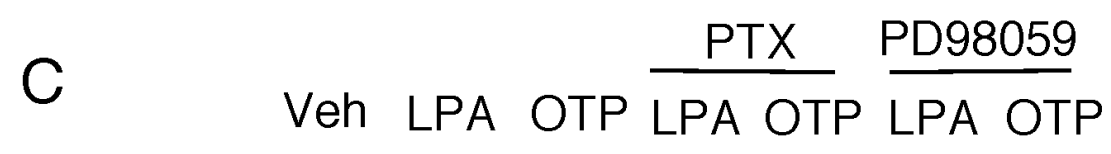

Phospho-

Akt

Akt

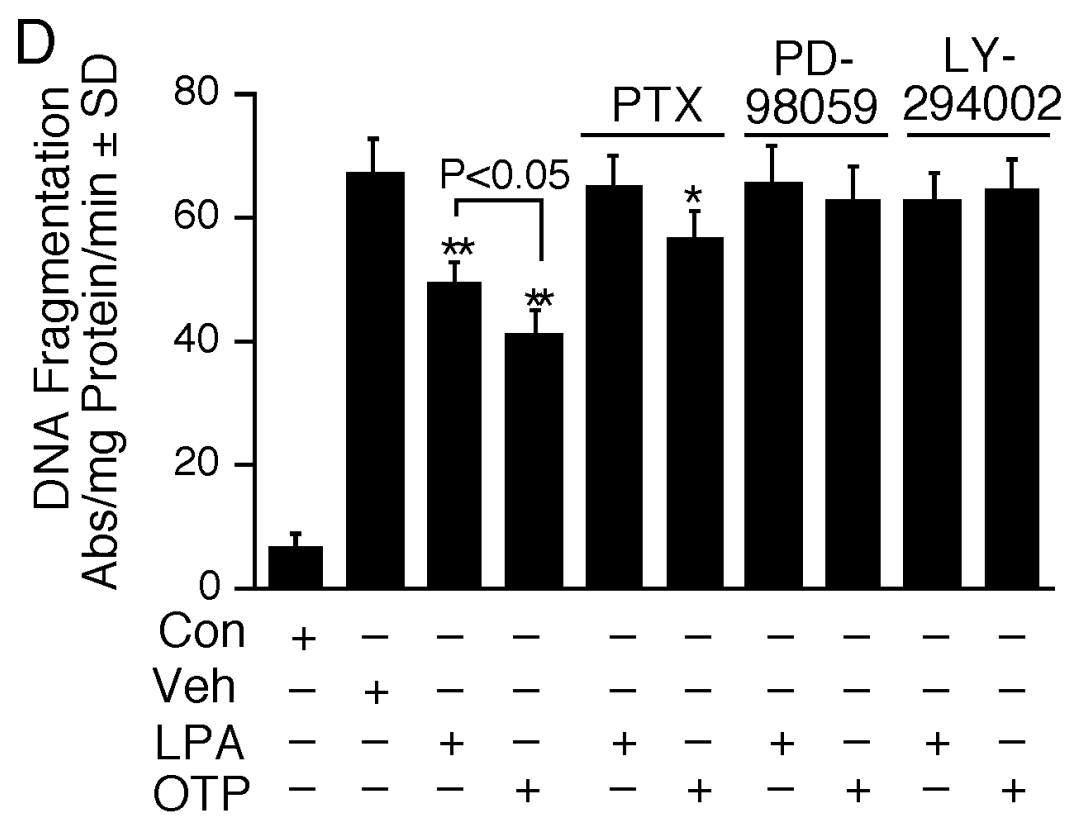


Figure 2.4. OTP selectively protects $L P A_{2}$ transfectants against TNF $\alpha / C H X-$ induced apoptosis. RH7777 cells were transfected with empty pCDNA3.1 vector or $\mathrm{LPA}_{1}, \mathrm{LPA}_{2}$, or LPA 3 and pre-incubated with $10 \mu \mathrm{M}$ OTP or LPA for $15 \mathrm{~min}$, followed by TNF- $\alpha(20 \mathrm{ng} / \mathrm{ml})$ plus CHX $(10 \mu \mathrm{g} / \mathrm{ml})$ exposure to induce apoptosis (Panel A). Note that whereas LPA reduced TNF $\alpha / \mathrm{CHX}$-induced DNA fragmentation in all three transfectants, OTP was effective only in $\mathrm{LPA}_{2}$ cells. Both ligands elicited $\mathrm{Ca}^{2+}$ transients in these same cell lines (cf. Fig. 2.1C). LPA 1 and LPA 2 transfectants (Panel B) were pretreated with pertussis toxin overnight (PTX, $50 \mathrm{ng} / \mathrm{ml}$ ), the MEK inhibitor PD98059 $(20 \mu \mathrm{M})$ for $1 \mathrm{~h}$, or the PI3K inhibitor LY294002 $(10 \mu \mathrm{M})$ for $30 \mathrm{~min}$, followed by the addition of $10 \mu \mathrm{M}$ OTP or LPA for $15 \mathrm{~min}$ and challenged with TNF- $\alpha(20 \mathrm{ng} / \mathrm{ml})$ plus $\mathrm{CHX}(10 \mu \mathrm{g} / \mathrm{ml})$. DNA fragmentation was evaluated $6 \mathrm{~h}$ after TNF $\alpha / / \mathrm{CHX}$ addition. The reduction in DNA fragmentation was partially sensitive to PTX in $\mathrm{LPA}_{2}$ cells and was abolished in $\mathrm{LPA}_{1}$ cells. ${ }^{*} \mathrm{p}<0.05, * * \mathrm{p}<0.001$ compared with TNF $\alpha / / \mathrm{CHX}$ alone. Data shown are means \pm SD of three experiments. 
A

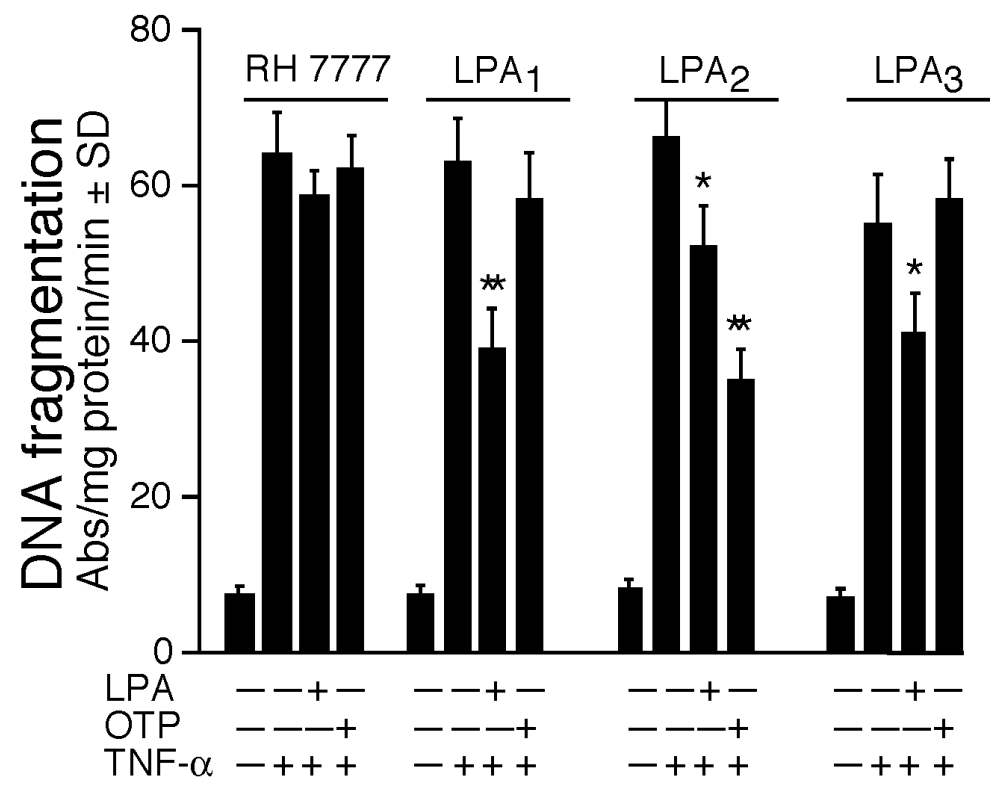

$\mathrm{B}$

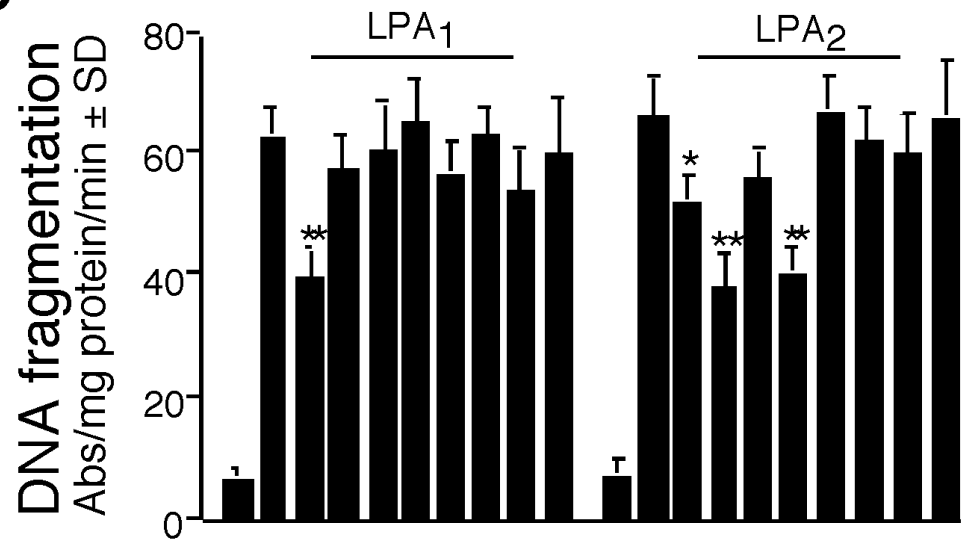

PTX $\quad---++-------++----$ LY-294002-- - - - + + - - - - - - + + PD98059 - - - - - + + - - - - - + + $\mathrm{LPA} \quad--+-+-+-+---+-+-+-+$ $\begin{array}{ll}\text { OTP } & ---+-+-+-+---+-+-+-+ \\ \text { TNF- } \alpha & -+++++++++-+++++++++\end{array}$ 
of more complex signals originating from a specific receptor subtype, which is $\mathrm{LPA}_{2}$. The protective effect of LPA in LPA 1 and LPA 2 transfected RH7777 cells was abolished by PTX and was cancelled by pharmacological blockade of MEK/ERK1/2 and PI3K/AKT activation, which mirrors our findings in IEC-6 cells (compare Fig. 2.4B and 2.3D). Just as in IEC-6 cells, the protective effect of OTP in RH7777 $\mathrm{LPA}_{2}$ transfectants was only partially PTX-sensitive and was completely abolished only by blocking ERK1/2 and PI3K/AKT pathways (Fig. 2.4B). These results identify $\mathrm{LPA}_{2}$ in this model system as the main target of OTP and pinpoint a mechanism that involves MEK/ERK1/2 and PI3K/AKT activation mediated through multiple PTX-insensitive and -sensitive $G$ proteins. $\mathrm{LPA}_{2}$ is known to be coupled to $\mathrm{G}_{\mathrm{q}}$ and it mediates ERK1/2 activation in part (Ishii, 2000), a finding consistent with the partial inhibitory effect of PTX on the apoptotic effect found in IEC6, as well as in LPA 2 transfected RH7777 cells. However, this finding does not explain why OTP, unlike LPA, failed to attenuate apoptosis in $\mathrm{LPA}_{1}$ - or $\mathrm{LPA}_{3}$-transfected RH7777 cells even though it activated these receptor subtypes indicated by the $\mathrm{Ca}^{2+}$-responses (Fig. 2.1C).

\subsubsection{OTP Attenuates Radiation-induced Apoptosis in the Intestine}

We have previously demonstrated that LPA given orally prevents apoptosis induced by $\gamma$-irradiation in the stem cell region of the intestinal crypt (Deng, 2002). Here, we sought to test whether OTP exerted a similar effect and compared its antiapoptotic efficacy with that of LPA by quantifying apoptotic bodies in the jejunal epithelium $4 \mathrm{~h}$ after 15 Gy $\gamma$-irradiation ( $\sim \mathrm{LD}_{100 / 10}$ ). In agreement with our previous report (Deng, 2002) using ICR animals was less than 0.5 per crypt-villus unit (Fig. 2.5A). In vehicle-treated $(100 \mu \mathrm{L}$ of $200 \mu \mathrm{M}$ BSA in PBS) wild type C57BL/6 mice, the number of apoptotic bodies per crypt-villus unit increased 6 -fold $4 \mathrm{~h}$ following $\gamma$-irradiation. Oral pretreatment with LPA ( $2 \mathrm{mg} / \mathrm{kg}$ into the stomach $2 \mathrm{~h}$ before irradiation) significantly reduced the number of apoptotic bodies per crypt-villus unit $(\mathrm{p}<0.01$, Fig. $2.5 \mathrm{~A})$. Oral OTP pretreatment (same timing and dose as LPA) reduced the number of apoptotic bodies by $50 \%$, which was significantly higher than that elicited by LPA $(p<0.05$, Fig. 2.5A). Analysis of the distribution of the apoptotic bodies along the crypt-villus unit showed that $\gamma$-irradiation resulted in a high frequency of apoptotic cells over the stem cell zone of the crypt, four to seven cell positions from the base (Fig. 2.5B). Both LPA and OTP significantly reduced the number of apoptotic cells over the stem cell zone in the irradiated animals.

Our data using the RH7777 heterologous over-expression model indicated that OTP elicited its antiapoptotic action through the $\mathrm{LPA}_{2}$ receptor subtype (Fig. 2.4). To evaluate which LPA receptor mediates the protective effect of OTP in vivo, we performed the same treatments in $\mathrm{LPA}_{1}$ and $\mathrm{LPA}_{2}$ knockout mice. We detected no differences in the basal count of apoptotic bodies between wild type C57BL/6 mice and $\mathrm{LPA}_{1}$ or $\mathrm{LPA}_{2} \mathrm{KO}$ mice on this same genetic background. Surprisingly, $\gamma$-irradiation induced a significantly higher rate of apoptosis at the base of the crypt that includes a small number of stem cells in $\mathrm{LPA}_{2}$ knockouts compared to that in wild type mice, whereas there was no difference between wild type and $\mathrm{LPA}_{1}$ mice (Fig. 2.5A). LPA and 
Figure 2.5. OTP inhibits apoptosis in small intestinal epithelium of mice following $\gamma$-irradiation. Wild type C57BL/6 (black bars) and LPA (grey bars) or LPA $\mathrm{LO}_{2} \mathrm{KO}$ mice (open bars) were given vehicle orally $(100 \mu 1200 \mu \mathrm{M}$ BSA control), LPA ( $2 \mathrm{mg} / \mathrm{kg}$ ) or OTP $(2 \mathrm{mg} / \mathrm{kg}) 2 \mathrm{~h}$ before subjecting them to $15 \mathrm{~Gy}$ whole body $\gamma$-irradiation. Animals were sacrificed $4 \mathrm{~h}$ after irradiation to evaluate apoptosis in the small intestine by H\&E staining. (Panel A) The mean number of apoptotic cells per crypt-villus unit. Both LPA and OTP caused a statistically significant decrease in the number of apoptotic bodies compared to the vehicle-treated animal group $(\mathrm{p}<0.01)$. Furthermore, OTP was significantly more effective than LPA $(* p<0.05)$. The number of apoptotic bodies was significantly higher in the $\mathrm{LPA}_{2} \mathrm{KO}$ mice compared to wild type or $\mathrm{LPA}_{1}$ mice $\left({ }^{*} \mathrm{p}<\right.$ 0.01). (Panel B) Correlation between apoptotic cell index (percentage of apoptotic cells at a given cell position) and their positions along the crypt-villus axis. Cells 1-2 are Paneth cells in the graph. $\mathrm{n}=6$ animals in each group, and a minimum of 100 crypt-villus units were scored in each group. (Panel C) Reverse-transcription PCR analysis of LPA GPCRs in jejunum isolated from wild-type, $\mathrm{LPA}_{1}{ }^{-/-}$, and $\mathrm{LPA}_{2}{ }^{-/-}$mice. 

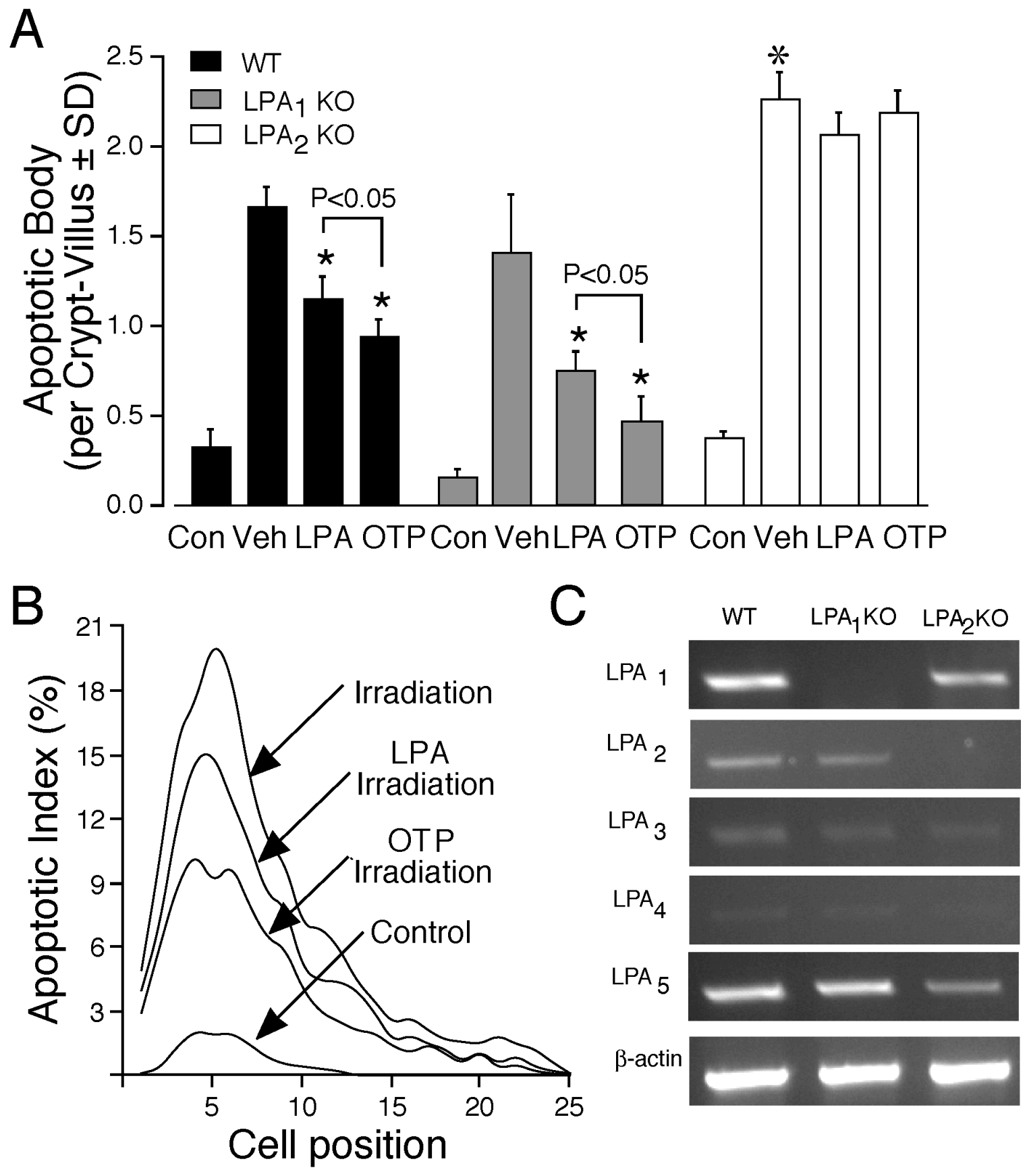
OTP had the same protective effect in wild type and LPA 1 mice. In contrast, LPA and OTP failed to attenuate $\gamma$-irradiation-induced apoptosis in $\mathrm{LPA}_{2} \mathrm{KO}$ mice. In wild type animals the biggest radiation-induced increase in apoptotic bodies was found in the stem cell region of the crypt (Fig. 2.5B). LPA and OTP caused the biggest decrease in the number of apoptotic cells in this region of the crypt suggesting that the cellular targets of LPA and OTP are the stem cells. RT-PCR analysis of the LPA receptors showed that all but $\mathrm{LPA}_{4}$ transcripts were expressed in the tissue (Fig. 2.5C). The LPA 1 and $\mathrm{LPA}_{2} \mathrm{KO}$ animals showed no compensatory change in the expression of the other LPA receptors. Thus the in vivo data are consistent with our findings that $\mathrm{LPA}_{2}$ is required for the antiapoptotic effect of OTP. Nonetheless, these data differ from the findings in the case of LPA and point to the essential role of $\mathrm{LPA}_{2}$ in mediating the in vivo antiapoptotic effect of LPA. Furthermore, the increased rate of apoptosis in $\mathrm{LPA}_{2} \mathrm{KO}$ mice suggests that this receptor may play a physiological role in the radiation sensitivity of the small intestine. These results also point to the fundamentally different role of the two LPA receptor subtypes in radiation sensitivity and apoptotic protection.

Next, we sought to obtain direct evidence that LPA and OTP treatments activate the same signal transduction mechanisms we have identified. First, we examined whether LPA and OTP attenuate the activation of the main executioner, caspase 3 , as we have seen in IEC-6 cells (Fig. 2.1C). Jejunum and ileum sections were immunostained with an antibody specific for the activated form of caspase 3, and the number of positive cells was counted in a minimum of 100 crypt-villus units in slides prepared from groups of four animals. In the nonirradiated vehicle-treated group, we could not detect caspase 3 positive cells, possibly due to the low amount of antigen present in cells naturally undergoing apoptosis combined with their low incidence in the healthy small intestine. In contrast, in the vehicle-treated irradiated group, caspase 3 positive cells were readily detected. Both LPA and OTP treatments significantly reduced the number of activated caspase 3 positive cells by $\sim 60 \%$ (Fig. $2.6 \mathrm{~A}, \mathrm{p}<0.05$ ). The inhibition of caspase 3 activation by LPA and OTP was also confirmed in intestinal epithelial lysates by measuring enzymatic activity (Fig. 2.6B). These experiments revealed that, in control nonirradiated animals, a low level of caspase 3 substrate cleaving activity is present, which increased 4-fold after 15 Gy $\gamma$-irradiation. LPA and OTP reduced caspase 3 activity significantly, although the inhibitory effect of OTP was greater.

Our previous and present studies using IEC-6 and other types of cells (Deng, 2003 $\& 2004$ ) have established the requirement of ERK1/2 and AKT activation in the antiapoptotic response to LPA and OTP. We next determined whether these pathways were activated in intestinal cell homogenates prepared from animals that have been treated with the two ligands by oral gavage. As shown in Fig. 2.6 C-D, both agents activated ERK1/2 and AKT phosphorylation as early as 30 min after administration in nonirradiated animals. However, the effect of OTP was considerably longer lasting in the tissue compared to the effect of LPA, which was absent in the samples collected $60 \mathrm{~min}$ after administration.

Studies using IEC-6 cells showed that LPA upregulated the expression of the antiapoptotic Bcl-2 mRNA and had no effect on Bcl-X $\mathrm{X}_{\mathrm{L}}, \mathrm{BAD}$, and Bak mRNA 
Figure 2.6. OTP and LPA reduces caspase 3 activation and activates prosurvival pathways in vivo. C57BL/6 mice were pretreated with $2 \mathrm{mg} / \mathrm{kg}$ LPA or OTP for $2 \mathrm{~h}$ and subjected to $15 \mathrm{~Gy}$ radiation exposures. Mice were sacrificed $4 \mathrm{~h}$ after radiation. (Panel A) Quantification of active caspase 3 immunoreactive cells. Paraffin-embedded jejunum sections from animals treated with radiation and pretreated with either LPA or OTP were stained with a rabbit polyclonal active caspase 3 antibody and fluorescein-labeled secondary antibody using indirect immunofluorescence as described under Methods, and the sections were counterstained with DAPI. Active caspase 3 immunoreactive cells were counted in a minimum of 100 crypt-villus units in groups of four animals in the groups. Both agents significantly reduced the number of activated caspase 3 positive cells ( $p<$ 0.05 ) when compared with vehicle-treated controls. (Panel B) Caspase 3 activity was determined in epithelial cell lysates prepared from the same animals whose jejunum segments were used for activated caspase 3 immunostaining in panel A. LPA and OTP both significantly reduced caspase 3 activity in the tissue lysates, a finding in agreement with the reduced number of active caspase 3 cells. (Panels C-D) Jejunum tissue from mice orally treated with vehicle, $2 \mathrm{mg} / \mathrm{kg} \mathrm{LPA}$, or OTP for the indicated times were homogenized and lysates analyzed using Western blotting with anti-phospho-ERK1/2 (C) or anti-phospho-AKT antibodies (D) and appropriate antibodies to the nonphosphorylated forms of the kinases to monitor equal loading. Note that the activation of both kinases was more robust and longer lasting to OTP compared to LPA. 


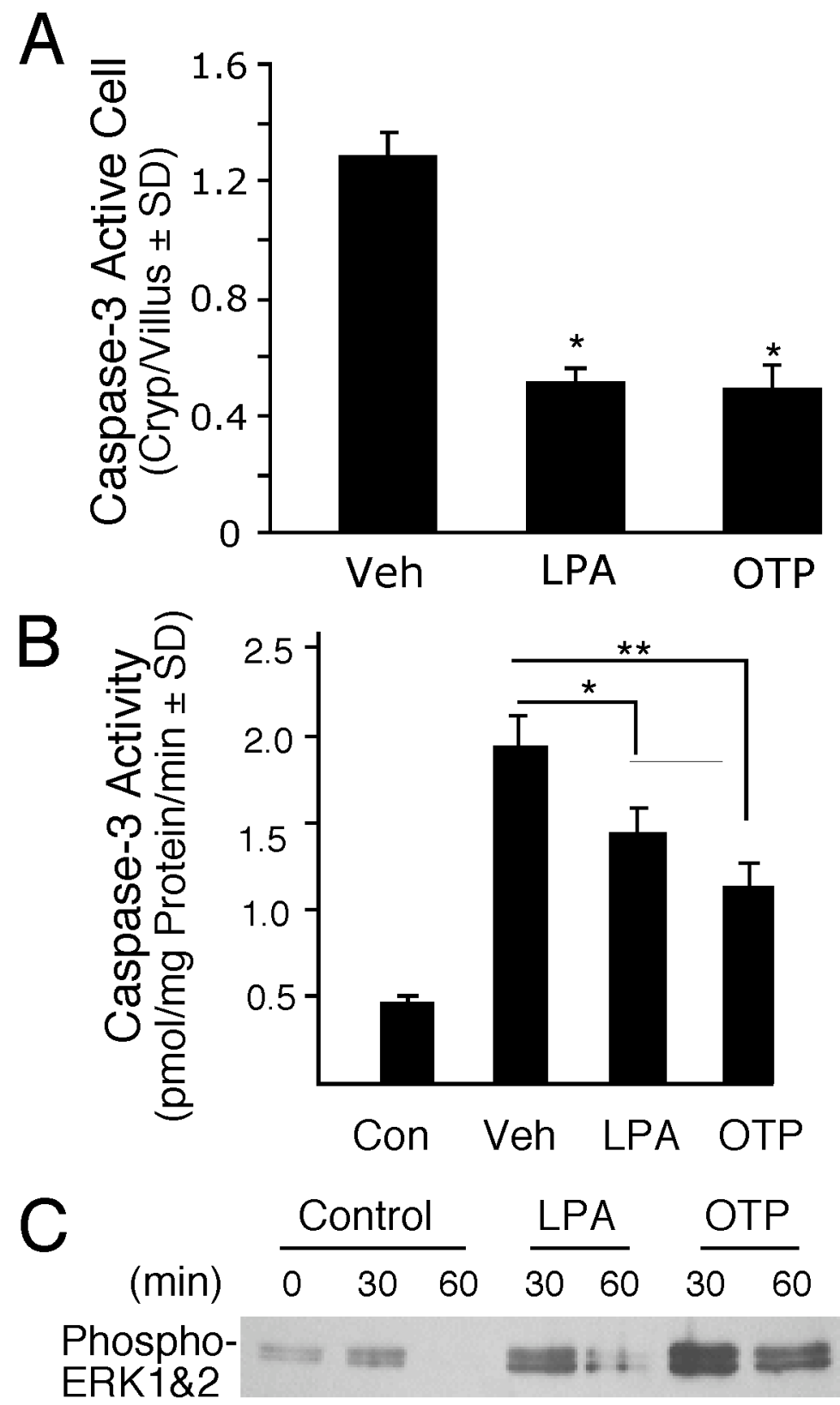

ERK1\&2

D

(min) $\frac{\text { Control }}{0 \quad 30 \quad 60} \quad \frac{\text { LPA }}{30 \quad 60} \quad \frac{\text { OTP }}{30 \quad 60}$

PhosphoAkt

Actin 
from LPA- and OTP-treated mice $4 \mathrm{~h}$ after irradiation (6h after treatment), we could not detect a significant change in Bcl- $\mathrm{X}_{\mathrm{L}}$ or Bcl-2 expression (data not shown). However, immunostaining with a Bcl- $\mathrm{X}_{\mathrm{L}}$ specific antibody revealed marked increases in LPA and OTP treated animals (Fig. 2.7) compared to jejunum and ileum (not shown) from the vehicle-treated animals. OTP treatment caused the most intense immunostaining (Fig. 2.7C), although LPA also elicited a substantial increase in the immunoreactivity (Fig. 2.7B). When we tested the level of in $\mathrm{Bcl}-\mathrm{X}_{\mathrm{L}}$ or Bcl-2 protein in OTP-treated camptothecin-challenged IEC-6 cells a transient increase in Bcl- $\mathrm{X}_{\mathrm{L}}$ but not in Bcl-2 was detected (Fig. 2.7D). These results taken together establish strong similarities, as well as some differences, between the prosurvival signals elicited by these two agents in cultured cells and the small intestinal tissue in vivo.

\subsubsection{OTP and LPA Enhance Intestinal Crypt Survival after Radiation Injury}

$\gamma$-Irradiation induces apoptosis in crypt cells, which in turn reduces the regenerative or clonogenic potential leading to the disruption of the barrier and absorptive function in the injured gut. We examined the effect of LPA and OTP on crypt survival in irradiated mice using $\mathrm{H} \& \mathrm{E}$ staining and BrdU incorporation to monitor regenerating $\mathrm{S}$ phase enterocytes. Irradiation with a dose of 15 Gy caused a $90 \%$ reduction in the number of crypts in jejunum in wild type mice within 4 days (Figs. 2.8 and 2.9). Oral LPA or OTP pretreatment enhanced intestinal crypt survival in a dose-dependent manner (Fig. 2.8A). OTP treatment of mice with doses above $0.2 \mathrm{mg} / \mathrm{kg}$ significantly enhanced crypt survival, while LPA treatment required a minimum dose of $1.0 \mathrm{mg} / \mathrm{kg}$ to cause a significant protection in crypt survival ( $<<0.05$, Fig. 2.8A). OTP at doses above $1 \mathrm{mg} / \mathrm{kg}$ maintained a significantly greater number of crypts than did LPA ( $p<0.05$, Fig. 2.8A). Followed by $15 \mathrm{~Gy} \gamma$-irradiation, OTP orally applied at $2 \mathrm{mg} / \mathrm{kg}$ increased the number of surviving crypts in the jejunum from 10 crypts to an average of 27 crypts per circumference (Fig. 2.8A). To further validate $\mathrm{LPA}_{2}$ as the target of the antiapototic action of LPA and OTP, we also examined crypt survival in $\mathrm{LPA}_{2} \mathrm{KO}$ mice treated the same way as their wild type counterparts described above. In contrast to irradiated wild type mice, $\mathrm{LPA}_{1}$ and particularly $\mathrm{LPA}_{2} \mathrm{KO}$ mice showed lower crypt survival. However, in LPA $_{1}$ KO mice LPA and OTP both elicited significantly increased crypt survival, which was not significantly different from that seen in wild type mice treated the same manner. $\mathrm{LPA}_{2} \mathrm{KO}$ mice revealed significantly higher radiation sensitivity. Whereas in wild type mice exposed to a 15 Gy dose the mean crypt survival per circumference was 10, in $\mathrm{LPA}_{2} \mathrm{KO}$ mice it was as little as 1 crypt/circumference (Fig. 2.8B). Neither LPA nor OTP applied at $2 \mathrm{mg} / \mathrm{kg}$, the highest dose tested, showed any effect in enhancing intestinal crypt survival in the $\mathrm{LPA}_{2} \mathrm{KO}$ mice. This observation extends the increased rate of apoptosis, the lack of treatment-induced reduction in apoptotic cells observed in the $\mathrm{LPA}_{2} \mathrm{KO}$ mice, ending strong support to the hypothesis that $\mathrm{LPA}_{2}$ is the molecular target of radioprotective effect of LPA and OTP. 

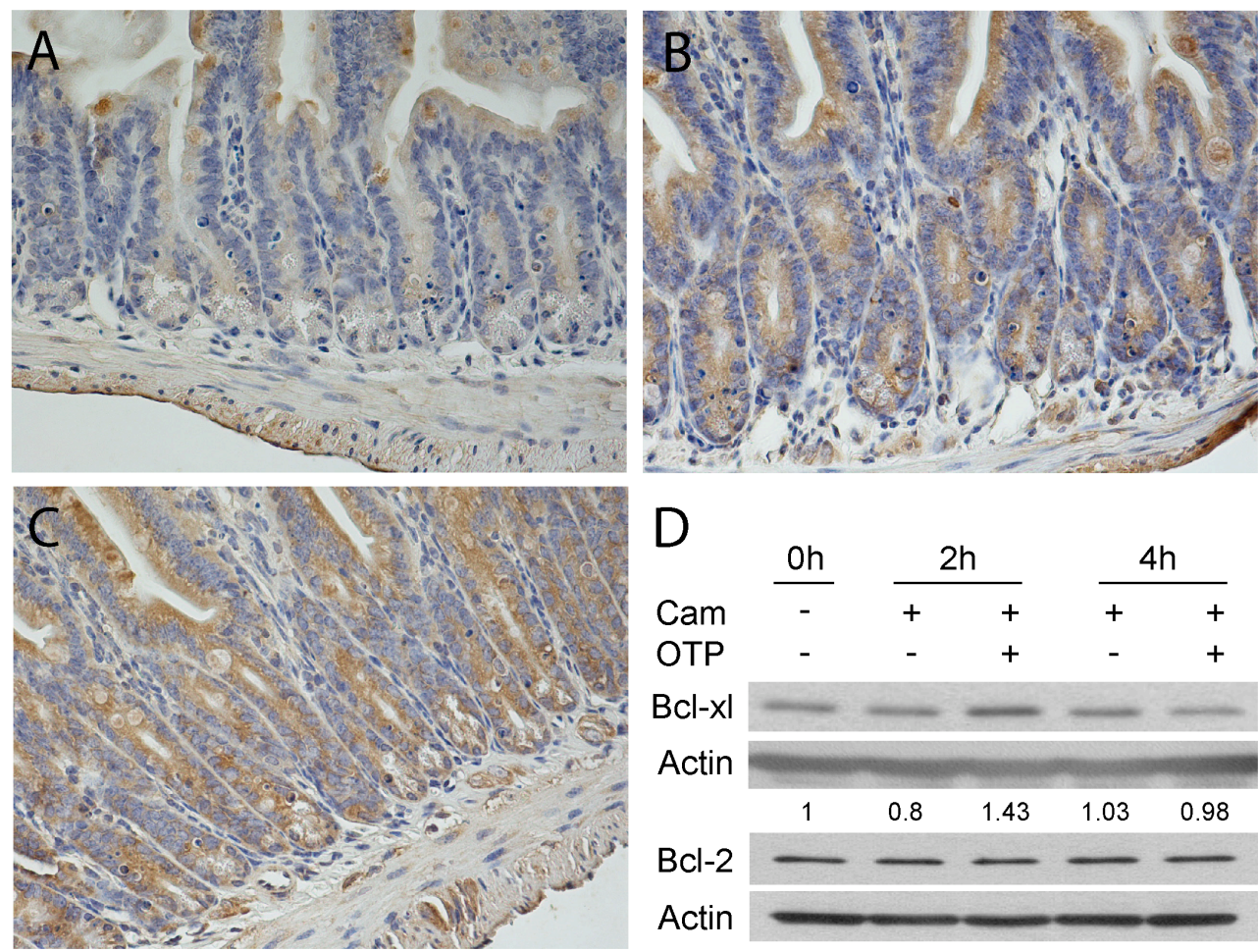

Figure 2.7. Immunohistochemical localization of antiapoptotic Bcl- $X_{L}$ protein in vehicle (200 $\mu \mathrm{M}$ BSA), LPA- and OTP-pretreated, irradiated small intestinal sections of C57BL/6 mice. Sections of vehicle-treated mice (A) showed less Bcl- $\mathrm{X}_{\mathrm{L}}$ expression compared to sections obtained from mice treated with $2 \mathrm{mg} / \mathrm{kg}$ LPA (B) or OTP (C). Calibration bar is $200 \mu \mathrm{m}$. The patterns shown represent sections obtained from all mice in the corresponding group of four mice per treatment. Panel C. OTP increases the cytoplasmic level of Bcl- $\mathrm{X}_{\mathrm{L}}$ but not Bcl-2 in camptothecin-treated IEC-6 cells. After overnight serum starvation, IEC-6 cells were pretreated with $10 \mu \mathrm{M}$ OTP or vehicle for $1 \mathrm{~h}$ prior to challenge with $20 \mu \mathrm{M}$ camptothecin (Cam). A $20 \mu \mathrm{g}$ cytoplasmic protein was loaded for each lane and blotted with anti- Bcl- $\mathrm{X}_{\mathrm{L}}$ or Bcl-2 antibodies as described under Materials and Methods. Note that OTP increased the amount of Bcl- $\mathrm{X}_{\mathrm{L}}$ but not of Bcl-2 that peaked at $2 \mathrm{~h}$ after camptothecin and decreased to control level by $4 \mathrm{~h}$. 
Figure 2.8. Clonogenic assays reveals increased intestinal crypt survival in OTPand LPA-treated mice following irradiation. (Panel A) Wild type C57BL/6 mice were given vehicle (BSA $200 \mu \mathrm{M})$, LPA, or OTP (0 to $2 \mathrm{mg} / \mathrm{kg}$ ) orally $2 \mathrm{~h}$ before being subjected to 15 Gy whole body $\gamma$-irradiation. (Panel B) Wild type C57BL/6 (black bars), $\mathrm{LPA}_{1} \mathrm{KO}$ (grey bars) or LPA $2 \mathrm{KO}$ mice (open bars) were orally given vehicle, LPA, or OTP, $2 \mathrm{mg} / \mathrm{kg}, 2 \mathrm{~h}$ before 15 Gy $\gamma$-irradiation, and the animals were sacrificed 4 days after irradiation. Crypt survival was evaluated by H\&E staining combined with BrdU immunostaining. $n=6$ in each group. Data are expressed as means \pm SD of surviving crypts per cross section. The level of significance based on Student $t$ test was $* \mathrm{p}<0.05$, or $* * p<0.01$, between the designated groups, and was $\# p<0.01$ compared to irradiation alone and between the mean crypt survival in wild type and $\mathrm{LPA}_{1}$ and $\mathrm{LPA}_{2} \mathrm{KO}$ mice. 
A

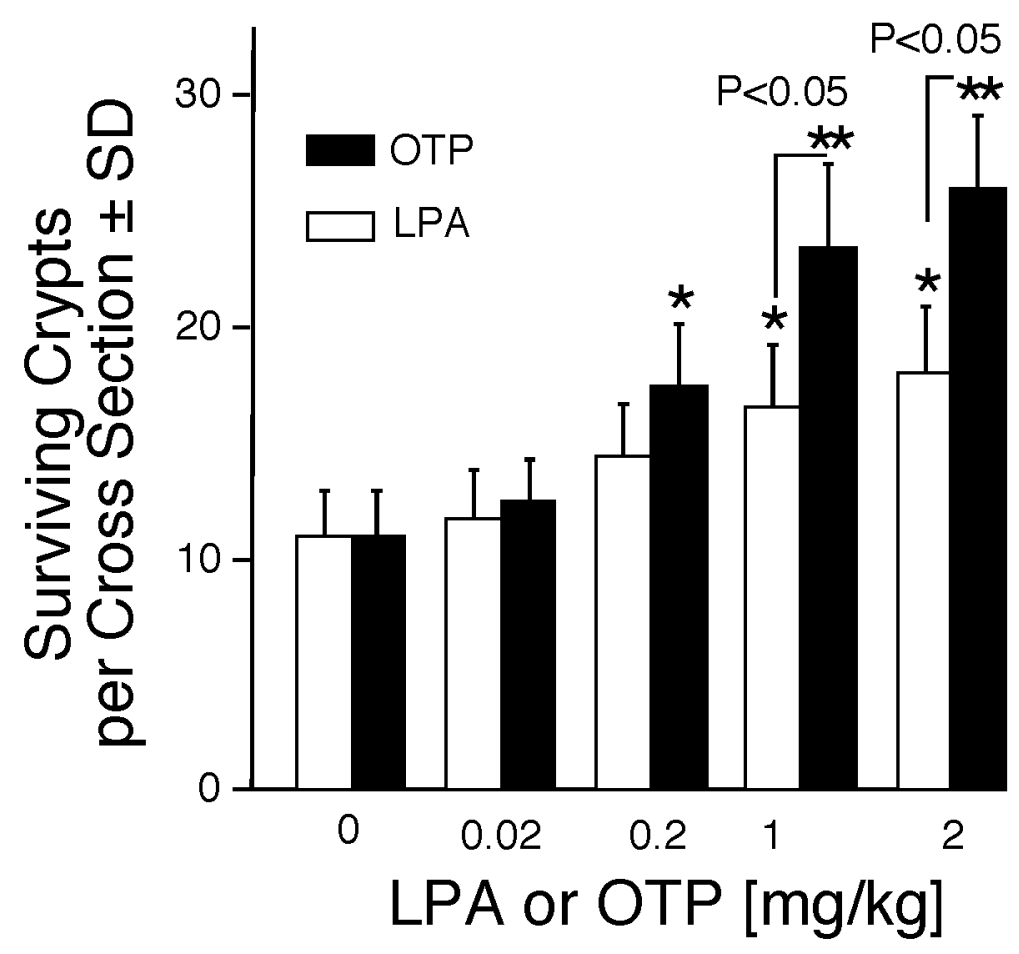

B

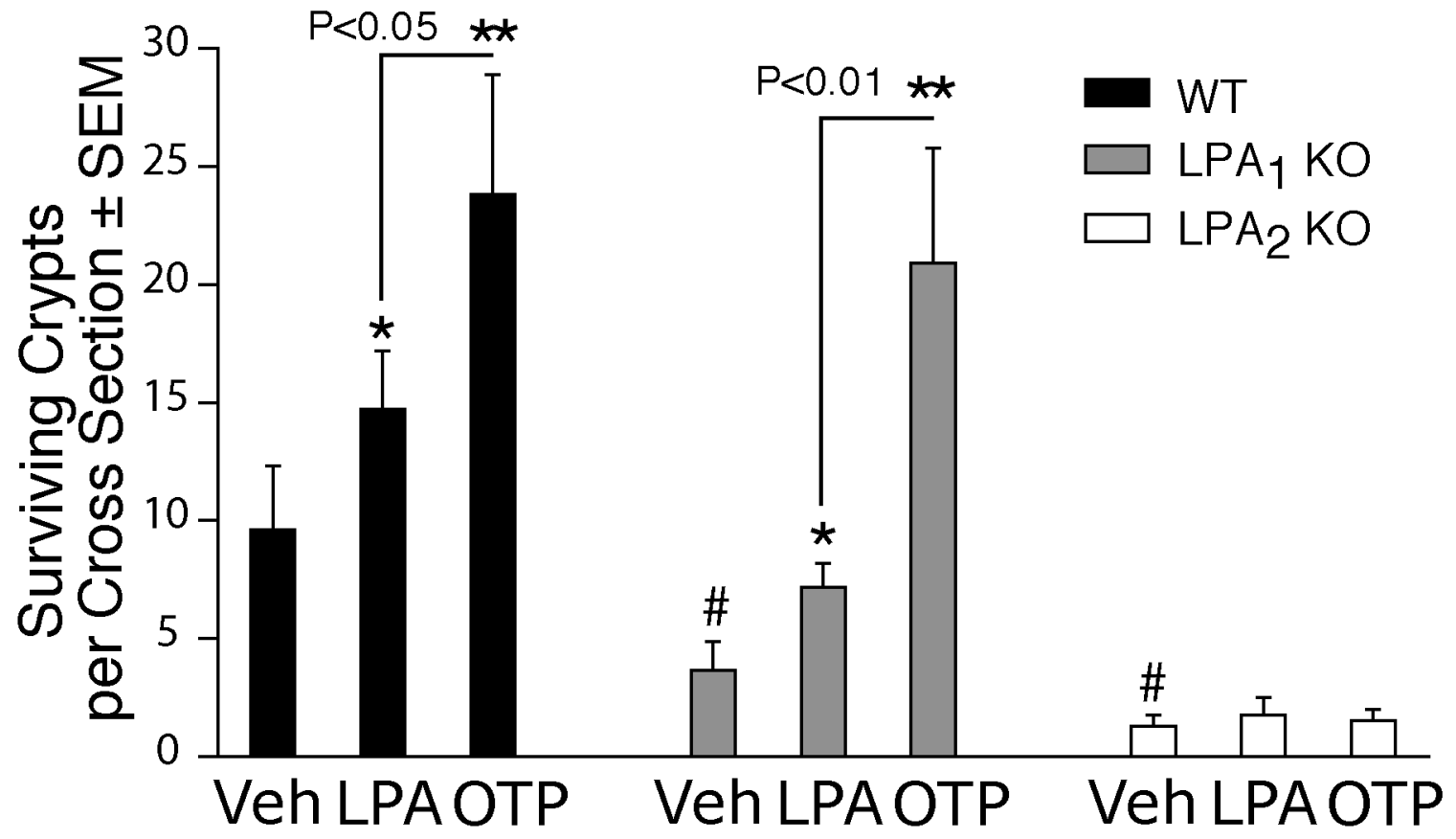



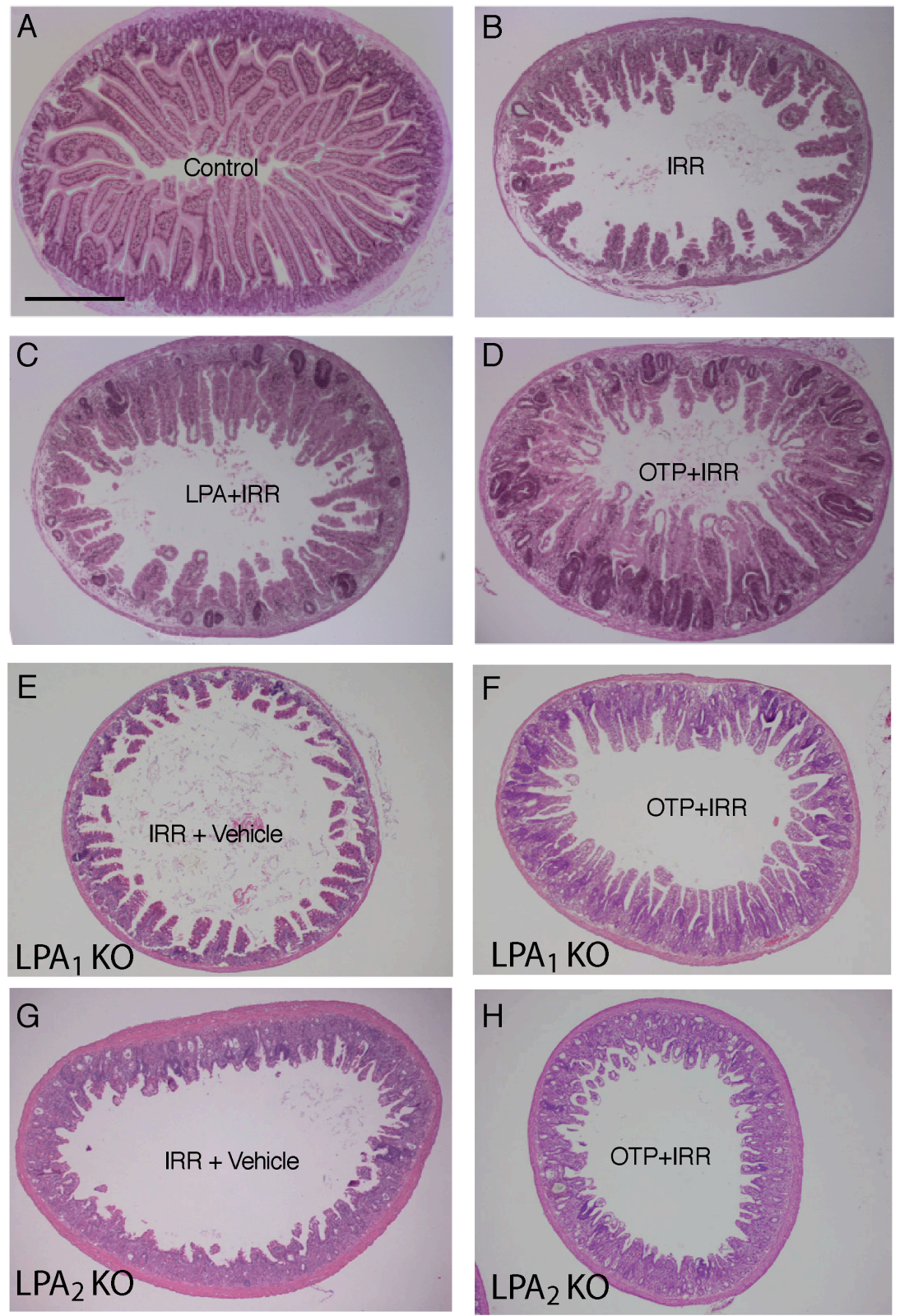

Figure 2.9. Representative H\&E stained gut cross-sections from treated $L P A_{1}$ and LPA $_{2}$ KO mice sacrificed 4 days after irradiation. Nonirradiated control (A), irradiated vehicle-treated (B), LPA-treated (C, $2 \mathrm{mg} / \mathrm{kg})$, and OTP-treated WT (D, 2 $\mathrm{mg} / \mathrm{kg}$ ), vehicle treated $\mathrm{LPA}_{1}$ and $\mathrm{LPA}_{2} \mathrm{KO}$ (panels E \& G) and OTP $(2 \mathrm{mg} / \mathrm{kg})$ treated $\mathrm{LPA}_{1} \mathrm{KO}(\mathrm{F})$ and $\mathrm{LPA}_{2} \mathrm{KO}(\mathrm{H})$ mice. Calibration bar is $\sim 500 \mu \mathrm{M}$. 


\subsubsection{OTP Reduces Radiation-induced Death}

Although orally applied OTP did not get absorbed into the systemic circulation in an effective concentration but reduced radiation-induced cell death in the small intestine we reasoned that if administered systemically, it might exert a protective effect on survival. To test this hypothesis we turned to an intraperitoneal route of administration and monitored $30 \mathrm{~d}$ survival as the primary endpoint following a 9 Gy dose of $\gamma$ irradiation, which in our system approximately equals a $\mathrm{LD}_{100 / 30}$ (Fig. 2.10). The group of mice that received a $0.5 \mathrm{mg} / \mathrm{kg}$ dose $30 \mathrm{~min}$ prior to irradiation, we observed a 50 per cent survival to the end of the experiment whereas, all animals in the vehicle treated group died. These results expand the potential usefulness of OTP from the topical protection of the small intestine following oral route of administration to a radiomitigating agent effectively reducing death caused by the hematopoietic syndrome.

\subsection{DISCUSSION}

The objective of the present study was to evaluate the effect of OTP, a rationally designed metabolically stabilized LPA mimic, on radiation-induced apoptosis in vitro and in vivo. Our data indicate that OTP is a highly effective antiapoptotic agent that engages prosurvival pathways similar to those elicited by LPA through the LPA 2 receptor subtype. OTP shares a pharmacological profile similar to that of LPA in that it activated the three EDG family LPA receptors expressed heterologously in RH7777 cells. The rank order of OTP's EC 50 values was $\mathrm{LPA}_{2}(90 \mathrm{nM})<\mathrm{LPA}_{3}<\mathrm{LPA}_{1}$ based on $\mathrm{Ca}^{2+}$ transients

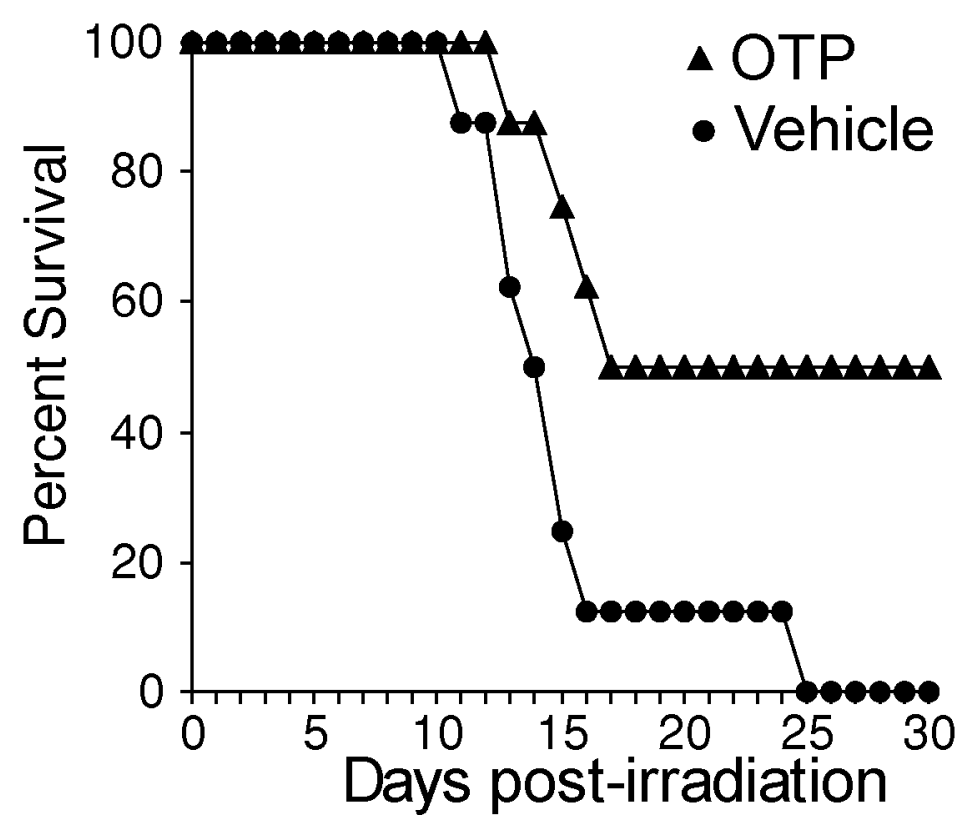

Figure 2.10. OTP administered intraperitoneally 30 minutes prior to irradiation with a 9 Gy dose $\left(\mathrm{LD}_{100 / 30}\right)$ increased 30-day survival. 
elicited in this heterologous expression system. These values render OTP a considerably weaker ligand of the three LPA receptors compared to LPA 18:1. OTP when compared to LPA in three different apoptosis models, which included radiation- and camptothecinelicited DNA damage-induced and TNF- $\alpha / \mathrm{CHX}$-elicited extrinsic mechanisms, always surpassed the protective effect of LPA. In agreement with the cellular models of apoptosis, OTP was more effective in reducing the number of apoptotic bodies, caspase 3 positive cells, and caspase 3 activity in C57BL/6 mice exposed to an $\mathrm{LD}_{100 / 15}$ dose of $\gamma$ irradiation.

What may account for the higher efficacy of OTP relative to LPA in spite of its weaker pharmacological potency? Although we cannot pinpoint a single reason for the increased efficacy of OTP, there are major differences in its metabolic resistance and bioavailability compared to LPA. First, we found that OTP was not cleaved by pancreatic lipase, the major lipase in the intestine. Second, it is not degraded by lipid phosphate phosphatase 1, which is the other major mechanism for the inactivation of LPA (Brindley, 2002). Third, due to the lack of a glycerol backbone, unlike LPA, OTP cannot be acylated by lysophosphatidate transacetylases. Fourth, likely due to its polar character, OTP does not transverse the cell membrane readily, and we could not detect radioactively labeled OTP in the blood of experimental animals following application via oral gavage. These differences in its metabolism and bioavailability relative to LPA might represent some of the causes for its higher efficacy in the apoptosis assays. The observation that OTP elicited ERK1/2 and AKT phosphorylation with longer duration in the mouse intestinal tissue certainly supports this hypothesis. At the same time, the lack of absorption of $\left[{ }^{3} \mathrm{H}\right]-\mathrm{OTP}$ into the systemic circulation within a 3 -h period, which appears to be the limit for the duration of its protective time window (data not shown), indicates that it exerts its effect topically from within the intestinal lumen without appearing in a pharmacologically and biologically effective concentration in the bloodstream.

Radioactively labeled LPA has been shown to be taken up rapidly (within minutes) into cells where it is rapidly dephosphorylated and reacylated (van der Bend,1992); nevertheless, $\sim 15 \%$ remains intact in the cytoplasm up to 30 min after extracellular application (Tokumura, 1992). These differences in bioavailability could, at least in part, explain the differences in the in vivo efficacy of OTP compared to LPA.

Another important difference was that the $\mathrm{LPA}_{2}$ receptor subtype is sufficient and necessary for the antiapoptotic effect of OTP. In the receptor reconstitution experiments carried out in RH7777 cells stably transfected with the individual receptor subtypes, although OTP activated $\mathrm{Ca}^{2+}$ transients through each receptor subtype, only cells expressing $\mathrm{LPA}_{2}$ were protected against apoptosis. In agreement with the role of $\mathrm{LPA}_{2}$ in this model system, experiments conducted in $\mathrm{LPA}_{2} \mathrm{KO}$ mice confirmed that this receptor was absolutely necessary for the attenuation of apoptosis and increased crypt survival elicited by OTP. A third line of evidence, namely the increased radiation sensitivity of $\mathrm{LPA}_{2} \mathrm{KO}$ gut tissue compared to wild type animals, suggests that this receptor subtype is uniquely involved in mediating prosurvival signals. Only if we assign the prosurvival function to $\mathrm{LPA}_{2}$ can it explain the lack of protective effect in response to LPA administration observed in the LPA $2 \mathrm{KO}$ mice, which continue to express $\mathrm{LPA}_{1}$ and $\mathrm{LPA}_{3}$ (Contos, 2002). RT-PCR analysis has identified only the LPA 1 and LPA 2 receptor 
subtypes in the mouse intestine, as well as in IEC-6 cells used in the present study ( $\mathrm{Li}$, 2005; Yun, 2005). The partial sensitivity of the OTP-elicited antiapoptotic response to PTX is consistent with the Gq-coupling of $\mathrm{LPA}_{2}$ (Ishii, 2000). However, LPA also showed antiapoptotic protection in RH7777 cells expressing either $\mathrm{LPA}_{1}$ or $\mathrm{LPA}_{3}$ in the TNF- $\alpha / C H X$ induced apoptosis model. The RH7777 cells show extreme resistance to radiation (Deng \& Tigyi unpublished) that precluded studying the radioprotective effect exerted by these receptors in this model. We do not understand the reasons for this difference and can only speculate that either the higher than physiological level of receptor over-expression or the differences in the protective signaling mechanism between the TNF- $\alpha / \mathrm{CHX}$ and the DNA damage-induced mechanisms could be part of the reason. We also found that caspase 8 activity was attenuated by OTP indicating that LPA receptor signaling affects the upstream part of the extrinsic apoptotic pathway. Whether this inhibition of caspase 8 activation is unique to $\mathrm{LPA}_{2}$ or also elicited by other receptor subtypes should be evaluated in future experiments. While this unanswered question does not detract from the importance of $\mathrm{LPA}_{2}$ in the radioprotective mechanisms activated by LPA or OTP and the natural radiation sensitivity of the gut tissue, further experiments will have to be designed to resolve this apparent contradiction. $\mathrm{LPA}_{2}$ is distinct from the other two subtypes in that its C-terminus has been shown to interact with PDZ and LIM domain-containing proteins (Oh, 2004; Xu, 2004; Lai, 2005; Li, 2005a; Yamada, 2005). $\mathrm{LPA}_{2}$ can signal through specific protein-protein interactions in a non-G-protein-coupled manner and access triple LIM domain-containing proteins including TRIP6, zyxin, LPP, and Siva-1 (Xu, 2004; Lai, 2005; Takizawa, 2006). G protein-linked activation of c-src by LPA 2 phosphorylates TRIP6, which in turn augments LPA-induced ERK activation (Lai, 2005) and could lead to increased BAD phosphorylation and inhibition of procaspase 9 (Fang, 1999). The potential interaction of $\mathrm{LPA}_{2}$ with Siva-1 offers an exciting possibility, because DNA damage-induced activation of Siva-1 scavenges the antiapoptotic Bcl-X $\mathrm{L}_{\mathrm{L}}$ (Xue, 2002; Chu, 2004; Gudi, 2006). LPA 2 could capture Siva-1 and enable Bcl- $\mathrm{X}_{\mathrm{L}}$ to attenuate apoptosis triggered by DNA damage. Our findings concerning the increased level of Bcl-XL in IEC-6 cells and OTP-treated gut favor this hypothesis. The PDZ domain-mediated interactions including the PDZ-binding protein NHERF2 provide yet another link with antiapoptotic signaling, because stable knockdown of NHERF2 in CaCo-2 cells has been found to attenuate $\mathrm{LPA}_{2}$-induced ERK1/2, AKT, and PLC $\beta$ activation (Oh, 2004; Yun, 2005). These potential signaling interactions between $\mathrm{LPA}_{2}$ and LIM or PDZ domain proteins await further investigation.

Orally administered OTP may offer other uses that reach beyond the protection of the GI system. Orally applied OTP is unlikely to be useful for reducing apoptosis in the hematopoietic system, because it does not get absorbed to a biologically effective concentration in the systemic circulation. However, experiments done with intraperitoneal application of OTP in irradiated C57BL/6 mice caused a significant reduction in lethality mitigating the lethality of the $\mathrm{LD}_{100 / 30}$ dose to equal that of a $\mathrm{LD}_{50 / 30}$ outcome. Thus, OTP's applicability for systemic treatment of radiation illness should be the focus of the next experiments.

In summary, the present study identifies OTP as an orally active antiapoptotic agent that targets $\mathrm{LPA}_{2}$ and distinct prosurvival signals coupled to this receptor. The 
simple chemical nature of OTP, combined with its metabolic resistance, limited systemic bioavailability, and its apparently low acute oral toxicity $(>10 \mathrm{mg} / \mathrm{kg}$ ) make this compound a reasonable candidate for treating radiation injury of the gut and warrant further investigation for its potential therapeutic uses. 


\section{CHAPTER 3. INTERACTION OF LPA2 WITH LIM AND PDZ DOMAIN PROTEINS MEDIATES ITS PROTECTION AGAINST APOPTOSIS}

\subsection{INTRODUCTION}

LPA is a phospholipid growth factor mediating diverse cellular responses via its cognate LPA receptors that belong to the G-protein coupled receptors (GPCRs). By coupling to $\mathrm{G}_{\mathrm{i}}, \mathrm{G}_{\mathrm{q}}, \mathrm{G}_{12 / 13}$ and $\mathrm{G}_{\mathrm{s}}$, LPA receptors mediate a vast array of biological and pathological responses including cell growth, survival, differentiation, migration and cancer invasion in response to LPA stimulation (Mills \& Moolenaar, 2003 Review). So far seven LPA receptors have been identified. $\mathrm{LPA}_{1}, \mathrm{LPA}_{2}$, and $\mathrm{LPA}_{3}$ belong to the EDG family and share more than 50\% homology (Anliker \& Chun, 2004 review). $\mathrm{LPA}_{4}$ (GPR23) and LPA 5 (GPR92) belong to a purinergic P2Y receptor subfamily (Noguchi, 2003; Kotarsky, 2006). Unlike $\mathrm{LPA}_{1-3}, \mathrm{LPA}_{4}$ and $\mathrm{LPA}_{5}$ mediate an increase in cAMP production (Noguchi, 2003; Kotarsky 2006; Lee, 2006). GPR87 (potential LPA6) and P2Y5 (potential $\mathrm{LPA}_{7}$ ), also belonging to the P2Y family, have recently been determined to be LPA receptors (Tabata, 2007; Shimomura, 2008). P2Y5 is found to be essential for maintenance of human scalp hair growth (Pasternack, 2008; Shimomura, 2008).

Substantial evidence has shown that LPA is a potent survival and antiapoptotic factor capable of arresting the apoptotic pathway in apoptotically condemned cells. LPA protects various cell lines from apoptosis induced by different apoptotic stimuli that include serum-deprived apoptosis in fibroblasts (Fang, 2000), macrophages (Koh, 1998), T cells (Goetzl, 2000), Schwann cells (Weiner, 1999; Li, 2003), renal proximal tubular cells (Levine, 1997), intestinal epithelial cells (Deng, 2002; 2007); chemotherapeutic agents-induced apoptosis in BJAB and I-83 B cell lines, primary chronic lymphocytic leukemia (CLL) cell lines (Hu, 2005), prostate cancer cells (Raj, 2004), intestinal epithelial cells (Deng, 2002; 2007), colon cancer cell lines (Rusovici, 2007), ovarian cancer cell lines (Frankel, 1996; Meng, 2005); death receptor-induced apoptosis (Kang, 2004; E, 2007), and radiation-induced apoptosis in epithelium (Deng, 2002; 2007). The protection against apoptosis by LPA is mainly mediated by LPA 1 and LPA 2 . ERK and/or Akt/PKB signaling pathways have been identified to mediate the LPA anti-apoptotic effects in most of these cell lines (Radeff-Huang, 2002 review). Previously we found OTP, an LPA mimic, protects epithelial cells from radiation-induced apoptosis both in vivo and in vitro (E, 2007). We also determined that only $\mathrm{LPA}_{2}$ reconstitution of $\mathrm{RH} 7777$ cells devoid of EDG family LPA receptors but not reconstitution with $\mathrm{LPA}_{1}$ or $\mathrm{LPA}_{3}$ was capable of rescuing these cells from TNF $\alpha$-induced apoptosis. In support of the essential role of $\mathrm{LPA}_{2}$, neither LPA nor OTP protected the intestinal mucosa of $\mathrm{LPA}_{2}$ knockout mice from radiation-induced apoptosis, whereas both ligands were active in $\mathrm{LPA}_{1}$ receptor knockouts. $\mathrm{LPA}_{2}$ is over-expressed in several cancer cell lines such as ovarian, colon and thyroid cancer cell lines (Zheng, 2000; Schulte, 2001; Fang, 2004; Shida, 2004; Rusovici, 2007), where it could contribute to the therapeutic resistance of these cancers by increasing their apoptotic resistance to radiation and chemotherapy. However, the molecular mechanisms of rescuing apoptotically challenged cells distinctive to $\mathrm{LPA}_{2}$ remain unknown. 
More and more evidence shows that GPCR can also relay signal through mechanisms distinct from the classical $\mathrm{G}$ protein-dependent signaling paradigm (Brady \& Limbird 2002 review; Hall \& Lefkowitz, 2002 review; Rajagopal, 2005 review; McGarrigle, 2007 review; Sun, 2007 review, Lefkowitz, 2007 review). These novel mechanisms involve signal transduction through macromolecular complex formation of GPCR by protein-protein interaction with adaptor or scaffold proteins. Examples of these adaptor proteins are $\beta$-arrestins (Lefkowitz, 2006) and PDZ domain containing proteins (Hung \& Sheng, 2002 review). AT1 receptor having mutations in the second intracellular DRY motif lacks $G$ protein coupling mediates activation of Src-Ras-ERK pathway (Seta, 2002). The conserved YIPP motif in the AT1 cayboxyl terminus interacts with SHP2, which in turn mediates Jak/STAT pathway and EGF receptor (Marrero, 1998; Seta, 2003). In addition, Sadoshima's group has provided the most compelling evidence using transgenic mice that $G$ protein-independent signal transdution via AT1R results in ventricular hypertrophy and bradycardia with a diminished apoptosis and a unique signaling profile (Zhai, 2005). Huang's group has demonstrated dose-dependent switch of G protein-coupled to $G$ protein-independent signaling by $\beta_{2}$ - ARs (Sun, 2007)

$\mathrm{LPA}_{1}, \mathrm{LPA}_{2}$, and LPA 3 share high, over $80 \%$, sequence homology in their transmembrane domains, whereas the carboxyl termini show a mere $11 \%$ homology. Intriguingly only the $\mathrm{CT}$ of $\mathrm{LPA}_{2}$ receptor, among the three EDG subtypes, can form protein-protein complexes with PDZ and LIM domain proteins (Lin, 2008 review). The identification of an increasing list of binding proteins unique to $\mathrm{LPA}_{2}-\mathrm{CT}$ warrants further study on the role of protein-protein interaction in mediating the function of $\mathrm{LPA}_{2}$ (Oh, 2004; Wang, 2004; Xu, 2004; Yamada, 2005; Lin, 2007; Zhang, 2007).

The PDZ domain is a modular protein-interaction domain that consists of around 90 amino acids. It is named after the three founding PDZ-containing proteins: the postsynaptic protein PSD-95/SAP90, the Drosophila septate junction protein Disc-large, and the tight junction protein $\mathrm{ZO}-1$. So far, at least four PDZ-containing proteins have been found to interact with $\mathrm{LPA}_{2}: \mathrm{Na}^{+} / \mathrm{H}^{+}$exchange regulatory factor 2 (NHERF2) (Oh, 2004; Yun, 2005), PDZ-RhoGEF (Yamada, 2005), leukemia-associated RhoGEF (Wang, 2004), and membrane associated guanylate kinase-like protein with an inverted domain structure-3 (MAGI-3) (Zhang, 2007). The best-studied PDZ domain protein interacting with $\mathrm{LPA}_{2}-\mathrm{CT}$ is NHERF2, which contains two tandem PDZ domains capable of bridging two proteins that contain PDZ motifs. The second PDZ domain of NHERF2 interacts specifically with $\mathrm{LPA}_{2}$ via its CT motif (D-S-T-L), but not with $\mathrm{LPA}_{1}$ or $\mathrm{LPA}_{3}$ (Oh, 2004; Li, 2005a). Knockdown of NHERF2 in Caco-2 cells attenuates $\mathrm{LPA}_{2}-$ mediated ERK1/2 and Akt activation (Oh, 2004; Yun, 2005). Moreover, the $\mathrm{LPA}_{2}$ receptor forms a macromolecular complex with Cystic Fibrosis Transduction Regulator (CFTR) and NHERF2 via the second PDZ domain (Li, 2005a). LPA attenuates cholera toxin-induced secretory diarrhea via formation of the protein-protein interaction complex between $\mathrm{LPA}_{2}$ and $\mathrm{CFTR} \mathrm{Cl}^{-}$channel (Li, 2005a). It is not clear whether association with these PDZ proteins mediates the anti-apoptotic function of $\mathrm{LPA}_{2}$.

Recently, the LIM domain proteins Thryoid Hormone Receptor Interacting Protein 6 (TRIP6) and the proapoptotic transcriptional coregulator Siva-1 have been 
found to interact with $\mathrm{LPA}_{2}$ by yeast two-hybrid system (Xu, 2004; Lin, 2007). The LIM domain is named by the acronym of three homeodomain proteins: Lin-11, Isl-1 and Mec3. It usually consists of approximately 55 amino acids with 8 highly conserved residues, mostly cysteine and histidine located at defined intervals, which constitute two sequential zinc-finger structures linked by a two-peptide linker (Fig 1.3) (Bach, 2000 review; Kadrmas \& Beckerle, 2004 review; Zheng \& Zhao, 2007 review). TRIP6 belongs to the LIM domain protein subfamily of Zyxin, which consists of Zyxin, LPP (lipoma preferred partner) and Ajuba (Beckerle, 1997 review; Yi \& Beckerle, 1998). The Zyxin family is associated with actin cytoskeleton reorganization Siva-1, though functionally unrelated to Zyxin family, shares structural similarity with TRIP6 in that it contains two carboxyl cysteine-rich zinc finger motifs and these cysteines are also present in the human and rat homologues (Prasad, 1997; Lin, 2007).

TRIP6 contains an N-terminal proline-rich domain, three LIM domains and a PDZ protein-binding motif at its CT (Yi, 1998; Xu, 2004). All three LIM domains are involved in interaction with $\mathrm{LPA}_{2}$. This association promotes LPA-dependent recruitment of TRIP6 to the focal adhesion where it interacts with focal adhesion molecules: focal adhesion kinase, $\mathrm{p} 130^{\mathrm{CAS}}$, paxillin and c-Src. Interestingly, c-Src can phosphorylate TRIP6 at Tyr-55 and this phosphorylation enhances the LPA-induced ERK1/2 activation. Knockdown of TRIP6 drastically attenuates LPA-induced ERK1/2 activation in c-Src expressing cells (Lai, 2005). Recently the same group has found that PTPL1/FAP-1 dephosphorylates TRIP6 at Tyr-55, which provides a negative regulation of the LPAinduced morphological change and cell migration (Lai, 2007). Furthermore, LPA activates Src (Luttrell, 1996) and inhibition of Src by the specific inhibitor PP2 abolishes its protective effect (Deng \& Tigyi - unpublished). TRIP6 also potentiates receptorinteracting protein 2 (RIP2)-mediated NF- $\mathrm{KB}$ and ERK activation ( $\mathrm{Li}, 2005 \mathrm{~b}$ ). These observations suggest that TRIP6 could be involved in mediating the anti-apoptotic effect of $\mathrm{LPA}_{2}$ by ERK activation, which is a required signal for the protective effect of LPA (Deng, 2003, E, 2007).

Siva-1 is a pro-apoptotic molecule initially discovered by using the cytoplasmic tail of CD27 as bait in a yeast two-hybrid system (Prasad, 1997). Siva-1 is not related to either death domain containing proteins like FADD or TNFR associated factors (TRAFs) domain proteins. Also, Siva-1 does not contain any Bcl-2 Homology (BH) domains. So it is not related to Bcl-2 family members. Over-expression of Siva-1 in various cell lines induced apoptosis (Prasad, 1997). Siva-1 expression is regulated by p53 and E2F1 (Fortin, 2004) and is up regulated during DNA damage responses (Qin, 2002; Daoud, 2003; Fortin, 2004: Lin, 2007). Interestingly, Siva-1 also binds to Bcl- $\mathrm{X}_{\mathrm{L}}$ and inhibits $\mathrm{Bcl}-\mathrm{X}_{\mathrm{L}}$ mediated protection against UV radiation-induced apoptosis (Xue, 2002). A putative amphipathic helical region of Siva-1 is found to be sufficient to bind Bcl- $\mathrm{X}_{\mathrm{L}}$ and sensitize cells to UV radiation-induced apoptosis (Chu, 2004). The underlying mechanism of apoptosis promotion of Siva-1 involves cytochrome $\mathrm{C}$ release and activation of caspases 9 and 3. In T lymphocytes, overexpression of Siva proteins induces apoptosis via a caspase-dependent mitochondrial pathway (Py, 2004). Most interestingly, Siva-1 is found to interact $\mathrm{LPA}_{2}-\mathrm{CT}$ (Lin, 2007). The promoting effect of Siva-1 in DNA damage-induced apoptosis is inhibited by LPA pretreatment (Lin, 2007). $\mathrm{LPA}_{2}$ forms a 
macromolecular complex with Siva-1 and this complex becomes polyubiquitinated in response to LPA stimulation of cells and in turn the complex is degraded in the proteasome. Knockdown of LPA $_{2}$ expression increases Siva-1 protein levels and augments adriamycin-induced caspase-3 activation and apoptosis (Lin, 2007). Perhaps not just a coincidence that LPA up regulates Bcl- $\mathrm{X}_{\mathrm{L}}$ levels at the posttranscriptional level in culture intestinal epithelial cells and also in the gut epithelium of mice treated with OTP (E, 2007). This coincidence provides the foundation for our hypothesis that interaction of Siva-1 with $\mathrm{LPA}_{2}$ is involved in the antiapoptotic rescue mechanism. However, it is not clear how $\mathrm{LPA}_{2}$ interacts with Siva-1 and attenuates its proapoptotic function.

In this work we have mapped out the motif in $\mathrm{LPA}_{2}-\mathrm{CT}$ that mediates interaction with TRIP6 and Siva-1 and examined LPA 2 downstream signaling involved in apoptosis protection via disruption of its interaction with LIM and/or PDZ domain proteins.

\subsection{MATERIALS AND METHODS}

\subsubsection{Materials}

LPA (18:1) was purchased from Avanti Polar Lipids (Alabaster, AL). Fura-2/AM, Alexa fluor 488 donkey anti-mouse IgG conjugate, TRIzol, DNase I and ThermoScript RT-PCR System for First-Strand cDNA Synthesis were obtained from Invitrogen (Carlsbad, CA). Bovine serum albumin (BSA; fraction V, fatty acid-free), mouse monoclonal and rabbit polyclonal anti-Flag M2 antibody and affinity gel, normal donkey serum, and 2-bromopalmitate were from Sigma. Rabbit anti-phospho-anti-ERK1, rabbit anti-phospho-AKT, rabbit anti-AKT IgG were from Cell Signaling Biotechnology (Worcester, MA). Anti-Mouse and anti-rabbit IgG HRP conjugate were from Promega (Madison, WI). Plasmid extraction kit was from Qiagen (Valencia, CA). Restriction enzymes were from New England Biolab (Beverly, MA). RT2 Real-Time SYBR Green/ROX was from SuperArray (Frederick, MD). Re-Blot plus strong solution (10X) was from Chemicon (Temecula, CA). Adriamycin was from Teva Pharmaceuticals (Pennsylvania).

\subsubsection{Plasmid Construction and Site-directed Mutagenesis}

The wild type (WT) N-terminal FLAG epitope-tagged human (h)LPA 2 receptor construct subcloned into the pcDNA3.1 expression plasmid was provided by Dr. Junken Aoki (University of Tokyo, Tokyo, Japan). Truncation mutations were generated using T7 primer: 5'-TAA TAC GAC TCA CTA TAG GG-3' and the corresponding reverse primer designed to contain the PDZ binding motif DSTL and Xbal restriction sites (Table 3.1.). The PCR product was digested with EcoR I and Xba 1, subcloned into pcDNA3.1. Alanine mutants were generated overlapping extension PCR with primers containing sitespecific mutations (Table 3.2.) and T7 (see above) and BGH reverse primer (5'- 
Table 3.1. Primer sequences for hLPA $A_{2}$ truncation mutants.

\begin{tabular}{cc}
\hline $\begin{array}{c}\text { Truncation } \\
\text { mutation }\end{array}$ & Primer sequence \\
\hline $\begin{array}{c}\Delta 302+ \\
\text { DSTL }\end{array}$ & $5^{\prime}$ - GCT CTA GAC TAC AGA GTT GAA TCC ATC TCA GCA TCT CGG CAA G - 3' \\
$\Delta 309+$ & $5^{\prime}$ - GCT CTA GAC TAC AGA GTT GAA TCA AGG CGG CGG AAG GTG CG - 3' \\
DSTL & \\
$\Delta 316+$ & $5^{\prime}$ - GCT CTA GAC TAC AGA GTT GAA TCG CGG AGG CAC GCG CAG CA - 3 \\
DSTL & \\
$\Delta 327+$ & $5^{\prime}$ - GCT CTA GAC TAC AGA GTT GAA TCG GAT GTA TAG TGG ACA GAC TC- 3' \\
DSTL & \\
$\Delta 338+$ & $5^{\prime}$ - GCT CTA GAC TAC AGA GTT GAA TCC ATG ATG CGA GTG CTG GCA CC-3' \\
DSTL & \\
$\Delta 347$ & $5^{\prime}-$ GCT CTA GAC TAC ATC AGT GGG TGG CCG TTC TC - 3' \\
\hline
\end{tabular}


Table 3.2. Primer sequences for $h \mathrm{LPA}_{2}$ alanine point mutants.

\begin{tabular}{|c|c|c|c|}
\hline Mutation & Codon & Mutated codon & Primer sequence \\
\hline C297A & TGC & GCT & $\begin{array}{l}\text { F:5'GTGTACTCTGCTCGAGATGCT3' } \\
\text { R:5'AGCATCTCGAGCAGAGTACAC3', }\end{array}$ \\
\hline R298A & CGA & GCA & $\begin{array}{l}\text { F:5'GCTGCTGTGTACTCTTGCGCAGATGCTGAGATGC3' } \\
\text { R:5'GCATCTCAGCATCTGCGCAAGAGTACACAGCAGC3', }\end{array}$ \\
\hline D299A & GAT & GCT & $\begin{array}{l}\text { F:5'TCTTGCCGAGCTGCTGAGATG3' } \\
\text { R:5'CATCTCAGCAGCTCGGCAAGA3' }\end{array}$ \\
\hline E301A & GAG & GCT & $\begin{array}{l}\text { F:5'CTCTTGCCGAGATGCTGCTATGCGCCGCACC3' } \\
\text { R:5'GGTGCGGCGCATAGCAGCATCTCGGCAAGAG }\end{array}$ \\
\hline R303A & CGC & GCT & $\begin{array}{l}\text { F:5'CTCTTGCCGAGATGCTGAGATGGCTCGCACCTTCC3', } \\
\text { R:5'GGAAGGTGCGAGCCATCTCAGCATCTCGGCAAGAG3' }\end{array}$ \\
\hline R304A & CGC & GCT & $\begin{array}{l}\text { F:5'TGAGATGCGCGCTACCTTCCGC3' } \\
\text { R:5'GCGGAAGGTAGCGCGCATCTCA3', }\end{array}$ \\
\hline $\mathrm{T} 305 \mathrm{~A}$ & $\mathrm{ACC}$ & GCT & $\begin{array}{l}\text { F:5'GAGATGCGCCGCGCTTTCCGCCGCCTTC3' } \\
\text { R:5'GAAGGCGGCGGAAAGCGCGGCGCATCTC3', }\end{array}$ \\
\hline F306A & TTC & GCT & $\begin{array}{l}\text { F:5'CCGCACCGCTCGCCGCCTTCT3' } \\
\text { R:5'AGAAGGCGGCGAGCGGTGCGG3', }\end{array}$ \\
\hline R307A & CGC & GCT & $\begin{array}{l}\text { F:5'GATGCGCCGCACCTTCGCTCGCCTTCTCTGCTG3' } \\
\text { R:5'CAGCAGAGAAGGCGAGCGAAGGTGCGGCGCATC3' }\end{array}$ \\
\hline R308A & CGC & GCT & $\begin{array}{l}\text { F:5'ACCTTCCGCGCTCTTCTCTGC3' } \\
\text { R:5'GCAGAGAAGAGCGCGGAAGGT3' }\end{array}$ \\
\hline L309A & CTT & GCT & $\begin{array}{l}\text { F:5'TTCCGCCGCGCTCTCTGCTGC3', } \\
\text { R:5'GCAGCAGAGAGCGCGGCGGAA3' }\end{array}$ \\
\hline
\end{tabular}


Table 3.2. (Continued).

\begin{tabular}{|c|c|c|c|}
\hline Mutation & Codon & Mutated codon & Primer sequence \\
\hline $\mathrm{C} 312 \mathrm{~A}$ & TGC & GCT & $\begin{array}{l}\text { F:5'CTTCTCTGCGCTGCGTGCCTC3' } \\
\text { R:5'GAGGCACGCAGCGCAGAGAAG3' }\end{array}$ \\
\hline R316A & CGC & GCT & $\begin{array}{l}\text { F:5'CTGCGCGTGCCTCGCTCAGTCCACCCGCGAG3', } \\
\text { R:5'CTCGCGGGTGGACTGAGCGAGGCACGCGCAG3' }\end{array}$ \\
\hline C314A/L351A & $\mathrm{TGC} / \mathrm{CTT}$ & GCT/GCT & $\begin{array}{l}\text { F: 5'TCTGCTGCGCGGCTCTCCGCCAGT3' } \\
\text { R: 5'ACTGGCGGAGAGCCGCGCAGCAGA3', }\end{array}$ \\
\hline C314G/L351A & $\mathrm{TGC} / \mathrm{CTT}$ & GGT/GCT & $\begin{array}{l}\text { F:5'TCTGCTGCGCGGGTCTCCGCCAGT3', } \\
\text { R:5'ACTGGCGGAGACCCGCGCAGCAGA3' }\end{array}$ \\
\hline $\mathrm{C} 311 \mathrm{~A} / \mathrm{C} 314 \mathrm{~A}$ & TGC/TGC & GCT/GCT & $\begin{array}{l}\text { F:5'CGCCTTCTCGCTTGCGCGTGC3' } \\
\text { R:5'GCACGCGCAAGCGAGAAGGCG3' }\end{array}$ \\
\hline $\mathrm{C} 311 \mathrm{~A} / \mathrm{C} 314 \mathrm{~A} / \mathrm{L} 351 \mathrm{~A}$ & TGC/TGC/CTT & GCT/GCT/GCT & $\begin{array}{l}\text { F:5'CGCCTTCTCGCTTGCGCGTGC3' } \\
\text { R:5'GCACGCGCAAGCGAGAAGGCG3' }\end{array}$ \\
\hline $\mathrm{C} 311 \mathrm{~A} / \mathrm{C} 312 \mathrm{~A} / \mathrm{C} 314 \mathrm{~A}$ & TGC/TGC/TGC & GCT/GCT/GCT & $\begin{array}{l}\text { F:5'CGCCTTCTCGCTGCTGCGGCTCTCCGCCAG3' } \\
\text { R:5'CTGGCGGAGAGCCGCAGCGAGAAGGCG3' }\end{array}$ \\
\hline
\end{tabular}


TAGAAGGCACAGTCGAGG). hLPA 2 WT and the generated mutants were digested with EcoR I and Not I and subcloned into pcDNA3.1/Zeocin (+) vector (Invitrogen). Mutated DNA was transformed into TOP10-competent cells (Invitrogen), and clones were verified by complete sequencing of the inserts. The L351A mutant was a kind gift from Dr. A.P. Naren (Li, 2005a).

\subsubsection{Cell Culture and Transfection}

EDG-family LPA receptor null RH7777 (RH) cells (ATCC, Manassas, VA) were maintained in Dulbecco's modified Eagle's medium (DMEM) supplemented with 10\% fetal bovine serum (Hyclone, Logan, UT) and antibiotics (Invitrogen). Cells were transfected using Effectene according to manufacturer's protocol (Qiagen).

Primary Mouse Embryonic Fibroblasts (MEFs) were isolated from E13.5 $\mathrm{LPA}_{1 / 2}$ Double knockout (DKO) embryos (Yue, 2004) and maintained in DMEM supplemented with $10 \%$ fetal bovine serum and antibiotics. Primary MEFs were immortalized by transfection with SV40 large T antigen or by selecting spontaneously immortalized cells. Genotype of $\mathrm{LPA}_{1 / 2}$ DKO MEFs was confirmed as described previously (Contos, 2002).

\subsubsection{Construction of LPA $A_{2}$-Lentiviral Vectors and Production of Lentivirus}

pcDNA3.1-hLPA 2 WT and mutants were digested with XhoI and filled in with DNA polymerase Klenow, then re-digested with NheI and the cDNA fragment was purified. The lentiviral vector pcFUW-puro was excised with BamHI and filled in with Klenow, then re-excised with Xba I. The $\mathrm{LPA}_{2}$ wild type and mutant cDNA fragments were purified and subcloned into Xba I and blunted BamH I sites of pcFUW-puro. The recombinant lentivirus was produced in 293FT cells (Invitrogen) by co-transfection of the lentiviral transfer vector with the ViraPower Packaging Mix (Invitrogen) containing plasmids, pLP/VSVG, pLP1/gag-pol, and pLP2-Rev. The supernatant was collected $60 \mathrm{~h}$ after transfection and centrifuged $15 \mathrm{~min}$ at $3000 \mathrm{x}$ g at $4{ }^{\circ} \mathrm{C}$ to remove cell debris, then the supernatant was passed through $0.45 \mu \mathrm{m}$ filter and collected for ultra-centrifugation at $54000 \mathrm{x}$ g for $3 \mathrm{~h}$ at $4{ }^{\circ} \mathrm{C}$. The virus pellet was dissolved in PBS and stored at $-80^{\circ} \mathrm{C}$. The viral particles were titrated in 293 FT cells using p24 ELISA assay (PerkinElmer, Boston, MA). Stable MEFs expressing GFP, empty vector, WT $\mathrm{LPA}_{2}$ and the different $\mathrm{LPA}_{2}$ mutants were derived by transduction with lentiviruses and selected with $1.5 \mu \mathrm{g} / \mathrm{ml}$ puromycin.

\subsubsection{Ligand-induced $\mathrm{Ca}^{2+}$ Activation Assay}

RH7777 cells plated in 6-well plates were transiently transfected with either $2 \mu \mathrm{g}$ of WT LPA ${ }_{2}$ or its mutants and cultured for $24 \mathrm{~h}$ and replated onto $0.1 \mathrm{mg} / \mathrm{ml}$ poly-Llysine-coated 96-well microplates (Corning Incorporated Life Sciences, Acton, MA) at 3 x $10^{4}$ cells/well. Lentivirus-transduced $\mathrm{LPA}_{1}$ and $\mathrm{LPA}_{2}$ double knockout MEFs stably 
expressing WT LPA 2 and its mutants, $\mathrm{LPA}_{1} \mathrm{KO}$ MEFs (express endogenous level of $\mathrm{LPA}_{2}$ ) were plated onto poly-L-lysine-coated 96-well microplates at a density of $4 \times 10^{4}$ cells/well and cultured overnight. RH cells after $5 \mathrm{~h}$ serum starvation or MEFs with no serum starvation were loaded with Fura-2/AM in Krebs buffer $(120 \mathrm{mM} \mathrm{NaCl}, 5 \mathrm{mM}$ $\mathrm{KCl}, 0.62 \mathrm{mM} \mathrm{MgSO}_{4}, 1.8 \mathrm{mM} \mathrm{CaCl}_{2}, 10 \mathrm{mM}$ HEPES, pH 7.4, $6 \mathrm{mM}$ glucose) containing $2 \%(\mathrm{v} / \mathrm{v})$ pluronic acid at $37{ }^{\circ} \mathrm{C}$ for $30 \mathrm{~min}$. The cells were rinsed with Krebs buffer and monitored in a FLEXStation (Molecular Devices, Sunnyvale, CA) at excitation wavelengths of $340 / 380 \mathrm{~nm}$ and an emission wavelength of $510 \mathrm{~nm}$ for $70 \mathrm{sec}$ after the addition of different concentration of LPA 18:1 or $10 \mu \mathrm{M}$ ATP (positive control).

\subsubsection{Western Blot}

ERK1/2 and Akt were measured in total cellular protein lysates by using a previously described procedure (E, 2007). Briefly, lentivirus-transduced stable mock, WT $\mathrm{LPA}_{2}$ MEFs and those expressing the LPA 2 mutants were plated at $80 \%$ confluence and cultured overnight. On the second day, cells were serum starved for $2.5 \mathrm{~h}$ and treated with $1 \mu \mathrm{M}$ LPA for indicated times. Cells were lysed in $62.5 \mathrm{mM}$ Tris-HCl (pH 6.8), $2 \%$ SDS, $25 \%$ glycerol plus phosphatase and protease inhibitor cocktail (Sigma). The lysates were sonicated twice for $5 \mathrm{~s}$, cleared by centrifugation at $1.6 \times 10^{4} \mathrm{~g}$ for $15 \mathrm{~min}$ at $4{ }^{\circ} \mathrm{C}$, and the supernatants were collected. Protein concentrations were determined using the BCA reagent kit (Pierce Biotechnology, Inc., Rockford, IL). For ERK1/2 $15 \mu \mathrm{g}$, Akt $80 \mu \mathrm{g}$ of cell lysate was fractionated by SDS-PAGE and transferred to PVDF membranes, blocked with $5 \%$ nonfat milk, and incubated with various primary antibodies. Blots were reacted with the appropriate HRP-conjugated secondary antibodies and developed using the SuperSignal chemiluminescence reagent (Pierce Biotechnology, Inc). Images were visualized with Fotodyne image analysis system (Fotodyne Inc., Hartland, WI).

\subsubsection{Reverse-transcription PCR}

Total RNA was extracted from spontaneously immortalized $\mathrm{LPA}_{1}$ and $\mathrm{LPA}_{2}$ double knockout MEFs stably expressing WT LPA 2, LPA $_{2}-\mathrm{C} 311 \mathrm{~A} / \mathrm{C} 314 \mathrm{~A}, \mathrm{LPA}_{2}-\mathrm{L} 351 \mathrm{~A}$ or $\mathrm{LPA}_{2}-\mathrm{C} 311 \mathrm{~A} / \mathrm{C} 314 \mathrm{~A} / \mathrm{L} 351 \mathrm{~A}$ or GFP using TRIzol reagent. Two $\mu \mathrm{g}$ total RNA was digested by DNase I. $400 \mathrm{ng}$ of the digest was subjected to reverse transcription PCR using the Superscript III kit to amplify $\mathrm{LPA}_{2}$. Primers sets were: forward, 5'CACTCCTGGCACTGCCTCTGTG-3', reverse, 5'-TACGGCGCATCTCAGCGTCTC G-3'. $\beta$-actin, forward, 5'-GACAACGGCTCCGGCATGTG-3', reverse, 5' -

TTGAGACCTTCAACACCCCAGCA -3'. A total of 31 amplification cycles were performed and the products were separated in $2 \%$ agarose gels, stained with ethidium bromide for visualization. 


\subsubsection{Quantitative Real Time-PCR}

Total RNA was prepared from spontaneously immortalized $\mathrm{LPA}_{1}$ and $\mathrm{LPA}_{2}$ double knockout MEFs stably expressing WT LPA $2, \mathrm{LPA}_{2}-\mathrm{C} 311 \mathrm{~A} / \mathrm{C} 314 \mathrm{~A}, \mathrm{LPA}_{2}-\mathrm{L} 351 \mathrm{~A}$ or $\mathrm{LPA}_{2}-\mathrm{C} 311 \mathrm{~A} / \mathrm{C} 314 \mathrm{~A} / \mathrm{L} 351 \mathrm{~A}$ or GFP using TRIzol. One $\mu \mathrm{g}$ of total RNA was digested with DNase I and used for the subsequent synthesis of cDNA using the First Strand Synthesis kit as recommended by the manufacturer. The following primer pairs were used: $\mathrm{LPA}_{1}$ : (forward) GTCTTCTGGGCCATTTTCAA and (reverse) TCATAGTCCTCTGGCGAACA; LPA 2 : (forward) GGGCCAGTGCTACTACAACG and (reverse) ACCAGCAGATTGGTCAGCA; LPA 3 : (forward) GAAGCTAATGAAGACGGTGATGA and (reverse) AGCAGGAACCACCTTTTCAC; LPA $_{4}$ : (forward) TCTGGATCCTAGTCCTCAGTGG and (reverse) CCAGACACGTTTGGAGAAGC; LPA : (forward) CGCCATCTTCCAGATGAAC and (reverse) TAGCGGTCCACGTTGATG; LPA6( GPR87): (forward) AAATCCAGCAGGCAATTCAT and (reverse) CCCTGATGCTCTGGTTATGTT ; $\mathrm{LPA}_{7}$ (P2ry5): (forward) TCTGGCAATTGTCTACCCATT and (reverse) TCAAAGCAGGCTTCTGAGG; $\beta$-actin: (forward) TTCTACAATGAGCTGCGTGTG and (reverse) GGGGTGTTGAAGGTCTCAAA. The primer sets were designed with melting temperatures in the $59-61{ }^{\circ} \mathrm{C}$ range and the amplicon size was 50-200 bp. Amplification was performed for 40 cycles at $94^{\circ} \mathrm{C} / 15 \mathrm{sec}$ and $60^{\circ} \mathrm{C} / 60 \mathrm{sec}$. Quantitative values of the threshold cycle value $(\mathrm{Ct})$, were determined using an $\mathrm{ABI}-$ Prism (manufacturer, city state) Q-PCR Instrument. The transcript number of (human/mouse/rat) $\beta$-actin was quantified to serve as internal RNA control, and each sample was normalized on the basis of its $\beta$-actin content. The relative expression level of each gene was then normalized to the calibrator. Final results, expressed as fold difference in gene expression relative to $\beta$-actin and gene of interest, termed $\mathrm{N}$, were calculated as: $\mathrm{N}=2^{\text {(Ct gene - Ct calibrator) }}$ (http://dorakmt.tripod.com/genetics/realtime.html), where $\mathrm{Ct}$ values of the gene and calibrator were determined by subtracting the average $\mathrm{Ct}$ value of a target gene from the corresponding $\mathrm{Ct}$ value of the $\beta$-actin gene.

\subsubsection{Flow Cytometry Analysis}

RH cells plated in 6-well plates were transfected with $0.4 \mu \mathrm{g}$ of DNA using Effectene. After $24 \mathrm{~h}$ incubation, the cells were serum starved for $6 \mathrm{~h}$ and trypsinized with HyQtase (Hyclone), washed twice with ice-cold FACS buffer consisting of phosphatebuffered saline (PBS) supplemented with 3\% (w/v) BSA, incubated in blocking buffer (PBS, 5\% BSA, 5\% normal donkey serum) for 30 min, washed with FACS buffer three times and incubated with monoclonal antibody in blocking buffer (1:100 dilution) for $1 \mathrm{~h}$. The anti-FLAG-stained cells were rinsed in FACS buffer three times, and incubated in the dark at $4{ }^{\circ} \mathrm{C}$ with AlexaFluor 488-conjugated donkey anti-mouse $\operatorname{IgG}$ at 1:1600 dilution for $30 \mathrm{~min}$. The cells were rinsed three times with FACS buffer and analyzed using an LSR II flowcytometer (Becton Dickinson, San Jose, CA). 


\subsubsection{Cellular Co-immunoprecipitation and GST Pull-down}

Cellularco-immunoprecipitation of Siva-1 or TRIP6 with the LPA receptors and the truncation and alanine point mutant swas performed as described previously $\mathrm{Xu}$, 2004). Briefly, HEK 293T cells transiently expressing MYC-TRIP6 with $\mathrm{LPA}_{2}$, or its mutants were starved in $0.1 \%$ fatty acid-free BSA containing DMEM for $8 \mathrm{~h}$ to overnight followed by the stimulation with $2 \mu \mathrm{M}$ LPA for $10 \mathrm{~min}$. The $\mathrm{LPA}_{2}$ was immunoprecipitated with the anti-FLAG M2 mouse monoclonal antibody-conjugated agarose beads (Sigma), resolved by SDS-PAGE, and the immunoblot was probed with an anti-MYC rabbit polyclonal antibody (Santa Cruz Biotechnology, Santa Cruz, CA) to detect the co immunoprecipitated MYC-TRIP6. The blots were stripped and reprobed with an anti-FLAG rabbit polyclonal antibody (Sigma) to detect the FLAG-LPA 2 receptor. To detect the cellular interaction of $\mathrm{LPA}_{2}$ with Siva-1, MYC-Siva-1 was coexpressed with $\mathrm{LPA}_{2}$ in HEK $293 \mathrm{~T}$ cells. After starvation overnight, cells were harvested and the co-immunoprecipitation was performed as described above. MYC-Siva-1 coimmunoprecipiated with FLAG-LPA 2 was detected with an anti-MYC rabbit polyclonal antibody.

To detect direct binding of Siva-1 or TRIP6 with the carboxyl-terminal tail of the $\mathrm{LPA}_{2}$ receptor, GST, GST-Siva-1, GST-TRIP6, GST-LPA 2 -CT or its alanine mutants were expressed in (BL21)(DE3)(LysS) E. coli and purified by immobilizing the proteins on glutathione-Sepharose 4B beads (Amersham Biosciences, Piscataway, NJ). GST-Siva1 and GST- TRIP6 was further digested with PreScission Protease (Amersham Bioscience) to cleave off the GST tag. $1 \mu \mathrm{g}$ of TRIP6 or $0.1 \mu \mathrm{g}$ of Siva-1 protein was incubated with $1 \mu \mathrm{g}$ of GST, GST-LPA 2 -CT or its mutants in the binding buffer $(20 \mathrm{mM}$ Tris, pH7.4, $1 \mathrm{mM}$ EDTA, $150 \mathrm{mM} \mathrm{NaCl}, 0.5 \%$ Nonidet P-40) for $3 \mathrm{~h}$ at $4{ }^{\circ} \mathrm{C}$. Siva-1 or TRIP6 protein pulled down by GST-LPA 2 -CT or alanine mutants was detected with an anti-Siva-1 polyclonal antibody (affinity purified anti-Siva-1 polyclonal antibody raised against human Siva-1 peptide aa30-44, Lin, 2007) and TRIP6 specific anitibody (Transduction Laboratory, San Jose, CA), respectively.

\subsubsection{Immunocytochemistry}

To examine co-localization of Siva-1 with the $\mathrm{LPA}_{2}$ receptor, GFP-Siva-1 was coexpressed with the FLAG-LPA ${ }_{2}$ or its mutants in SV40 T-antigen-transformed $\mathrm{LPA}_{1}$ and $\mathrm{LPA}_{2}$ double knockout MEFs. Cells were starved overnight in 0.1\% BSA-containing medium. After fixation, permeabilization and blocking, cells were incubated with an anti-FLAG M2 monoclonal antibody followed by a Texas Red X-conjugated anti-mouse secondary antibody (Invitrogen) to detect FLAG-LPA 2 constructs. The nuclei were counter stained with Hoechst 33258 (Sigma). The images of GFP and GFP-Siva-1 were captured by fluorescence microscopy using a Zeiss Axioplan 2 microscope. 


\subsubsection{Palmitoylation}

HEK 293 T cells were transfected with the plasmids indicated. Cells were labeled with $\left[{ }^{3} \mathrm{H}\right]$ palmitic acid at $37^{\circ} \mathrm{C}$ for $2 \mathrm{~h}$. The FLAG-LPA 2 receptor was immunoprecipitated with an anti-FLAG M2 monoclonal antibody-conjugated agarose beads, resolved by SDS-PAGE and detected by autoradiography and by immunoblotting with an anti-FLAG antibody, respectively.

\subsubsection{Acyl-Biotinyl Exchange Method}

GFP-Siva-1 or GFP-TRIP6 was co-expressed with the FLAG-LPA 2 receptor in HEK 293T cells. After treatment with $100 \mu \mathrm{M}$ 2-BP in $0.1 \%$ BSA-containing DMEM for $4 \mathrm{~h}$, cells were stimulated with LPA for $10 \mathrm{~min}$, and the FLAG-LPA 2 receptor was immunoprecipitated with the anti-FLAG antibody-conjugated agarose beads. Half of the precipitates were resolved by SDS-PAGE and the immunoblot was probed with an antiGFP polyclonal antibody to detect GFP-Siva-1 and GFP-TRIP6, respectively. The rest of the samples were subjected to acyl-biotinyl exchange (ABE) chemistry to determine the levels of palmitoylated $\mathrm{LPA}_{2}$ receptor (Roth, 2006a). Briefly, the precipitated $\mathrm{LPA}_{2}$ receptor was pre-treated with N-ethylmaleimide (NEM) followed by the acyl-biotinyl exchange with hydroxylamine and biotin-HPDP. The biotinylated FLAG-LPA 2 receptor was eluted from the beads using excess FLAG peptides for competition, and then precipitated with avidin beads. After SDS-PAGE, the immunoblot was probed with an anti-FLAG antibody to detect the palmitoylated FLAG-LPA 2 receptor.

\subsubsection{Adriamycin-induced Siva-1 Expression, Procaspase-3 Cleavage and Apoptosis}

The spontaneously immortalized $\mathrm{LPA}_{1}$ and $\mathrm{LPA}_{2}$ double knockout MEFs stably expressing WT LPA $2, \mathrm{LPA}_{2}-\mathrm{C} 311 \mathrm{~A} / \mathrm{C} 314 \mathrm{~A}, \mathrm{LPA}_{2}-\mathrm{L} 351 \mathrm{~A}$ or $\mathrm{LPA}_{2}-$ $\mathrm{C} 311 \mathrm{~A} / \mathrm{C} 314 \mathrm{~A} / \mathrm{L} 351 \mathrm{~A}$ were incubated with $10 \mu \mathrm{M}$ LPA in $0.1 \%$ BSA-containing DMEM for $2 \mathrm{~h}$ followed by the addition of $1.5 \mu \mathrm{M}$ adriamycin for $14 \mathrm{~h}$ and harvested. Halves of the samples were subjected to FITC-annexin V staining (BD Biosciences), and the apoptosis was determined by flow cytometry. The other halves of the samples were subjected to immunoblotting. The expression of procaspase-3 and Siva-1 in the total lysates was detected with a rabbit polyclonal antibody specific to caspase-3 (Santa Cruz Biotechnology) and Siva-1, respectively.

\subsubsection{DNA Fragmentation Assay}

DNA fragmentation was measured by ELISA following the procedure provided with the Cell Death Detection kit from Roche, Inc. (Indianapolis, IN) as described previously (Deng $2002 \& 2003$ ) and was expressed as absorbance units at $405 \mathrm{~nm}$ per microgram protein per minute. Briefly, the spontaneously immortalized LPA ${ }_{1}$ and LPA double knockout MEFs stably expressing WT LPA ${ }_{2}$, or its mutants were plated the night 
before the assay, at a density of $0.11 \times 10^{6}$ per well on 24 -well plates coated with 0.1 $\mathrm{mg} / \mathrm{ml}$ poly-L-lysine. On the second morning, cells were rinsed twice with DMEM containing $0.5 \% \mathrm{FBS}$ and treated with $3 \mu \mathrm{M}$ adriamycin for $6 \mathrm{~h} .2 \mu \mathrm{M}$ LPA was added $1 \mathrm{~h}$ after adriamycin treatment. The duplicate plate was used for protein assay with the BCA kit.

\subsubsection{Statistical Analysis of Data}

Data are expressed as means $\pm \mathrm{SD} / \mathrm{SEM}$. Each experiment was repeated at least three times. Student $t$ test was used for comparing different groups. A $p$ value of $<.05$ was considered significant.

\subsection{RESULTS}

\subsubsection{Spaced Cysteine Residues in $\mathrm{LPA}_{2}-\mathrm{CT}$ May Constitute Potential Zinc-finger Binding Structure to TRIP6/Siva-1}

Previously it has been shown that aa 296-351 of $\mathrm{LPA}_{2}$ is required for interaction with TRIP6 and Siva-1, but not $\mathrm{LPA}_{1}$ or $\mathrm{LPA}_{3}$ (Xu 2004; Lin, 2007). TRIP6 possesses 3 LIM domains at its CT (aa 278-476) and all of them contribute to the binding of LPA2. While Siva-1 has 2 zinc finger motifs at its CT (aa114-138 and aa142-170), only the second (aa 142-170) interacts with $\mathrm{LPA}_{2}$. Comparison of amino acid sequences between the second zinc finger domain of Siva-1 and TRIP6-LIM3 reveals conserved cysteine residues at defined intervals, which is the characteristic feature of LIM domains (Fig. 3.1 \& Fig. 1.4). $\mathrm{LPA}_{2}$ is distinctly different from the other receptor subtypes in its Cterminal tail (Fig. 3.1). Whereas the homology between $\mathrm{LPA}_{1}, \mathrm{LPA}_{2}$, and $\mathrm{LPA}_{3}$ in the transmembrane regions is $81 \%$, in the $\mathrm{C}$-terminal tail there are only 6 conserved amino acids in $55(11 \%)$. Among the 21 residues in CTs of $\mathrm{LPA}_{1}, \mathrm{LPA}_{2}$ and $\mathrm{LPA}_{3}$ that were aligned with zinc finger structure of TRIP and Siva-1, there are three conserved, spaced

\begin{tabular}{|c|c|c|c|}
\hline Human & Siva-1 & 142 & CWGCGSVACTLCGLVDCSDMYEKVLCTSCAM \\
\hline Human & TRIP6 & 432 & CEECGLLLSSEGECQGCYPLDGHILCKACSA \\
\hline Human & $\mathrm{LPA}_{2}-\mathrm{CT}$ & 296 & SCRDAEMRRTFRRLLCCACLR \\
\hline Human & $\mathrm{LPA}_{1}-\mathrm{CT}$ & 312 & SYRDKEMSATFRQILCCQRSE \\
\hline Human & $\mathrm{LPA}_{3}-\mathrm{CT}$ & 200 & đYGTMKKI \\
\hline
\end{tabular}

Figure 3.1. Amino acid sequence alignment of human Siva-1, TRIP6 and LPA receptor-CTs. 
cysteine residues in $\mathrm{LPA}_{2}$ compared to one in $\mathrm{LPA}_{1}$ and $\mathrm{LPA}_{3}$ (Fig. 3.1). Since zincfinger mediates protein-protein interaction, we hypothesize that these cysteine residues may constitute the binding motif of $\mathrm{LPA}_{2}$ to TRIP and/or Siva-1.

\subsubsection{TRIP6 and Siva-1 Binding Motif Is Mapped to aa309-316 of $\mathrm{LPA}_{2}-\mathrm{CT}$}

To map out the domain in the $\mathrm{LPA}_{2}$-CT that mediates interaction with the LIM proteins, we first generated deletion mutations that span the $\mathrm{LPA}_{2}-\mathrm{CT}$ region of aa 296351 but kept the PDZ motif intact in the mutants LPA $-\Delta 296, \mathrm{LPA}_{2}-\Delta 302+\mathrm{DSTL}, \mathrm{LPA}_{2}-$ $\Delta 309+$ DSTL, $\mathrm{LPA}_{2}-\Delta 316+\mathrm{DSTL} \mathrm{LPA}_{2}-\Delta 327+\mathrm{DSTL}$, or truncated it in the $\mathrm{LPA}_{2}-$ $\Delta 338+\mathrm{DSTL}, \mathrm{LPA}_{2}-\Delta 347, \mathrm{LPA}_{2}-\Delta 348$ constructs, or mutated the critical leucine residue in the $\mathrm{LPA}_{2}$-L351A construct (Fig. 3.2). DSTL, the last four amino acids of the $\mathrm{LPA}_{2}$, is the PDZ binding motif and required for the interaction of $\mathrm{LPA}_{2}$ with NHERF2. The interaction is reduced by $\mathrm{LPA}_{2}$-L351A point mutant and completely abolished by $\mathrm{LPA}_{2}$ $\Delta \mathrm{STL}$ (Oh, 2004; Li, 2005a). These constructs together with GFP-TRIP6 were reconstituted into HEK 293T cells, which express a barely detectable level of endogenous TRIP6, and exposed to LPA stimulation for $10 \mathrm{~min}$. The co-immunoprecipitation results of these mutants with TRIP6 showed that truncation mutant of $\mathrm{LPA}_{2}-\Delta 309+\mathrm{DSTL}$ and those of more N-terminal ( $\mathrm{LPA}_{2}-\Delta 296$ and $\left.\mathrm{LPA}_{2}-\Delta 302+\mathrm{DSTL}\right)$ could not pull down TRIP6. In contrast, $\mathrm{LPA}_{2}-\Delta 316+\mathrm{DSTL}$ and mutants extending beyong residue 316 retained binding (Fig. 3.3). This indicates that a motif between amino acids 309-316 is required for the interaction of TRIP6 with $\mathrm{LPA}_{2}$.

Previously it has been shown that upon LPA stimulation Siva-1 colocalizes with $\mathrm{LPA}_{2}$ in NIH3T3 fibroblasts (Lin, 2007). To examine association with Siva-1, GFP-Siva1 and $\mathrm{LPA}_{2}$ mutants were cotransfected into SV40 T-antigen transformed $\mathrm{LPA}_{1 / 2}$ double knockout (DKO) MEFs. As shown in Fig. 3.4, Siva-1 (green) was localized to nucleus,

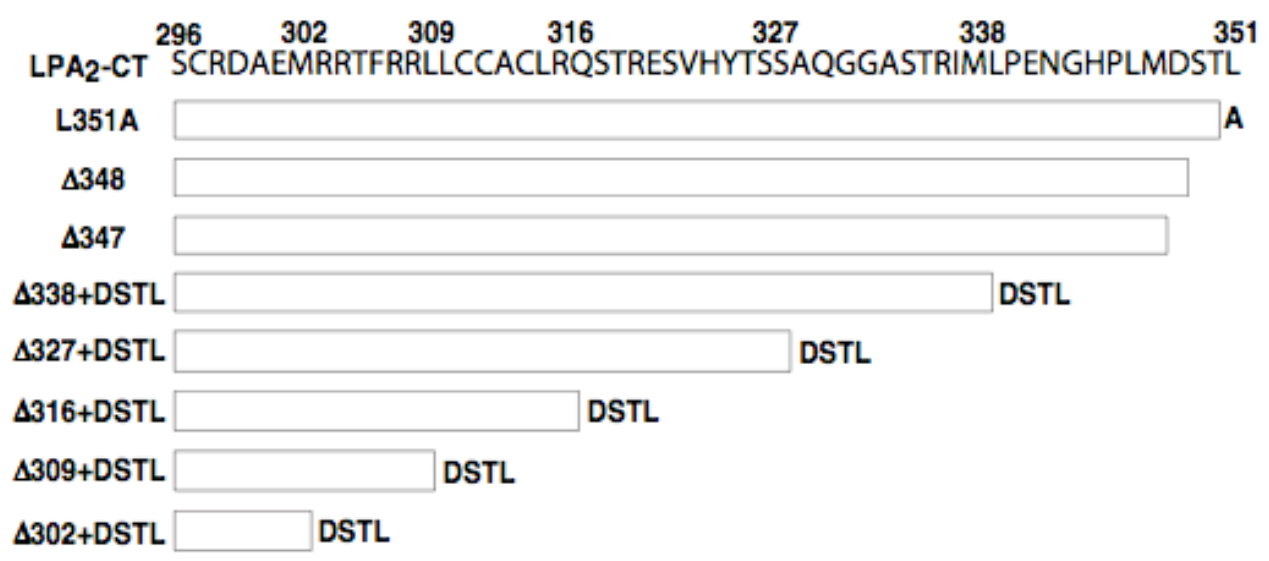

Figure 3.2. Schematic illustration of the $\mathrm{LPA}_{2}-\mathrm{CT}$ deletion constructs. 


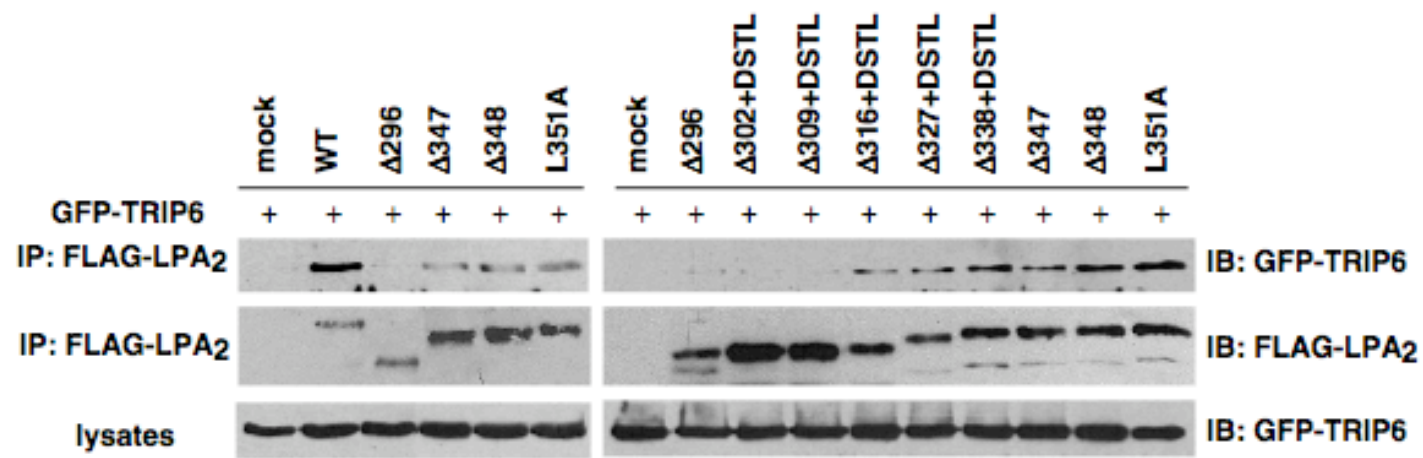

Figure 3.3. TRIP6 binding domain is localized between aa309-316 of $\mathrm{LPA}_{2}-\mathrm{CT}$. GFP-TRIP6 and GST, GST-FLAG-LPA 2 deletion mutant constructs were transfected into HEK293T cells. After starvation in 0.1\% BSA-containing DMEM overnight, cells were incubated without or with $2 \mu \mathrm{M}$ LPA for $10 \mathrm{~min}$. The LPA receptors from the whole lysates were immunoprecipitated (IP) with anti-FLAG M2 monoclonal antibodyconjugated agarose, resolved by SDS-PAGE, and transferred to nitrocellulose membrane for immunoblotting. The immunoblot (IB) was probed with an anti-GFP polyclonal antibody to detect coimmunoprecipitated GFP-TRIP6. The same blot was then stripped and reprobed with an anti-FLAG polyclonal antibody to detect immunoprecipitated $\mathrm{LPA}_{2}$. The bottom panel is an immunoblot showing the expression of GFP-TRIP6 in the whole cell lysates. Also note that mutation or deletion of the PDZ motif did not abolish but weakened the binding of TRIP6 compared to WT LPA 2 . 


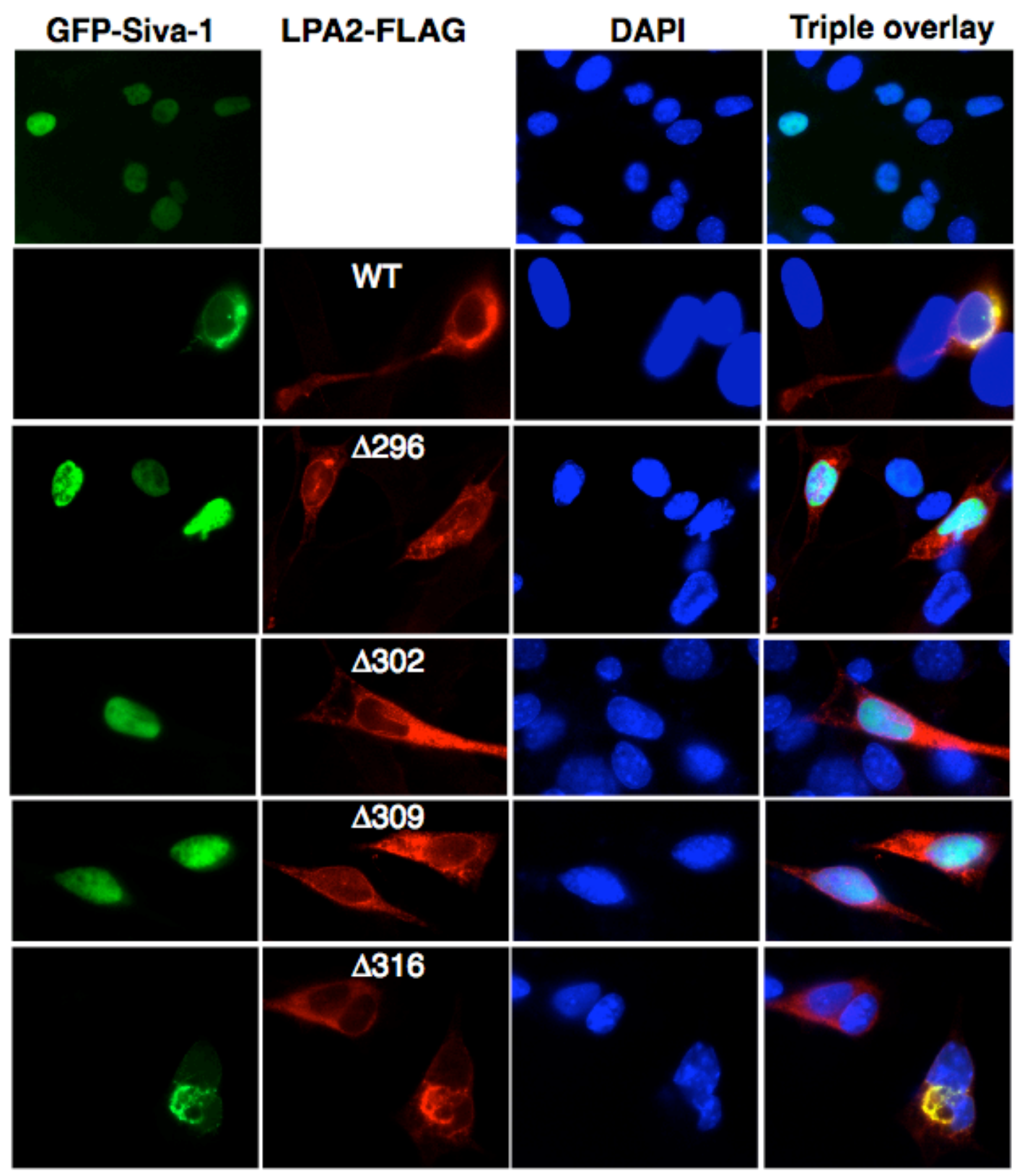

Figure 3.4. Siva-1 binding domain is located between aa309-316 of LPA2-CT. DKO MEFs were transiently transfected with pEGFP-Siva-1 and an expression vector of FLAG-LPA2 or FLAG-LPA2 deletion mutant as indicated. After fixation, permeabilization and blocking, cells were incubated with an anti-FLAG M2 mouse monoclonal antibody followed by the Texas Red-X-conjugated anti-mouse secondary antibody to detect the wild type or mutant of FLAG-LPA2. The nuclei were stained with Hoechst 33258. GFP-Siva-1 was directly visualized by fluorescence microscopy. 
while $\mathrm{LPA}_{2}$ (red) is present at plasma membrane and the cytosol. LPA 2 colocalization with Siva-1 (yellow) was detected in cells expressing WT LPA 2 and $\mathrm{LPA}_{2}$ $\Delta 316+$ DSTL. The same findings were observed in cells expressing $L_{P} A_{2}-\Delta 327+D S T L$ and $\mathrm{LPA}_{2}-\Delta 338+\mathrm{DSTL}$ (data not shown). However, no colocalization was observed in

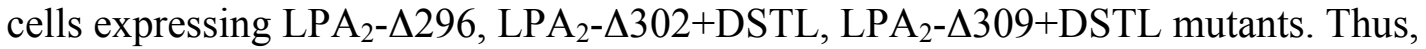
immunocytochemistry revealed the same requirement for Siva-1 binding to $\mathrm{LPA}_{2}$ as we found using coimmunoprecipitation with TRIP6, namely a motif between aa309-316 is required the interaction with Siva-1.

Interestingly, two cysteine residues reside in the region between 309-316, which supports our hypothesis for the role of the zinc-finger like domain in this interaction. To map out the motif in this region, we carried out alanine scanning mutagenesis between residues 296-316.

\subsubsection{The C311xxC314 Motif of the LPA $A_{2}$ Receptor Is Required for Interaction with TRIP6 and Siva-1}

We started with alanine mutation of the conserved amino acids M302, L310, $\mathrm{C} 311$ and $\mathrm{C} 314$ because we hypothesized that some of these residues might play an important role in binding to LIM proteins. These alanine (or also glycine for C314) point mutants together with L351A were cotransfected into HEK 293T cells with myc-TRIP6. As shown in Fig. 3.5A, mutation of Cys311 and/or Cys 314 to alanine, but not other residues as indicated, abolished coimmunoprecipitation of TRIP6. It should be noted that on blots with long exposure, a very weak association of endogenous TRIP6 with C311A or C3114A could still be detected. In the case of Siva-1, C311A and C314A could still pulldown Siva-1, but not the C311AC314A double mutant (Fig. 3.5 B). Also colocalization of Siva-1 with these alanine mutants was examined. C311A and C314A abolished colocalization with Siva-1 (Fig. 3.6). Together, these findings suggest that $\mathrm{C}^{311} \mathrm{xxC}^{314}$ is required for interaction with TRIP6 or Siva-1.

To exclude the requirement of other residues we performed alanine scanning of the domain aa296-316 in addition to those mutants shown above. With these alanine mutants, first we checked cellular (Fig. 3.7) and cell surface expression and ligandinduced $\mathrm{Ca}^{2+}$ activation to make sure that mutation does not cause change of receptor function (Table 3.3). We found two residues D299 and E302, when mutated to alanine, lost their surface expression. All other mutants behaved as WT LPA2. Coimmunoprecipitation with these alanine mutants showed that all of them interacted with TRIP6, though with variation in degree (Fig. 3.8). Taken together, these results demonstrate that the $\mathrm{C}^{311} \mathrm{xxC}^{314}$ motif in the $\mathrm{LPA}_{2}-\mathrm{CT}$ is required for interaction with TRIP6 or Siva-1. 
A

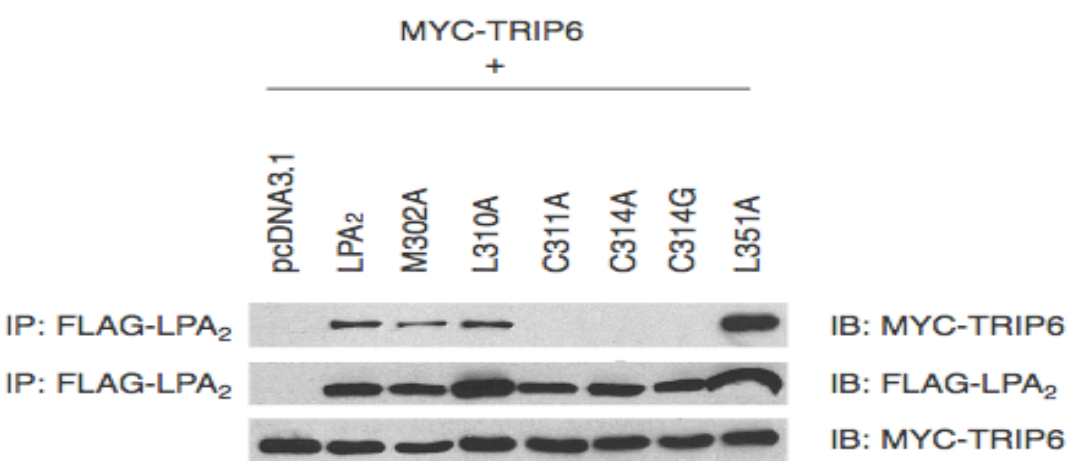

B

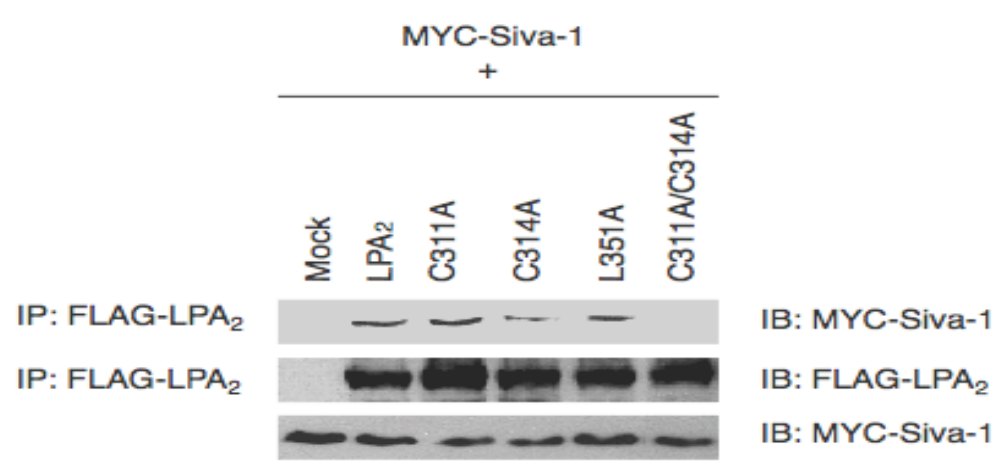

Figure 3.5. The C311-x-x-C314 motif located at the proximal region of the carboxyl terminal tail of the $\mathrm{LPA}_{2}$ receptor regulates the cellular interaction with TRIP6 and Siva-1. (A) HEK 293T cells transiently expressing MYC-TRIP6 with WT $\mathrm{LPA}_{2}$, or its point mutants were starved in $0.1 \%$ BSA containing DMEM for overnight followed by stimulation with $2 \mu \mathrm{M}$ LPA for $10 \mathrm{~min}$. FLAG-LPA 2 , WT or mutant, was immunoprecipitated with the anti-FLAG M2 antibody-conjugated agarose beads, resolved by SDS-PAGE, and the immunoblot was probed with an anti-MYC rabbit polyclonal antibody to detect the coimmunoprecipitated MYC-TRIP6. The blots were stripped and reprobed with an anti-FLAG rabbit polyclonal antibody to detect the FLAG-LPA 2 receptor. The bottom panel in each figure shows the expression of MYCTRIP6 in the total lysates. (B) To detect the cellular interaction of $\mathrm{LPA}_{2}$ with Siva-1, MYC-Siva-1 was coexpressed with WT LPA 2 or its point mutants in HEK 293T cells. After starvation overnight, the cells were harvested and the co-immunoprecipitation was performed as described above. MYC-Siva-1 coimmunoprecipiated with $\mathrm{LPA}_{2}$ was detected by an anti-MYC rabbit polyclonal antibody. The cellular interaction of TRIP6 and $\mathrm{LPA}_{2}$ is LPA-dependent. LPA stimulation for 5-10 min induces the association of TRIP6 with LPA 2 on the membrane. At 15-20 min stimulation with LPA, TRIP6 is translocated to focal adhesions and associated with actin. In contrast, Sival binds to the $\mathrm{LPA}_{2}$ receptor in the absence of LPA. LPA stimulation for 30-60 min down-regulates the expression of both $\mathrm{LPA}_{2}$ and Siva-1. 

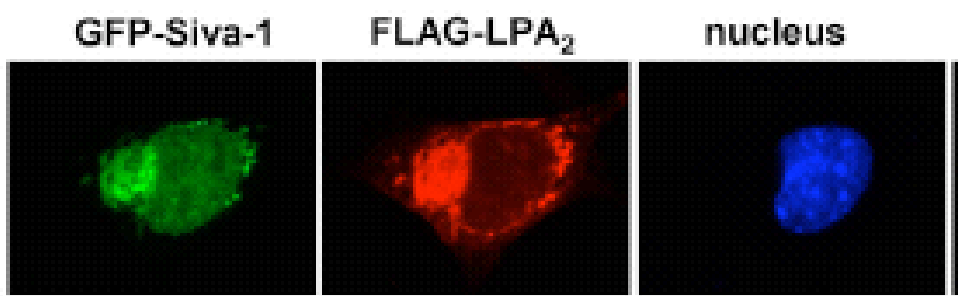

overlay

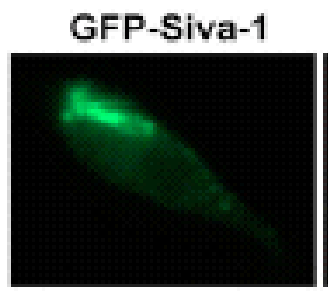

FLAG-C311A

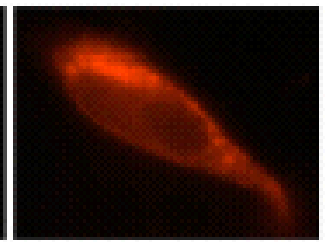

nucleus
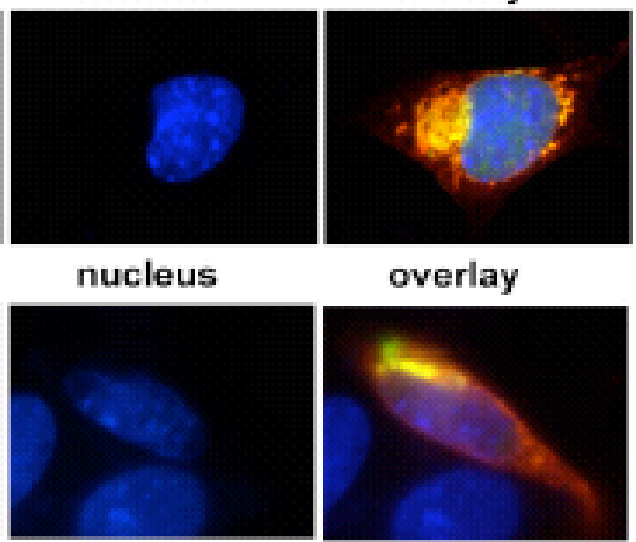

overlay

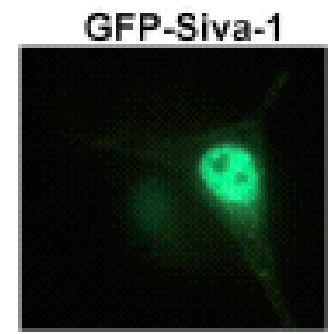

FLAG-C314A

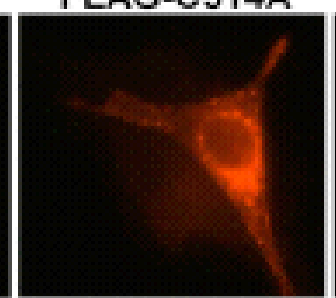

nucleus
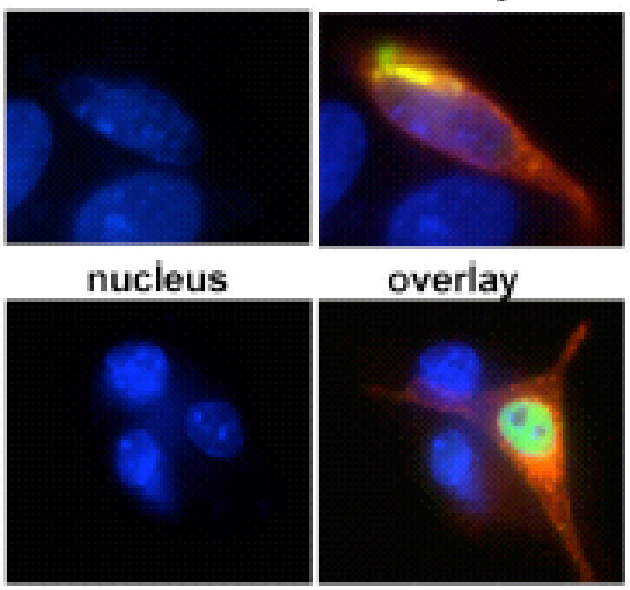

overlay

GFP-Siva-1 FLAG-C311A/C314A

nucleus
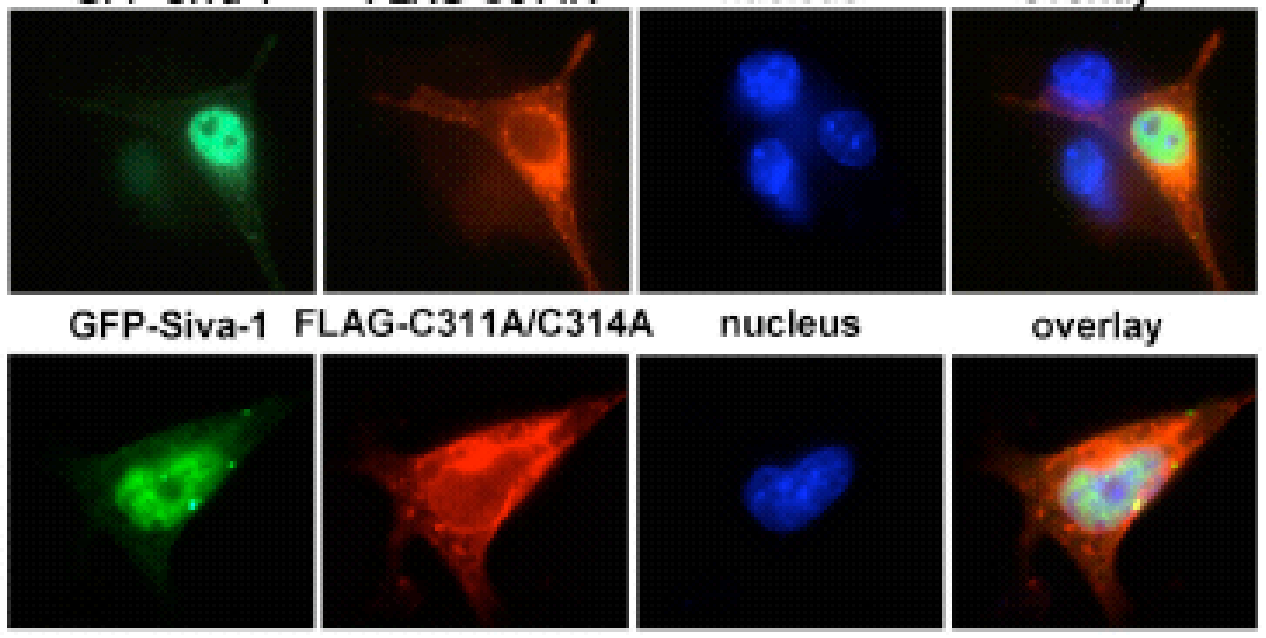

Figure 3.6. Mutation of both Cys311 and Cys314 to Alanine abolishes the colocalization of LPA $\mathbf{A}_{2}$ with Siva-1. The SV40 T-antigen-transformed DKO MEFs were transiently transfected with pEGFP-Siva-1 with an expression vector of FLAG$\mathrm{LPA}_{2}$ or its mutants as indicated. After fixation, permeabilization and blocking, cells were incubated with an anti-FLAG antibody followed by Texas Red-X-conjugated anti-mouse secondary antibody to detect the wild type or mutant of FLAG-LPA 2 GFP-Siva-1 was directly visualized by fluorescence microscopy. 


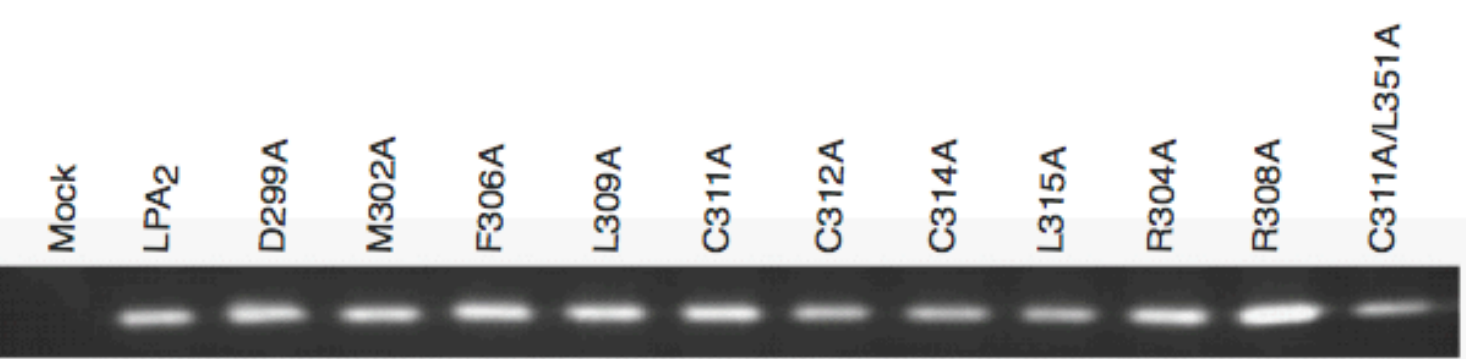
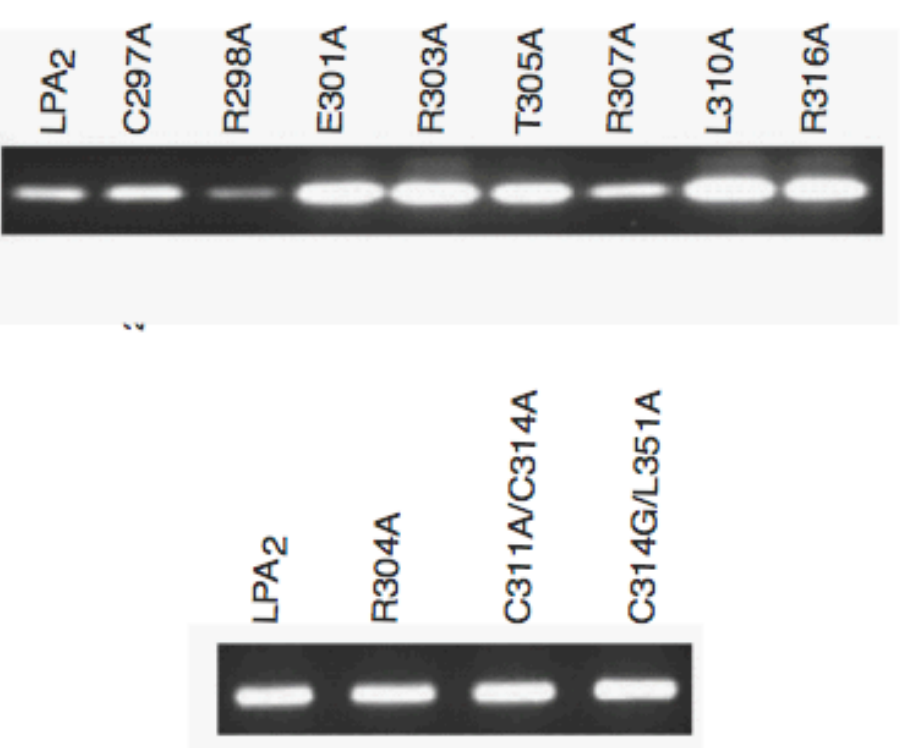

Figure 3.7. Cellular expression of $L P A_{2}-C T$ point mutants in $R H$ cells. $\mathrm{LPA}_{2}$ point mutants as indicated were transfected into RH cells. The LPA receptor from the whole lysates was immunoprecipitated with anti-FLAG M2 monoclonal antibody-conjugated agarose, resolved by SDS-PAGE, and transferred to nitrocellulose membrane for immunoblotting. The immunoblot was probed with an anti-FLAG M2 monoclonal polyclonal antibody to detect immunoprecipitated $\mathrm{LPA}_{2}$. 
Table 3.3. Surface expression and ligand-induced $\mathrm{Ca}^{2+}$ mobilization of $\mathrm{LPA}_{2}-\mathrm{CT}$ mutants transiently expressed in RH cells.

\begin{tabular}{lllcc}
\hline Mutation & Codon & $\begin{array}{l}\text { Mutated } \\
\text { codon }\end{array}$ & $\begin{array}{c}\text { Surface } \\
\text { Exp }^{\#} \\
(\% \text { cells })\end{array}$ & Ca $^{2+}$ peak \\
\hline C297A & TGC & GCT & 25 & $0.63 \pm 0.05$ \\
R298A & CGA & GCA & 17.3 & $0.5 \pm 0.05$ \\
D299A & GAT & GCT & 2.7 & $0.11 \pm 0.04$ \\
E301A & GAG & GCT & 20 & $0.22 \pm 0.06$ \\
M302A & ATG & GCA & 2.5 & $0.13 \pm 0.05$ \\
R303A & CGC & GCT & 22.7 & $0.49 \pm 0.07$ \\
R304A & CGC & GCT & 21.5 & $0.39 \pm 0.06$ \\
T305A & ACC & GCT & 21.7 & $0.53 \pm 0.07$ \\
F306A & TTC & GCT & 13.8 & $0.48 \pm 0.06$ \\
R307A & CGC & GCT & 30.6 & $0.51 \pm 0.09$ \\
R308A & CGC & GCT & 33.2 & $0.35 \pm 0.04$ \\
L309A & CTT & GCT & 13.1 & $0.46 \pm 0.02$ \\
L310A & CTC & GCT & 20.2 & $0.69 \pm 0.03$ \\
C311A & TGC & GCT & 22.7 & $0.67 \pm 0.02$ \\
C312A & TGC & GCT & 32.7 & $0.81 \pm 0.11$ \\
C314A & TGC & GCT & 30.1 & $0.44 \pm 0.05$ \\
L315A & CTC & GCT & 32.2 & $0.46 \pm 0.06$ \\
R316A & CGC & GCT & 24.4 & $0.42 \pm 0.06$ \\
C314AL351A & TGC/CTT & GCT/GCT & 32.9 & $0.43 \pm 0.08$ \\
C314GL351A & TGC/CTT & GGT/GCT & 38.3 & $0.48 \pm 0.08$ \\
C311AC314A & TGC/TGC & GCT/GCT & 35.3 & $0.32 \pm 0.11$ \\
311/314/351A & TGC/TGC/CTT & GCT/GCT/GCT & 26.4 & $0.71 \pm 0.06$ \\
Vector & 3.1/Zeo(+) & & 2.1 & $0.08 \pm 0.05$ \\
WT & hLPA2 & & 40 & $0.69 \pm 0.06$ \\
\hline
\end{tabular}

${ }^{\text {\# }}$ Expression. * In response to $1 \mu \mathrm{M}$ LPA (18:1) 

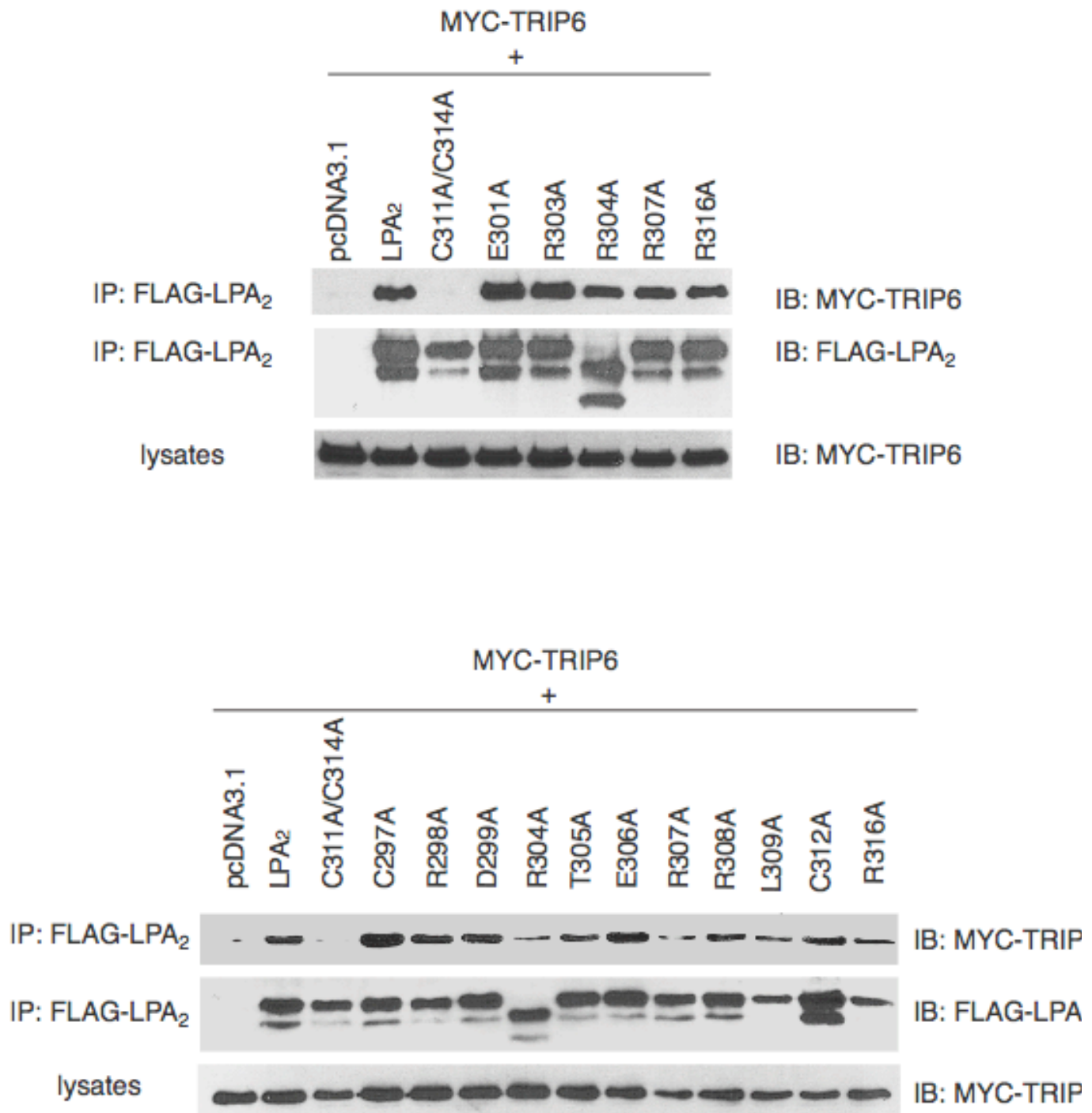

Figure 3.8. Alanine scanning of aa296-316 by coimmunoprecipitation with TRIP6. HEK 293T cells transiently expressing MYC-TRIP6 with WT LPA 2 , or its point mutants as indicated were starved in $0.1 \%$ BSA containing DMEM for $8 \mathrm{~h}$ followed by stimulation with $2 \mu \mathrm{M}$ LPA for $10 \mathrm{~min}$. The FLAG-tagged receptor constuct was immunoprecipitated with the anti-FLAG M2 antibody-conjugated agarose beads, resolved by SDS-PAGE, and blot was probed with an anti-MYC rabbit polyclonal antibody to detect the coimmunoprecipitated MYC-TRIP6. The blots were stripped and reprobed with an anti-FLAG rabbit polyclonal antibody to detect the FLAG-LPA 2 receptor. The bottom panel in each figure shows the expression of MYC-TRIP6 in the lysates. 


\subsubsection{LPA $\mathrm{LA}_{2}$ Receptor Directly Interacts with TRIP6 and Siva-1 via the C311xxC314 Motif}

We next performed pull down experiments using purified recombinant proteins to examine the role of the $\mathrm{C}^{311} \mathrm{xxC}^{314}$ motif in the direct interaction with TRIP6 and Siva-1. The purified TRIP6 was pulled down by GST-LPA 2 -CT. However, none of C311A, C314A, C311A/C314A, C314A/L351A mutants and GST protein alone formed a complex (Fig. 3.9a). This indicates that the $\mathrm{C}^{311} \mathrm{xxC}^{314}$ motif is required for the direct interaction with TRIP6. In the case of Siva-1 (Fig. 3.9b), C311A or C314A mutant could still pull down some Siva-1, whereas C311A/C314A double mutant did not form a complex. This indicates Siva-1 binds more tightly to LPA 2 than TRIP6 so that neither C311A nor C314A alone abolished the association. These finding indicate that the $\mathrm{LPA}_{2}-$ $\mathrm{CT}$ interacts directly with TRIP6 and Siva-1 via $\mathrm{C}^{311} \mathrm{xxC}^{314}$ motif.

\subsubsection{Palmitoylation Does Not Affect the Interaction of LPA $A_{2}$ with TRIP6/Siva-1}

GPCR are known to be subject to post-translational lipid modifications mainly through palmitoylation that includes the attachment of palmitate to one or more of the cysteine residues through a thioester bonds. Palmitoylation of GPCR plays important roles in receptor binding, targeting, trafficking, signaling and protein interactions (Dunphy \& Linder 1998; Qanbar \& Bouvier, 2003 review; Escriba, 2007). Palmitoylation typically occurs on the cysteine residues proximal to the cytosolic end of the seventh transmembrane helix. The identification of the role of the $\mathrm{C}^{311} \mathrm{xxC}^{314}$ motif in $\mathrm{LPA}_{2}-\mathrm{CT}$ prompted us to investigate whether palmitoylation occurs on these cysteines and if yes, what role it plays in the interaction between LIM domain proteins and LPA 2 . WT LPA 2 and its Cys to Ala mutants, including residues Cys297, Cys311, Cys312 and Cys314 were transfected into HEK 293T cells. Cells were labled with $\left[{ }^{3} \mathrm{H}\right]$-palmitic acid at $37{ }^{\circ} \mathrm{C}$ for 2 $\mathrm{h}$. The receptor was immunoprecipitated using anti-FLAG agarose as above, separated by SDS-PAGE and the immunoprecipitated protein was visualized by western blotting, whereas the incorporated $\left[{ }^{3} \mathrm{H}\right]$-palmitate was detected by autoradiography. The result showed that $\mathrm{C} 311 \mathrm{~A} / \mathrm{C} 314 \mathrm{~A}$ and $\mathrm{C} 311 \mathrm{~A} / \mathrm{C} 312 \mathrm{~A} / \mathrm{C} 314 \mathrm{~A}$ mutants have about $80 \%$ reduction of palmitoylation compared to WT (Fig 3.10A, B). However, even in the $\mathrm{C} 311 \mathrm{~A} / \mathrm{C} 312 \mathrm{~A} / \mathrm{C} 314 \mathrm{~A}$ mutant there is still detectable level of palmitoylation, indicating palmitoylation in cysteines outside of the $\mathrm{LPA}_{2}-\mathrm{CT}$. Taken together, C311 and C314 constitute sites of $\mathrm{LPA}_{2}-\mathrm{CT}$ palmitoylation.

To examine the effect of palmitoylation in the interaction of $\mathrm{LPA}_{2}$ with TRIP6 and Siva-1, 2-bromopalmitate (2-BP), a non-metabolizable palmitate analog that blocks palmitate incorporation into proteins, was used to block LPA 2 palmitoylation (Webb, 2000). As shown in Fig 3.11, $100 \mu \mathrm{M}$ 2-BP treated for 30 min decreased the $\mathrm{LPA}_{2}$ palmitoylation level to $20 \%$ of the control.

Next we examined the effect of palmitoylation on the interaction of $\mathrm{LPA}_{2}$ with TRIP6 and Siva-1 by coimmunoprecipitation after treating the cells with $100 \mu \mathrm{M} 2$-BP for $4 \mathrm{~h}$. the precipitated $\mathrm{LPA}_{2}$ receptor was pre-treated with N-ethylmaleimide (NEM) 
A
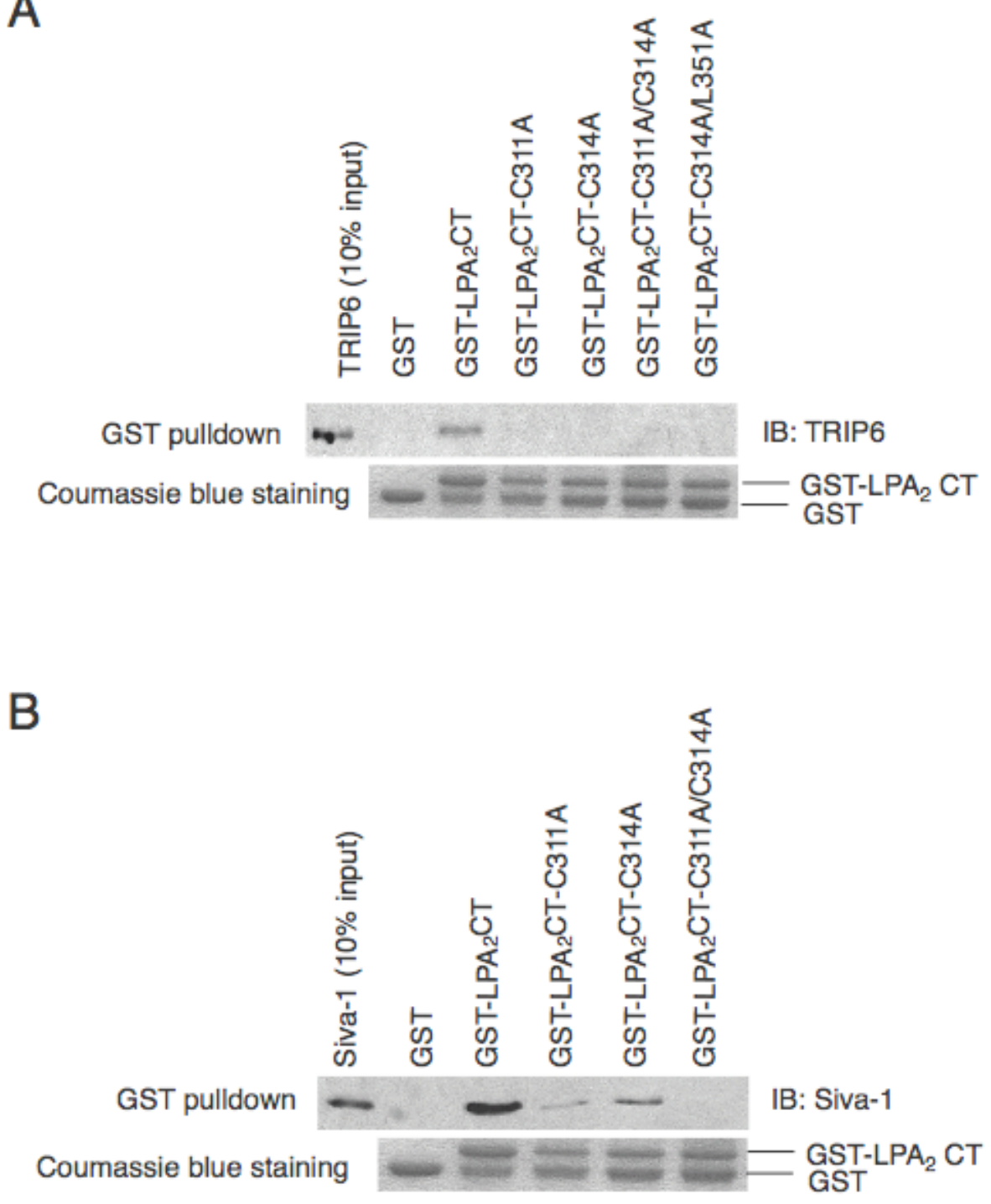

Figure 3.9. Mutation of $\mathrm{LPA}_{2}$-CT Cys311 or Cys314 to Alanine abolishes its direct binding to TRIP6; however, only when both are mutated binding to Siva-1 is completely eliminated. GST-TRIP6 or GST-Siva-1 was purified from E. coli transformed with pGEX-6P3-TRIP6 or pGEX-6P3-Siva-1, and further digested with Precision Protease to cleave GST. One $\mu \mathrm{g}$ of TRIP6 or $0.1 \mu \mathrm{g}$ of Siva-1 was incubated with one $\mu \mathrm{g}$ of GST or GST fusion protein of LPA ${ }_{2}$ CT (aa 296-351), LPA ${ }_{2}$ CT-C311A, $\mathrm{LPA}_{2} \mathrm{CT}-\mathrm{C} 314 \mathrm{~A}$ or LPA $2 \mathrm{CT}-\mathrm{C} 311 \mathrm{~A} / \mathrm{C} 314 \mathrm{~A}$ at $4{ }^{\circ} \mathrm{C}$ for $3 \mathrm{~h}$. TRIP6 (A) or Siva-1 (B) pulled down by GST fusion proteins was resolved by SDS-PAGE and detected with an antibody specific to TRIP6 or Siva-1, respectively. The first lane shows the $10 \%$ input of TRIP6 or Siva-1 used in these experiments. 
A
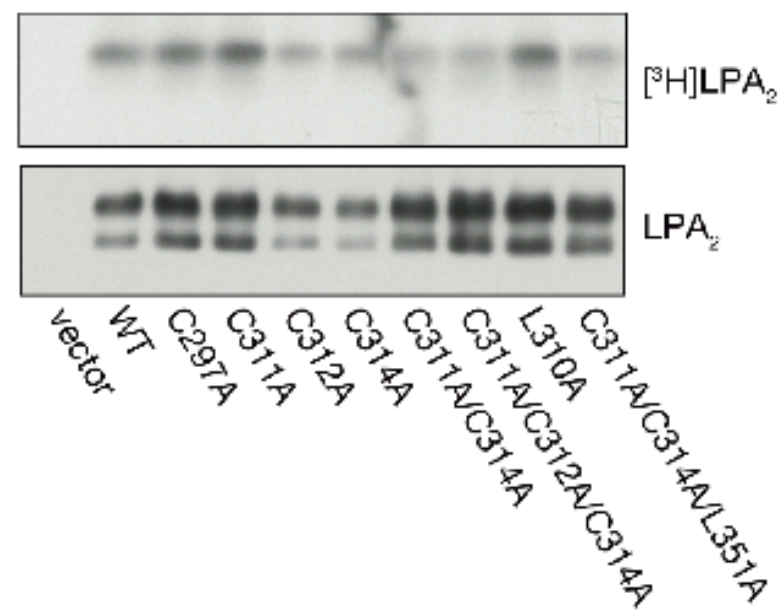

B

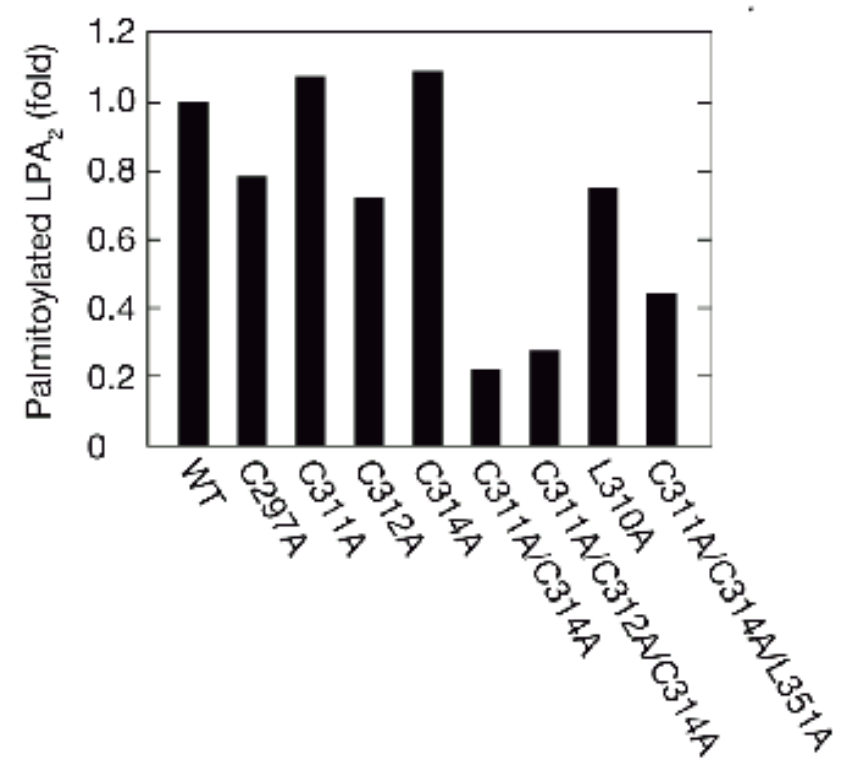

Figure 3.10. Mutation of $\mathrm{LPA}_{2}-\mathrm{CT}$ cysteine residues inhibits receptor

palmitoylation. (A) HEK $293 \mathrm{~T}$ cells were transfected with the plasmids as indicated. Cells were labeled with $\left[{ }^{3} \mathrm{H}\right]$ palmitic acid at $37{ }^{\circ} \mathrm{C}$ for $2 \mathrm{~h}$. The FLAG-LPA 2 receptor was immunoprecipitated with an anti-FLAG M2 agarose beads, resolved by SDS-PAGE and detected by autoradiography (top panel) and an anti-FLAG antibody (bottom panel), respectively. (B) The relative levels of palmitoylation of each mutant compared to the wild-type $\mathrm{LPA}_{2}$ receptor after normalization by total receptor expression. 


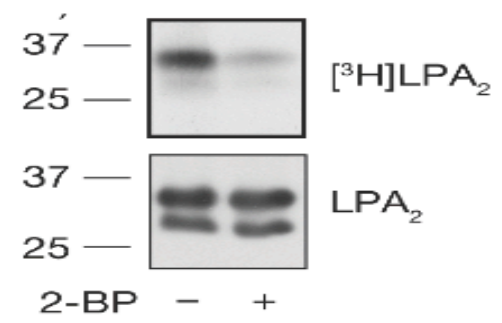

Figure 3.11. 2-bromopalmitate inhibits LPA 2 palmitoylation. HEK 293T cells expressing FLAG-LPA 2 was pretreated with $100 \mu \mathrm{M}$ 2-BP for 30 min followed by the labeling with $\left[{ }^{3} \mathrm{H}\right]$ palmitic acid at $37^{\circ} \mathrm{C}$ for $2 \mathrm{~h}$. The FLAG-LPA 2 receptor was immunoprecipitated with an anti-FLAG agarose beads, resolved by SDS-PAGE and detected by autoradiography (top panel) and an anti-FLAG antibody (bottom panel), respectively. 
followed by the acyl-biotinyl exchange with hydroxylamine and biotin-HPDP. The biotinylated FLAG-LPA 2 receptor was eluted from the beads using excess FLAG peptides for competition, and then precipitated with avidin beads. After SDS-PAGE, the immunoblot was probed with an anti-FLAG antibody to detect the palmitoylated FLAG$\mathrm{LPA}_{2}$ receptor. 2-BP caused no change in the amount of either Siva-1 or TRIP6 pulldown by FLAG-LPA ${ }_{2}$ to vehicle, even though the palmitoylation of $\mathrm{LPA}_{2}$ was completely blocked by 2-BP at $4 \mathrm{~h}$ (Fig. 3.12). This suggests that palmitoylation in $\mathrm{LPA}_{2}$ does not interfere with the association of $\mathrm{LPA}_{2}$ with TRIP6 or Siva-1. These results expand our in vitro studies with the non-palmitoylated $E$. Coli-derived recombinant proteins that showed strong interactions between $\mathrm{LPA}_{2}$ and the LIM proteins.

\subsubsection{Characterization of Lentivirus-transduced Stable MEFs Expressing hLPA WT and Mutants Defective in Interaction with PDZ or LIM Domain Proteins}

To examine the interaction in a physiological context and study the role of interaction of LPA 2 with TRIP6 and Siva- 1 and PDZ proteins in $\mathrm{LPA}_{2}$-mediated antiapoptosis, we generated stable cell lines expressing WT LPA 2 and mutants in spontaneously immortalized DKO MEFs by lentiviral transduction (Fig. 3.13). The DKO MEFs provide a cell platform for the study of $\mathrm{LPA}_{2}$ function via reconstitution because these DKO MEFs only express $\mathrm{LPA}_{4}$ and $\mathrm{LPA}_{7}(\mathrm{p} 2 \mathrm{y} 5)$ detected by real-time PCR but not the other EDG-family receptors (Fig. 3.14). Furthermore, DKO MEFs show no LPAinduced $\mathrm{Ca}^{2+}$ mobilization, ERK1/2 or Akt phosphorylation in response to LPA stimulation (Contos, 2002 and our findings). In addition, the advantage of lentiviral transduction with the ability to control levels of expression near physiological level of the transduced gene by titration of the virus allows valid comparison between different lines expressing different constructs. The lentiviral constructs for mutants we generated included C311A/C314A, L351A and C311A/C314A/L351A. RT-PCR showed similar expression levels between different stable MEFs and the level is comparable to endogenous $\mathrm{LPA}_{2}$ expressed in WT MEFs. The parental as well as GFP-transduced DKO MEFS do not express $\mathrm{LPA}_{2}$ and served as negative controls (Fig.3.15A). Expression of receptor RNA constructs was confirmed by RT-PCR (Fig 3.15A) and quantitative real time PCR, which showed the similar expression of the receptors in mRNA (Fig 3.15B). Comparable receptor protein expression was demonstrated by immunoprecipitationimmunoblotting (Fig 3.16A). Flow cytometry was carried out to examine cell surface expression of the receptor mutants. As shown in Fig 3.16B, the level of cell surface expression between these stable lines was highly comparable, indicating that these mutations did not affect receptor targeting to the plasma membrane in the DKO MEFs.

$\mathrm{LPA}_{2}$ couples to $\mathrm{G}_{\mathrm{q}}$ and $\mathrm{G}_{\mathrm{i}}$ heterotrimeric $\mathrm{G}$ proteins, which both are involved in mediating LPA-induced $\mathrm{Ca}^{2+}$ mobilization. Ligand-induced $\mathrm{Ca}^{2+}$ mobilization assays showed that the maximal response to saturating LPA concentrations $\left(\mathrm{E}_{\max }\right)$ and the halfmaximally effective concentration ( $\left(\mathrm{EC}_{50}\right)$ were not different among these stable clonal lines and the WT LPA 2 (Fig. 3.17). This indicates that the C-terminal mutations does not affect receptor coupling to $\mathrm{G}_{\mathrm{q} \& \mathrm{i}}$ proteins when expressed in DKO MEFs. There was no detectable $\mathrm{Ca}^{2+}$ response in the non-transduced or mock-transduced DKO MEFs, which was consistent with a previous publication (Contos, 2002). 


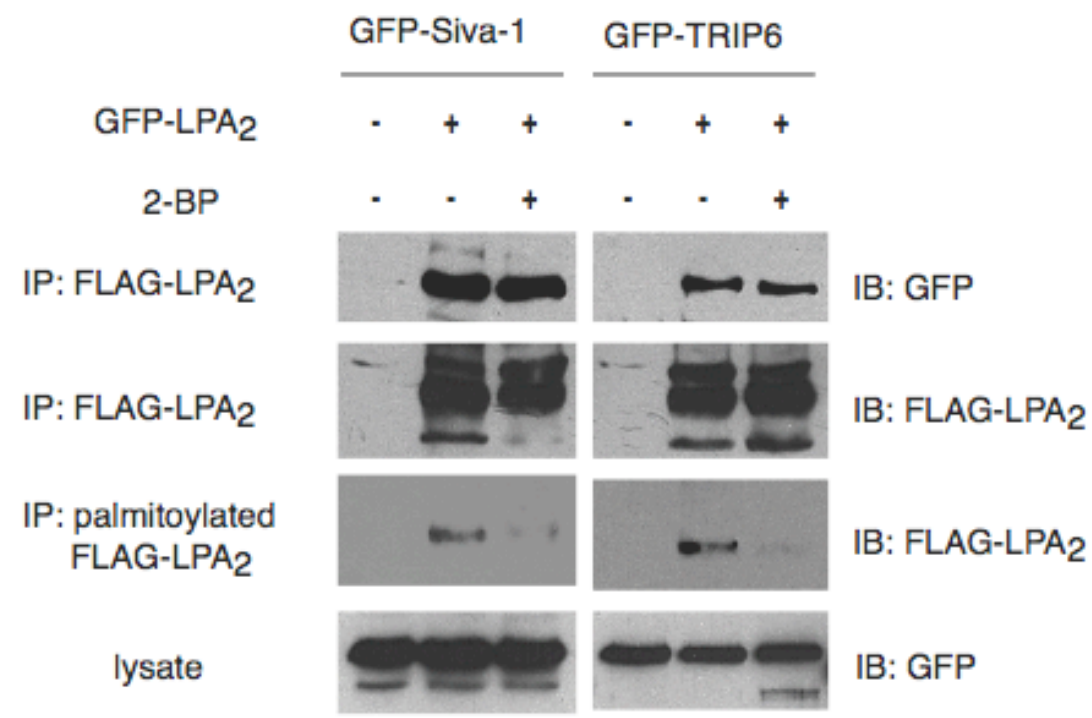

Figure 3.12. Palmitoylation is not required for interaction of $L P A_{2}-C T$ with TRIP6 or Siva-1. GFP-Siva-1 or GFP-TRIP6 was co-expressed with the FLAG-LPA 2 receptor in HEK $293 \mathrm{~T}$ cells. After treatment with $100 \mu \mathrm{M}$ 2-BP in 0.1\% BSA-containing DMEM for $4 \mathrm{~h}$, cells were stimulated with LPA for $10 \mathrm{~min}$, and the FLAG-LPA 2 receptor was immunoprecipitated with the anti-FLAG antibody-conjugated agarose beads. Half of the precipitates were resolved by SDS-PAGE and the immunoblot was probed with an antiGFP polyclonal antibody to detect GFP-Siva-1 and GFP-TRIP6, respectively. The rest of the samples were subjected to acyl-biotinyl exchange procedure to determine the levels of palmitoylated $\mathrm{LPA}_{2}$ receptor. Briefly, the precipitated $\mathrm{LPA}_{2}$ receptor was pre-treated with NEM followed by the acyl-biotinyl exchange with hydroxylamine and biotin-HPDP. The biotinylated FLAG-LPA 2 receptor was eluted from the beads using excess FLAG peptides for competition, and then precipitated with avidin beads. After SDS-PAGE, the immunoblot was probed with an anti-FLAG antibody to detect the palmitoylated FLAG$\mathrm{LPA}_{2}$ receptor. 


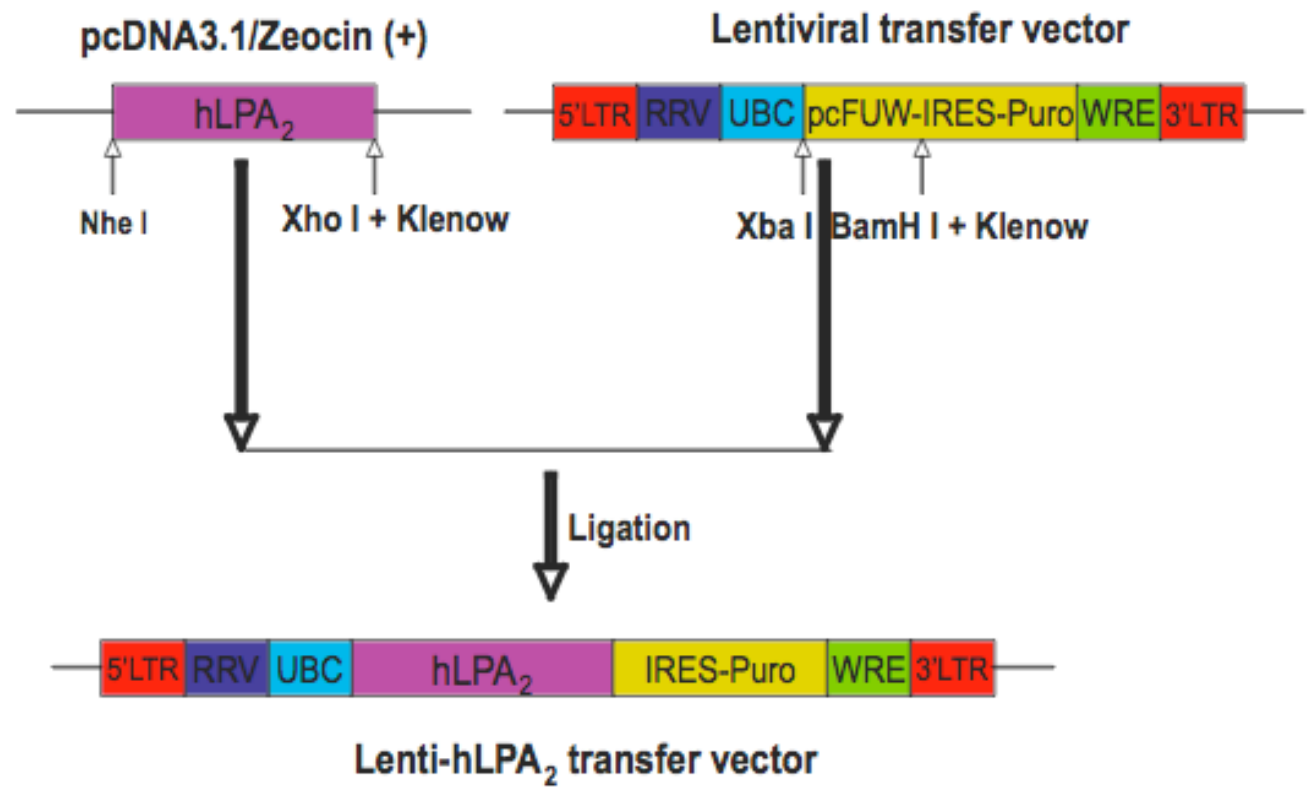

Figure 3.13. Schematic illustration of lentiviral $\mathrm{LPA}_{2}$ constructs.

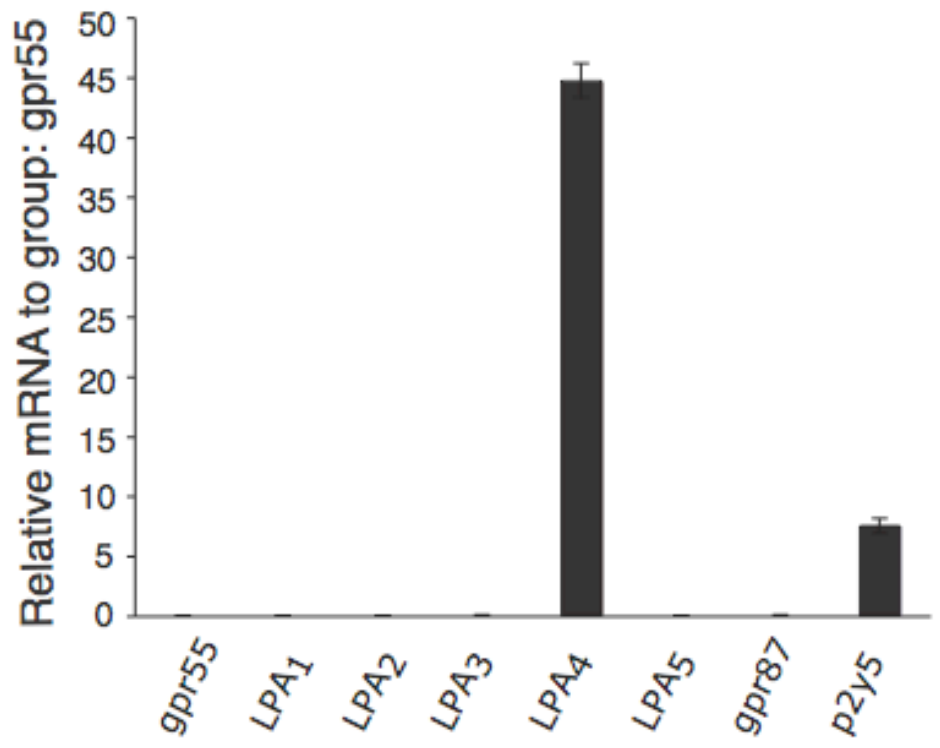

Figure 3.14. mRNA expression profile of LPA receptors in spontaneously immortalized LPA $1 / 2$ DKO MEFs. 
A

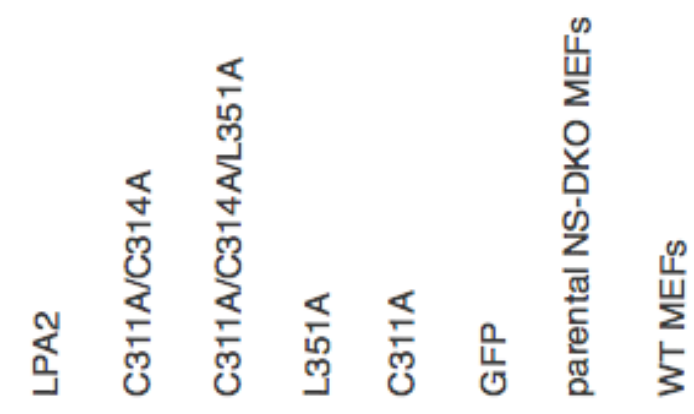

$\longrightarrow=2 \longrightarrow \mathrm{LPA}_{2}$

W

B

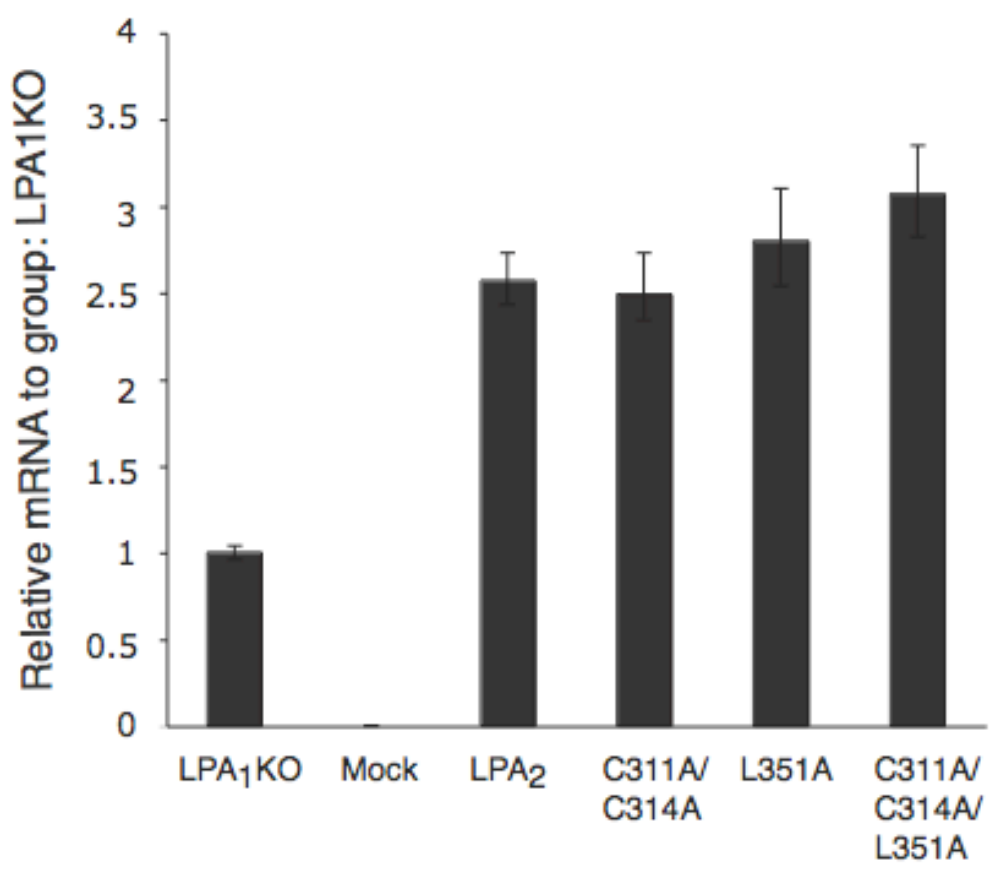

Figure 3.15. mRNA expression of $W T L P A_{2}$ and its mutants in lentivirustransduced DKO MEFs. Lentivirus carrying the FLAG-LPA 2 receptor constructs with mutants C311A/C314A, L351A or C311A/C314A/L351A were stably transduced into DKO MEFs. The expression of $\mathrm{LPA}_{2}$ receptor mRNA was determined by semiquantitative RT-PCR analysis (A) and real-time PCR (B). 
A

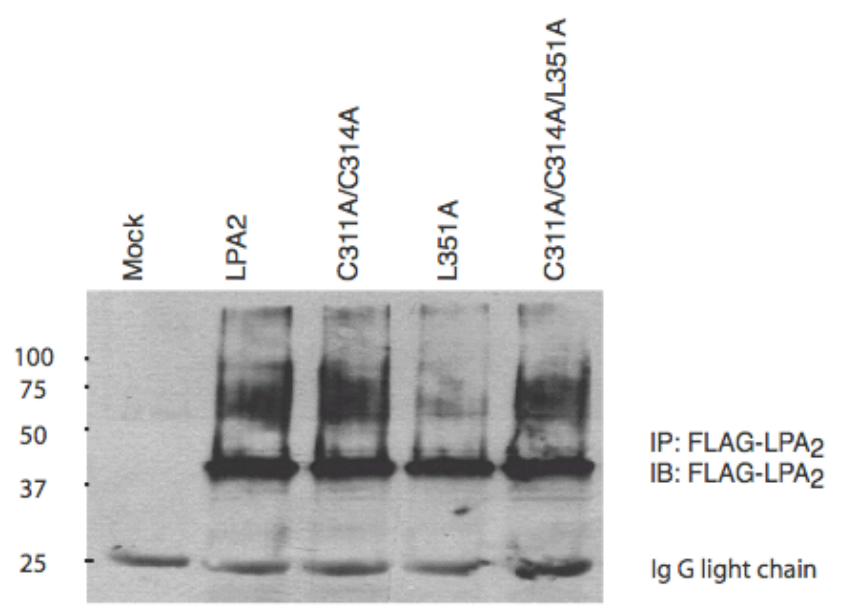

B

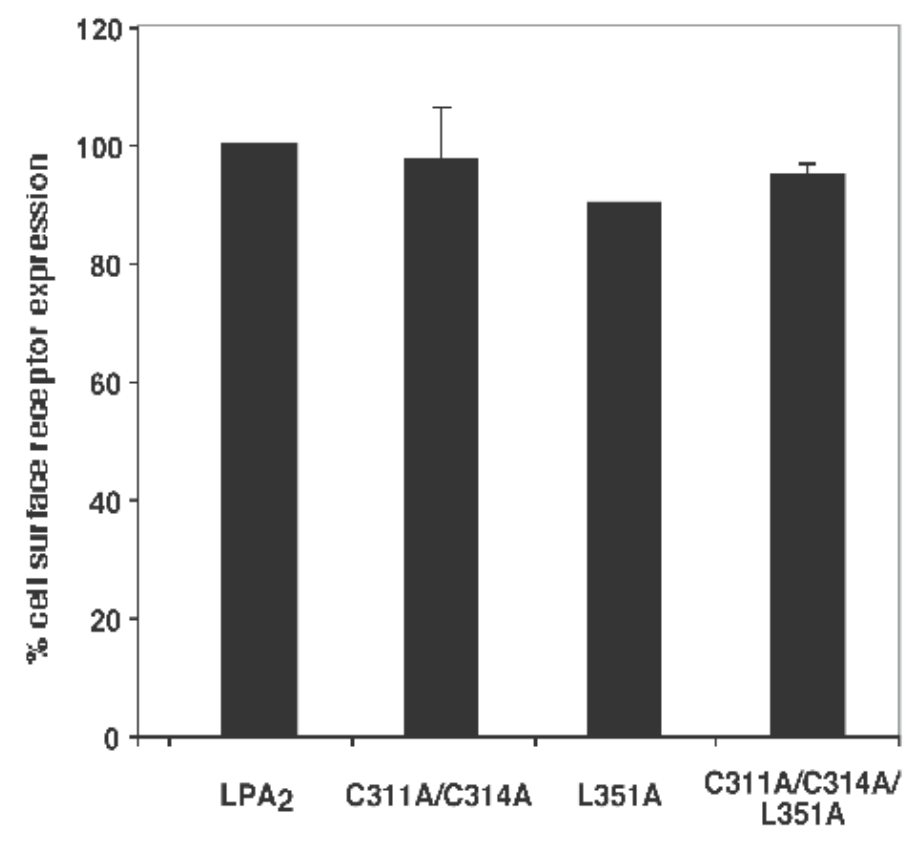

Figure 3.16. Cellular protein and cell surface expression of WT LPA 2 and its mutants in lentivirus-transduced DKO MEFs. Lentiviruses carrying the FLAG-LPA 2 receptor constructs with mutants $\mathrm{C} 311 \mathrm{~A} / \mathrm{C} 314 \mathrm{~A}, \mathrm{~L} 351 \mathrm{~A}$ or $\mathrm{C} 311 \mathrm{~A} / \mathrm{C} 314 \mathrm{~A} / \mathrm{L} 351 \mathrm{~A}$ were stably transduced into DKO MEFs. (A) The $\mathrm{LPA}_{2}$ receptor protein in the whole cell lysates $(1.2 \mathrm{mg})$ was determined by immunoprecipitation with the anti-FLAG M2 antibody-conjugated agarose beads followed by immunoblotting with an anti-FLAG polyclonal antibody. (B) Cells were incubated on ice with an anti-FLAG M2 monoclonal antibody for $1 \mathrm{~h}$ followed by the FITC-conjugated anti-mouse secondary antibody for another hour. After fixation, flow cytometry was performed to determine receptor expression. Data shown is the mean \pm S.E. compared to the $\mathrm{WT}^{\mathrm{LPA}} \mathrm{A}_{2}$ levels in three independent experiments. 


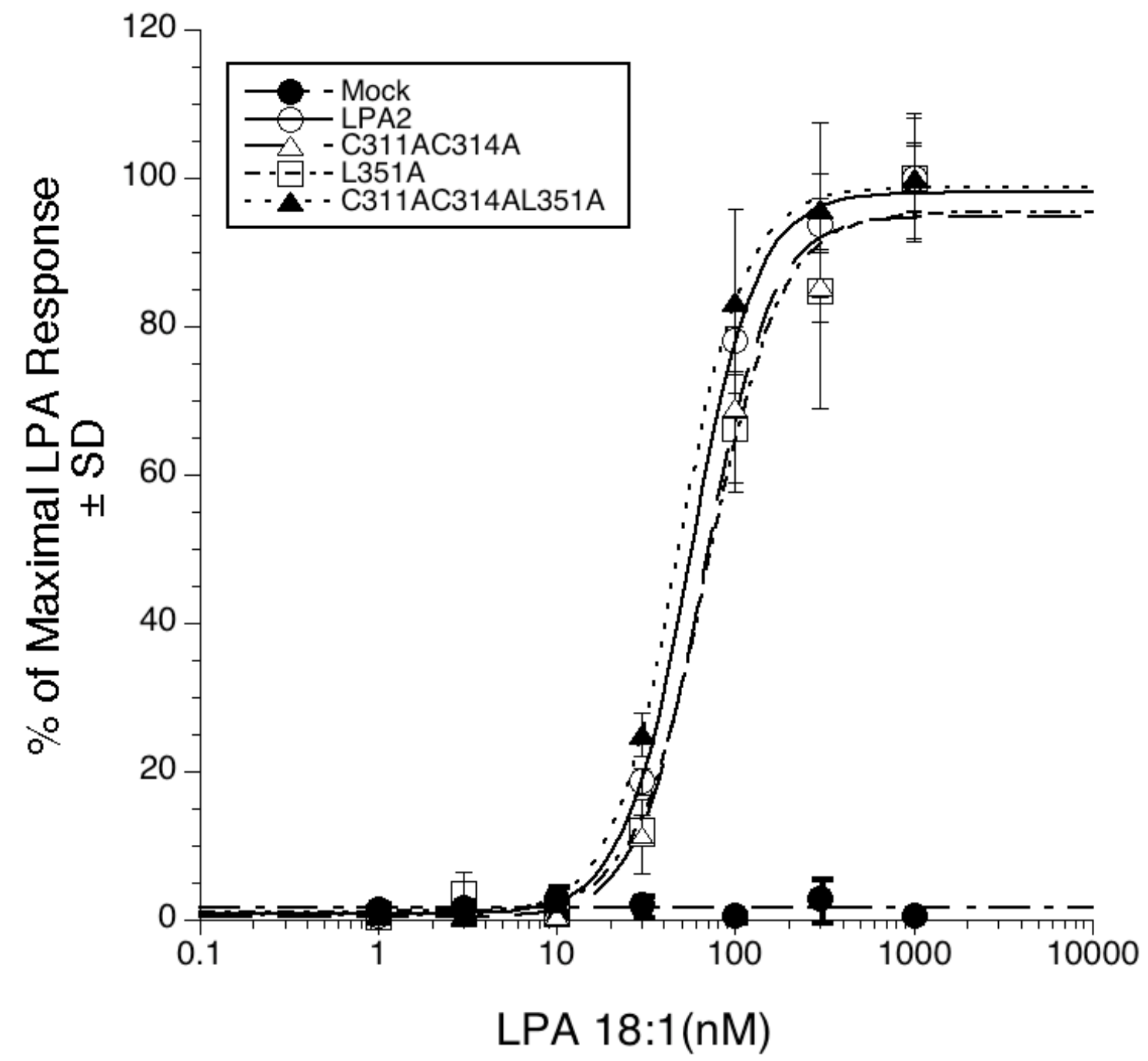

Figure 3.17. Mutation of CxxC and/or PDZ motif(s) of LPA $\mathrm{A}_{2}-\mathrm{CT}$ does not affect ligand-induced $\mathrm{Ca}^{2+}$ mobilization. The spontaneously immortalized DKO MEFs stably expressing mock, WT LPA $, \mathrm{LPA}_{2}-\mathrm{C} 311 \mathrm{~A} / \mathrm{C} 314 \mathrm{~A}, \mathrm{LPA}_{2}-\mathrm{L}_{3} 1 \mathrm{~A}$ or $\mathrm{LPA}_{2}-$ $\mathrm{C} 311 \mathrm{~A} / \mathrm{C} 314 \mathrm{~A} / \mathrm{L} 351 \mathrm{~A}$ by lentiviral transduction were loaded with Fura-2/AM at $37^{\circ} \mathrm{C}$ for $1 \mathrm{~h}$ and monitored in a FLEXstation at excitation wavelengths of 340/380 nm and emission wavelength of $510 \mathrm{~nm}$ for $70 \mathrm{sec}$ after the addition of LPA 18:1 at concentrations indicated. The curves for WT $\mathrm{LPA}_{2}$ and mutants were generated by normalizing the $\mathrm{Ca}^{2+}$ peak at various dilutions to the response elicited by the highest concentration of LPA $(1 \mu \mathrm{M})$ applied. The values of the mock-transduced MEFs were normalized to that of WT $\mathrm{LPA}_{2}$-transduced MEFs. 
Previously others and we have shown that ERK and Akt activation is required for the anti-apoptotic protection of $\mathrm{LPA}_{2}$ (E, 2007; Rusovici, 2007). We hypothesized that mutation of the LIM or PDZ interaction motif of $\mathrm{LPA}_{2}$ might affect antiapoptotic signals mediated thorugh these kinase pathways. We tested ERK1/2 and Akt activation in the lentivirus-transduced stable MEFs that express WT LPA $\mathrm{W}_{2}$ and its mutants. As shown in Fig. 3.18, in mock transduced MEFs there was no detectable ERK1/2 phosphorylation by $1 \mu \mathrm{M}$ LPA treatment for $10 \mathrm{~min}$. ERK $1 / 2$ activation was greatly reduced in C311A/C314A, and L351A mutant-expressing cells and almost completely abolished in the C311A/C314A/L351A mutant compared with WT LPA 2 . Similarly, the activation of Akt by $1 \mu \mathrm{M}$ LPA treatment for 10 min showed the same pattern as LPA-induced ERK1/2 activation. These data indicate that disruption of $\mathrm{LPA}_{2}$ interaction with LIM or PDZ binding domain proteins attenuates ERK and Akt signaling, which are important for antiapoptotic signaling.

\subsubsection{LPA $\quad$-mediated Protection from DNA Damage-induced Apoptosis Is Regulated by the CxxC and PDZ-binding Motifs}

The finding of impaired ERK1/2 and Akt activation (Fig. 3.18) together with the role of $\mathrm{LPA}_{2}$ in mediating proapoptotic function of Siva-1 led us to hypothesize that disruption of $\mathrm{LPA}_{2}$ interaction with either the LIM binding or PDZ binding proteins will attenuate its antiapoptotic effect. To test the apoptotic properties of the LPA $\mathrm{A}_{2}$ mutants expressed in DKO MEFs we performed Annexin V staining following the induction of apoptosis using $1.5 \mu \mathrm{M}$ adriamycin for $14 \mathrm{~h}$. Consistent with the alteration in ERK1/2 and Akt signaling in the C311AC314A stable MEFs, apoptotic protection was impaired in MEFs expressing this mutant (Fig. 3.19). However, LPA still elicited significant protection against adriamycin-induced apoptosis in L351A MEFs, albeit to a reduced extent compared to WT LPA 2 . There was no protection in the MEF expressing the C311AC314AL351A mutant. These findings support the hypothesis for the role of these $\mathrm{C}$ terminal protein-protein interactions in antiapoptotic protection.

To substantiate the finding with annexin V expression, we carried out DNA fragmentation assay in these MEF cell lines. To activate LPA $_{2}$ we applied $1 \mu \mathrm{M} \mathrm{LPA} 1 \mathrm{~h}$ after adriamycin-induced activation of the intrinsic apoptotic pathway. LPA protected 2 $\mu \mathrm{M}$ adriamycin-induced DNA fragmentation in stable lines expressing WT LPA 2 , and C311AC314A while not in mock, L351A or C311AC314AL351A-expressing lines (Fig. 3.20). Thus DNA fragmentation assays confirmed that disruption of these protein-protein interactions abolishes the anti-apoptotic effect of $\mathrm{LPA}_{2}$. Of note, is that in the mocktransduced MEFs, DNA fragmentation was high in the serum starvation group, indicating the protective effect of $\mathrm{LPA}_{2}$ against serum starvation-induced apoptosis.

Several lines of evidence demonstrate that Siva-1-induced apoptosis is mediated by the mitochondrial pathway (Prasad 1997; Py, 2004; Chu 2004, 2005). Moreover, overexpression of Siva-1 promotes adriamycin-induced procaspase 3 cleavage, and this effect is reduced by LPA pretreatment (Lin, 2007). We hypothesized that Siva-1 levels in the cytoplasm and adriamycin-induced procaspase- 3 cleavage will be affected in those 

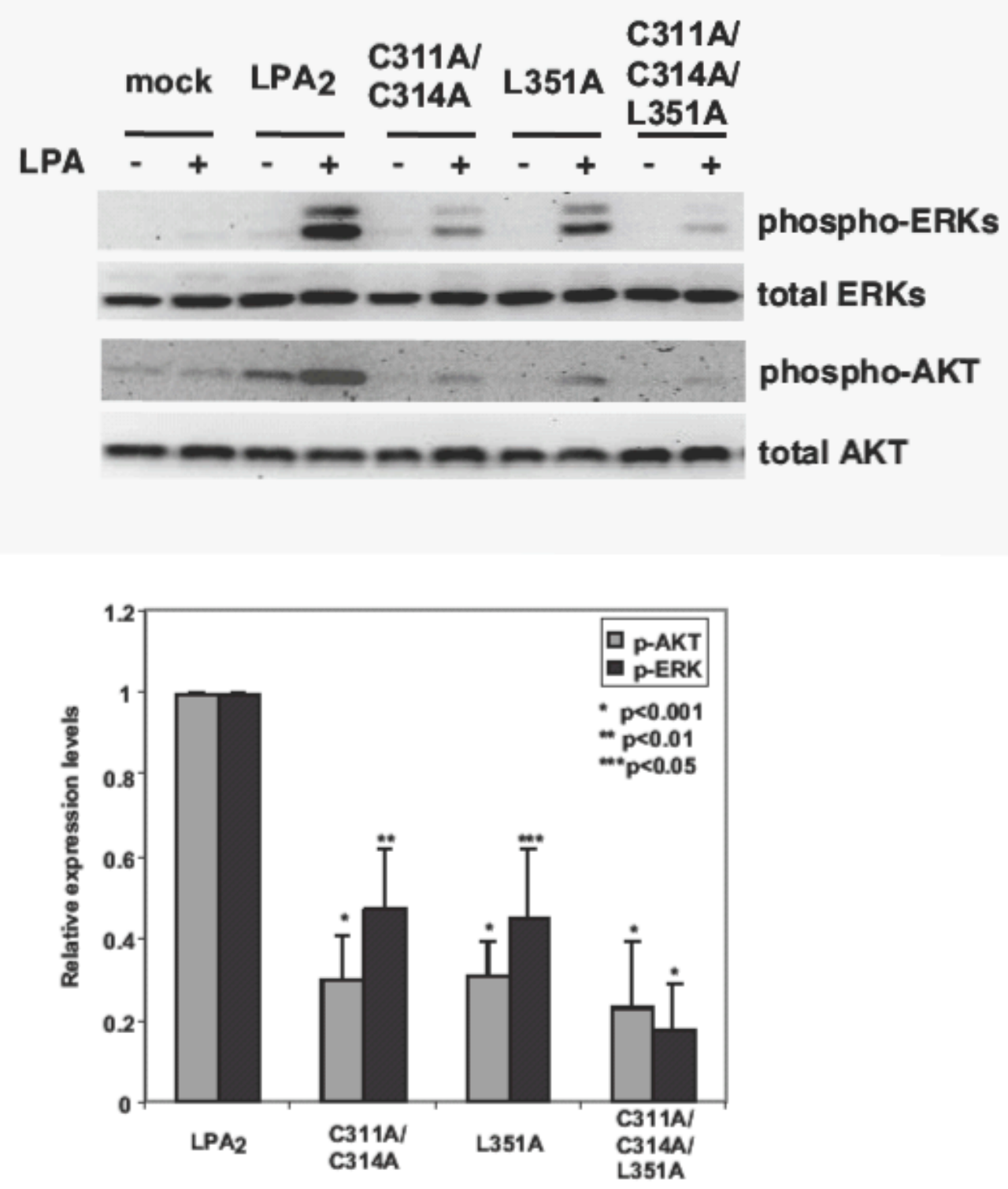

Figure 3.18. Mutation of $\mathrm{CxxC}$ and/or PDZ motif(s) of $\mathrm{LPA}_{2}-\mathrm{CT}$ attenuates ERK1/2 and Akt activation by LPA. The spontaneously immortalized DKO MEFs stably expressing GFP, WT LPA 2 , $\mathrm{LPA}_{2}-\mathrm{C} 311 \mathrm{~A} / \mathrm{C} 314 \mathrm{~A}, \mathrm{LPA}_{2}$-L351A or $\mathrm{LPA}_{2}-$ $\mathrm{C} 311 \mathrm{~A} / \mathrm{C} 314 \mathrm{~A} / \mathrm{L} 351 \mathrm{~A}$ and mock transduced by lentivirus were serum starved for $2.5 \mathrm{~h}$ in DMEM followed by stimulation with $1 \mu \mathrm{M}$ LPA for $10 \mathrm{~min}$. Cells were lyzed in SDS sample buffer and $15 \mu \mathrm{g}$ of the lysate was loaded for phospho-ERK1/2 and $80 \mu \mathrm{g}$ for phospho-Akt determination. The same blot was stripped and reprobed with mouse antiERK1 and rabbit anti-Akt IgG, respectively. 


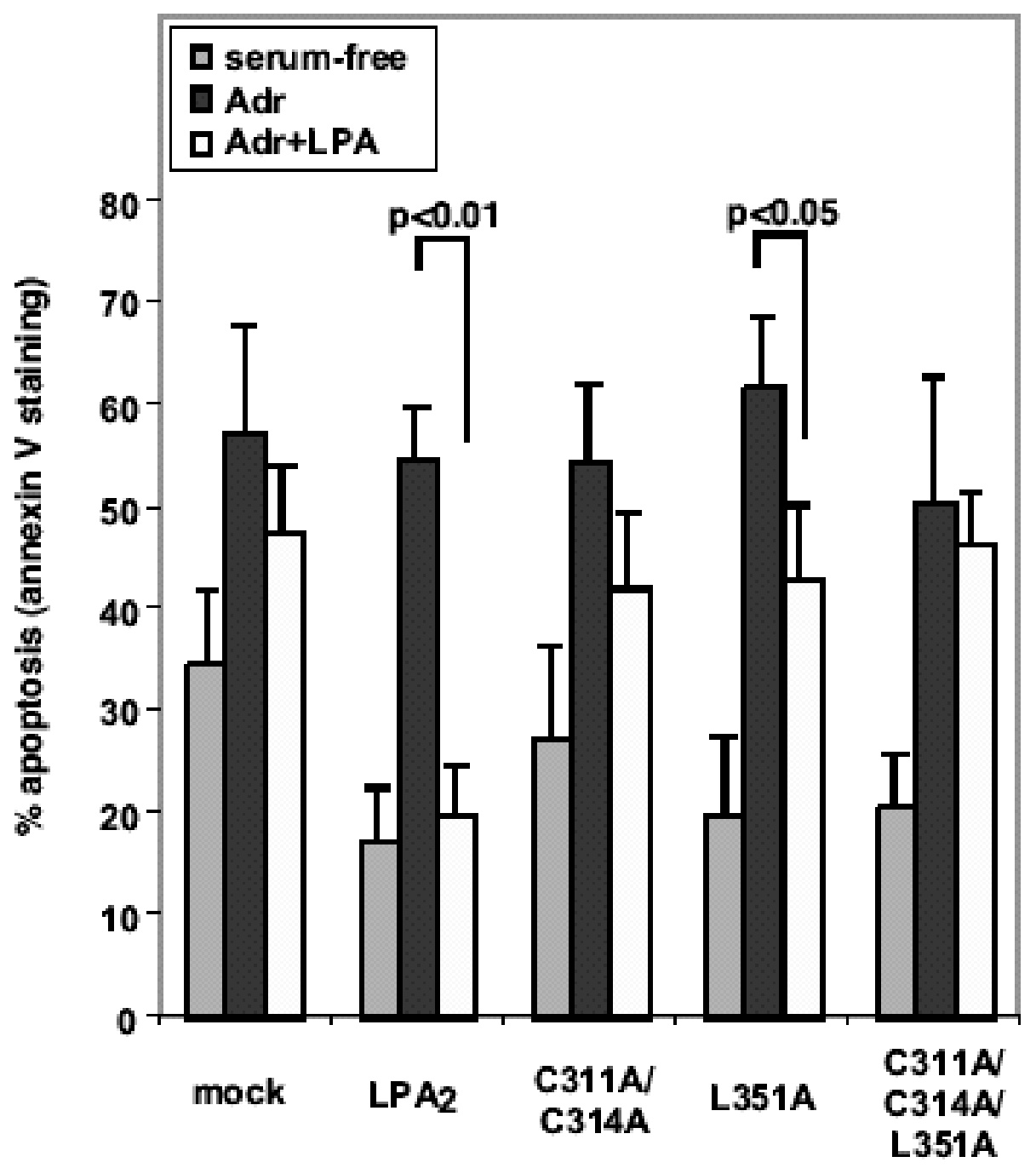

Figure 3.19. Mutation of $\mathrm{CxxC}$ and $\mathrm{PDZ}$ motifs of $\mathrm{LPA}-\mathrm{CT}$ abolishes protection against adriamycin-induced apoptosis revealed by Annexin $\mathrm{V}$ staining. Different MEF cell lines were pretreated with $10 \mu \mathrm{M}$ LPA in $0.1 \%$ BSA-containing medium for 1 or $2 \mathrm{~h}$ followed by the addition of $1.5 \mu \mathrm{M}$ adriamycin for $14 \mathrm{~h}$. Apoptosis was determined by Annexin V-FITC staining followed by flow cytometry analysis. Data shown is the mean \pm S.E.M. of three independent experiments. 


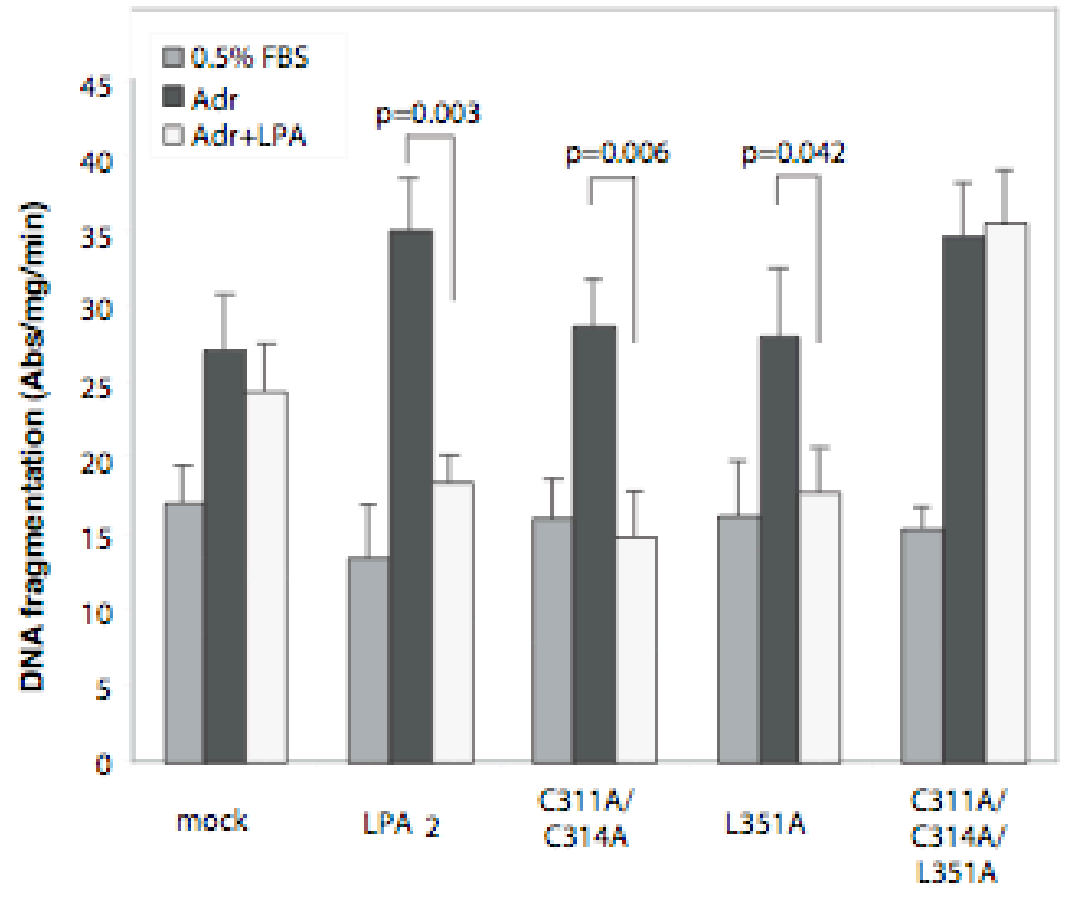

Figure 3.20. Mutation of $C x x C$ and PDZ motifs of $L P A_{2}-C T$ abolishes protection against adriamycin-induced apoptosis revealed by DNA fragmentation assay.

Different MEF cell lines were treated with $2 \mu \mathrm{M}$ adriamycin for $5.5 \mathrm{~h}$. Vehicle or $1 \mu \mathrm{M}$ LPA in $0.5 \%$ BSA-containing medium were treated $1 \mathrm{~h}$ after addition of adriamycin. Apoptosis was determined by DNA fragmentation assay as described in Materials and Methods. Data shown is the mean \pm S.E.M. of three independent experiments. 
mutants with disrupted interaction of Siva-1 with LPA 2 . To test this hypothesis, we examined procaspase 3 cleavage in these stable MEF lines challenged with $1.5 \mu \mathrm{M}$ adriamycin with or without $10 \mu \mathrm{M}$ LPA. LPA reversed procaspase 3 cleavage induced by adriamycin in the LPA 2 WT MEFs, which was not recapitulated in the mock, C311AC314A, and C311AC314AL351A expressing MEFs and much reduced in L351A MEFs, suggesting the role of both PDZ and LIM domain proteins in protecting from adriamycin-induced apoptosis (Fig. 3.21 top panel).

We also examined Siva-1 protein expression in these MEFs in response to LPA. In WT LPA 2 MEFs adriamycin-induced Siva-1 protein expression, which was attenuated by LPA treatment (Fig. 3.21 lower panel). In MEFs transduced with C311AC314A and C311AC314AL351A mutants LPA failed to reduce adriamycin-induced Siva-1 expression. In the L351A MEFs the reduction in Siva-1 expression was detected, indicating PDZ protein binding is not involved in regulating Siva-1 protein level.

\subsection{DISCUSSION}

LPA is a phospholipid growth factor that elicits cellular responses via the activation of its cognate GPCRs. Recently, a number of scaffold proteins interacting with $\mathrm{LPA}_{2} \mathrm{C}$-terminal tail $(\mathrm{CT})$ have been reported. These proteins mediate LPA signaling by protein-protein interactions different from the classical G-protein coupled pathways. The LIM domain proteins TRIP6 (Xu, 2004) and the proapoptotic molecule Siva-1 (Lin, 2007) represent a new set of interacting proteins whose binding site has not yet been identified. In contrast, the interaction of $\mathrm{LPA}_{2}-\mathrm{CT}$ with the PDZ domain proteins

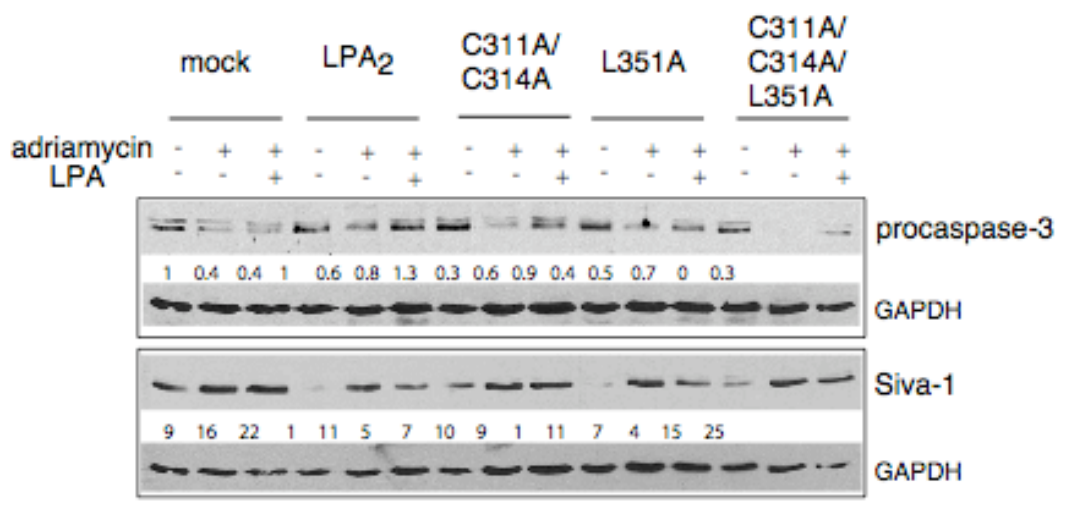

Figure 3.21. Mutation of CxxC and/or PDZ motif(s) of $\mathrm{LPA}_{2}-\mathrm{CT}$ abolishes protection against adriamycin-induced Caspase 3 activation and regulation of Siva1 expression. Different MEF cell lines were pretreated with $10 \mu \mathrm{M}$ LPA in $0.1 \%$ BSAcontaining medium for 1 or $2 \mathrm{~h}$ followed by the addition of $1.5 \mu \mathrm{M}$ adriamycin for $14 \mathrm{~h}$. The expression of procaspase- 3 and Siva- 1 was detected by immunoblotting using the antibodies specific to caspase-3 and Siva-1, respectively. 
including NHERF2, PDZ-RhoGEF, leukemia-associated RhoGEF, MAGI-3 (Oh, 2004; Wang, 2004; Yamada, 2005; Zhang, 2007) is much better understood in terms of the motifs that are required for this interaction as well as the physiological significance of the complex. Binding of LIM and PDZ domain proteins to the $\mathrm{LPA}_{2}-\mathrm{CT}$ also raises the question: is there competition or cooperativity between them?

Here we report the identification of a TRIP6 and Siva-1 binding motif in $\mathrm{LPA}_{2}$ CT: $\mathrm{C}^{311} \mathrm{xxC}^{314}$ (Fig.3.5 and Fig 3.9). Previously it has been shown that $\mathrm{LPA}_{2}-\mathrm{CT}$ interacts with aa278-476 constituting the third LIM domain TRIP6 (Xu, 2004) and the aa142-170 comprising the second zinc-finger domain of Siva-1 (Lin, 2007). The LIM domain consists of tandem zinc-finger motifs that are cysteine rich and capable of coordinating zinc (Dawid, 1998; Kadrmas \& Beckerle, 2004 review). Based on this criterion, Siva-1 can be relegated to the LIM domain proteins because it has two sequential zinc-finger motifs at its CT. Once considered to be exclusively a DNA-binding motif, zinc-finger now has been demonstrated to be a protein recognition motif as well (Mackay 1998; Gamsjaeger, 2007). The LIM/double zinc finger motif functions as a homo and heterodimerization domain (Feuerstein, 1994; Schmeichel \& Beckerle, 1994; Arber \& Caroni, 1996). So far no discrete consensus binding sequence has been identified for LIM domain proteins. LIM domains recognize different target proteins in a variable manner (Velyvis, 2003).

Although TRIP6 and Siva-1 bind to the same motif in $\mathrm{LPA}_{2}-\mathrm{CT}$, the binding affinity appears to be stronger for Siva-1 than TRIP6. One possible explanation is that there is one more cysteine in the second zinc-finger motif of Siva-1 than that in LIM3 of TRIP6. This may correlate with their different way of association with $\mathrm{LPA}_{2}$. Siva-1 binds to the internalized $\mathrm{LPA}_{2}$ and both end up in the proteasome for degradation, whereas the association of TRIP6 with $\mathrm{LPA}_{2}$ is transient since $\mathrm{LPA}_{2}$ is not at the focal adhesion plaque where TRIP6 localizes after LPA stimulation for 15-20 min (Xu, 2004; Lin, 2007; Lin, 2008 review). Intriguingly, the other EDG family receptors whose ligand is sphingosine-1-phosphate, $\mathrm{S}_{1} \mathrm{P}_{1}$ and $\mathrm{S}_{1} \mathrm{P}_{4}$ also contain the $\mathrm{CxxC}$ motifs at their CTs. Whether these receptors interact with LIM proteins remains to be determined.

Cysteine residues in the C-termini of GPCR are targets for posttranslational modification by palmitoylation. Palmitoylation of the GPCRs plays important roles in receptor binding, targeting, trafficking, signaling and protein interactions (Qanbar \& Bouvier, 2003 review; Resh, 2006 review; Escriba, 2007 review). In addition, palmitoylation of cys 261 in the Hepatitis C virus NS4B protein is important for the protein-protein interaction in the formation of the virus RNA replication complex $(\mathrm{Yu}$, 2006). Palmitoylation is required for postsynaptic density (PSD-95) protein multidimerization, postsynaptic targeting and clustering of glutamate receptors (Christopherson, 2003). We identified multiple palmitoylation sites in the $\mathrm{LPA}_{2}-\mathrm{CT}$ that included the CxxC motif. This raised the possibility that palmitoylation of these two cysteine residues involved in the interaction with LIM proteins might regulate their interaction. Our pull down experiments with recombinant $\mathrm{LPA}_{2}-\mathrm{CT}$ and the Siva-1 or TRIP6 showed that palmitoylation is not required for complex formation because E. Coli lacks Protein Acyl Transferases (PATs). However, when transfected into HEK-293 cells, 
we found that the CxxC motif was palmitoylated but blocking palmitoylation of $\mathrm{LPA}_{2}$ did not affect its association with TRIP6 or Siva-1. Using the acyl-biotinyl exchange method we have also determined that in HEK293 cells TRIP6 forms a complex with palmitoylated $\mathrm{CxxC}$ motifs. In this procedure, the palmitoyl-cysteine is specificly biotinylated using hydroxylamine and biotin-HPDP. Coimmunoprecipitation of the TRIP6-LPA 2 complex showed that the $\mathrm{LPA}_{2}$ present in this complex was palmitoylated because it was labeled with biotin during the acyl-biotin exchange chemistry (Fig. 3.12). These observations indicate that palmitoylation is not required for the interaction of the LIM domain protein TRIP6 and LPA 2 , discounting the role of palmitoylation-mediated regulation of their interacion.

Previously we reported that $\mathrm{LPA}_{2}$ reconstitution into RH cells devoid of EDG family LPA receptors, but not $\mathrm{LPA}_{1}$ or $\mathrm{LPA}_{3}$, was capable of rescuing these cells from TNF $\alpha$-induced apoptosis by OTP. In support of the essential role of $\mathrm{LPA}_{2}$, neither LPA nor OTP protected the intestinal mucosa of $\mathrm{LPA}_{2}$ knockout mice, whereas both ligands were active in $\mathrm{LPA}_{1}$ receptor knockouts $(\mathrm{E}, 2007)$. The activation of ERK and protective effect of OTP in $\mathrm{LPA}_{2}$-reconstituted RH that is partially sensitive to PTX indicate a role for non- $\mathrm{G}_{\mathrm{i}}$ mediated signals. The antiapoptotic effect of OTP in $\mathrm{LPA}_{2}$-reconstituted $\mathrm{RH}$ cells could not be explained by $\mathrm{G}_{\mathrm{q}}$ mediated signals because all three LPA receptors activate $\mathrm{G}_{\mathrm{q}}$-linked $\mathrm{Ca}^{2+}$ transients, yet only $\mathrm{LPA}_{2}$ was protective. In support of this, Yun and colleagues found that LPA protects Caco 2 cells, which predominately express $\mathrm{LPA}_{2}$, from etopside-induced apoptosis by activation of ERK and inactivation of Bad and caspase 3 in a PTX-insensitive, NHERF2-dependent manner (Yun, 2005; Rusovici, 2007). These observations suggest $G$ protein-independent signaling is involved in $\mathrm{LPA}_{2}$ mediated protection against apoptosis. We hypothesized that the protein-protein interactions mediated by the $\mathrm{LPA}_{2}-\mathrm{CT}$ play a role in this cellular response.

Knockdown of the PDZ binding protein NHERF2 attenuates LPA-induced ERK and Akt and abrogates the protective effect of $\mathrm{LPA}_{2}$ in Caco-2 cells (Yun, 2005; Rusovici, 2007). MAGI-3 regulates LPA-dependent ERK and RhoA activation via interaction with $\mathrm{LPA}_{2}$ (Zhang, 2007). These reports taken together implicate the role of protein-protein interactions mediated via the PDZ interaction motif of $\mathrm{LPA}_{2}$ in antiapoptotic signaling. Similarly, LPA-induced ERK activation is strongly reduced by siRNA-mediated knockdown of TRIP6 (Lai, 2005), suggesting a role for a LIM protein in antiapoptotic signaling. Lai and colleagues showed that c-Src phosphorylates TRIP6 at Tyr55 and this phosphorylation enhances LPA-induced ERK activation. Knockdown of TRIP6 drastically attenuates LPA-induced ERK1/2 activation in c-Src expressing cells (Lai, 2005). In support of this, LPA activates c-Src (Luttrell, 1996; Kranenburg, 1997), and c-Src activity is required for the antiapoptotic effect of LPA and OTP (Deng \& Tigyi - unpublished). LPA $_{2}$ interacts with the proapoptotic transcription factor Siva-1 and LPA stimulation promotes the association of Siva-1 with $\mathrm{LPA}_{2}$ and targets the Siva-1-LPA complex for ubiquitination and degradation (Lin, 2007). Siva-1 has been reported to form a complex with the antiapoptotic protein $\mathrm{Bcl}-\mathrm{X}_{\mathrm{L}}$, reducing its availability to protect mitochondrial outer membrane integrity (Xue, 2002; Chu, 2004). We found, in IEC-6 (intestinal epithelial cells) and also in mice treated with OTP, that Bcl- $\mathrm{X}_{\mathrm{L}}$ levels did not 
go down but increase (E, 2007). This could be due to $\mathrm{LPA}_{2}$-mediated degradation of Siva-1, which in turn would allow Bcl- $\mathrm{X}_{\mathrm{L}}$ to exert its protective action.

Our results using $\mathrm{LPA}_{2}$ alanine mutants defective in interaction with either PDZ domain binding proteins (L351A) or with LIM domain proteins (C311AC314A) attenuated ERK and Akt phosphorylation by LPA in MEFs. Mutation of both domains in the C311AC314AL351A construct completely abolished the activation of ERK and Akt in MEFs (Fig.3.18). Consistent with this, the anti-apoptotic effect of LPA on adriamycininduced apoptosis, Siva-1 expression and procaspase 3 cleavage were severely compromised in the C311AC314AL351A mutant (Fig.3.19, Fig.3.20 and Fig.3.21). The L351A or C311AC314A mutant showed reduced protection that paralleled their attenuated activation of the LPA 2 coupled prosurvival pathways. These results suggest cooperativity of PDZ and LIM proteins in mediating the LPA $\mathrm{L}_{2}$ signaling. How is this cooperativity achieved? Our experiments showed that in the case of TRIP6, mutation of L351 to alanine reduced complex formation (Fig.3.3). PDZ domain binding proteins such as NHERF2 possess an $\mathrm{N}$ - and also a $\mathrm{C}$-terminal PDZ binding domains. TRIP6, like $\mathrm{LPA}_{2}$, contains a C-terminal PDZ interaction motif. Taken together, the possibility exists that the complex of LPA 2 with TRIP-6 is stabilized via a NHERF2 bridge engaging the PDZ motifs. Moreover, the ability of homodimerization of $\mathrm{LPA}_{2}$ and binding to TRIP6 and/or Siva-1 provides much more flexibility for multi-protein complex formation (Bouvier, 2001). It has been proposed that proteins containing multiple LIM domains might function as a molecular bridge (Kadrmas \& Beckerle, 2004 review). More work needs to be done to test this hypothesis and reveal the downstream signaling events initiated by protein-protein interaction with $\mathrm{LPA}_{2}$.

Altogether, in this work we provide direct evidence, by using stable MEFs expressing comparable levels of $\mathrm{LPA}_{2} \mathrm{WT}$ and mutants, that the protein-protein interaction between $\mathrm{LPA}_{2}$-CT with PDZ/TRIP6/Siva-1 mediates anti-apoptotic effects of LPA $_{2}$ (Fig. 3.19, Fig.3.20, and Fig.3.21). The mutants defective in their interaction with LIM and PDZ binding proteins remain fully capable of activating $G$ protein-coupled signals such as $\mathrm{Ca}^{2+}$ mobilization (Fig.3.17). The present findings expand the traditional view of G-protein coupling-mediated signaling of GPCRs and link it to direct pathways regulating a transcription factor Siva-1 and a scaffold protein TRIP6 (Fig. 3.22). Recently, inhibition of the protein-protein interaction with small-molecules is becoming an emerging area in drug discovery (Fry, 2006 \& 2008 review). Hence, advances in understanding protein-protein interactions with $\mathrm{LPA}_{2}$ may provide the bases for discovering drugs that have therapeutic potential. 


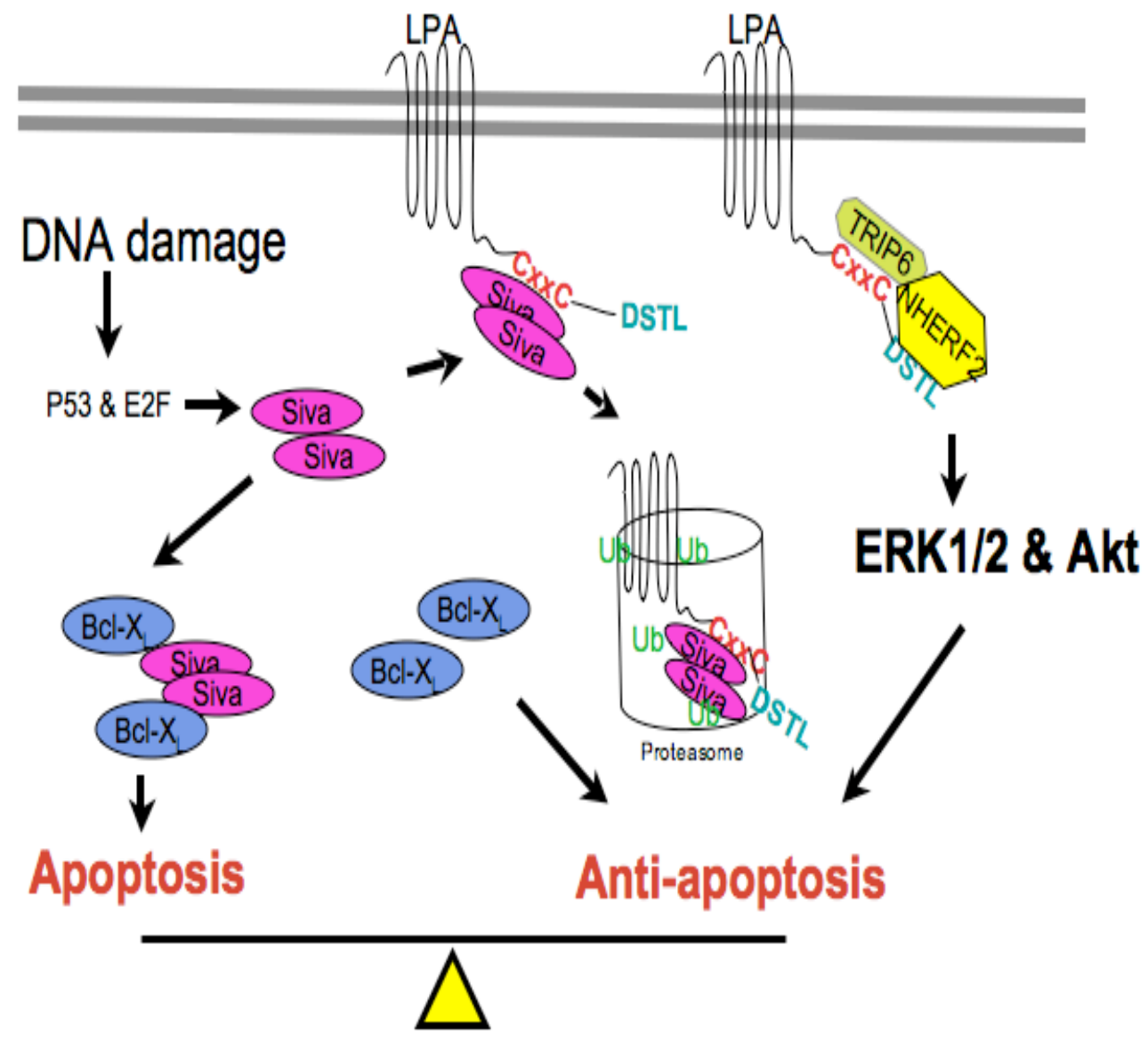

Figure 3.22. Scheme of antiapoptotic mechanism of $\mathrm{LPA}_{2}$ by protein-protein interaction. DNA damage induces proapoptotic molecule Siva-1 expression and Siva-1 binds to antiapoptotic protein Bcl- $\mathrm{X}_{\mathrm{L}}$ to induce apoptosis. Upon LPA stimulation, Siva-1, associating with $\mathrm{LPA}_{2}$ by $\mathrm{CxxC}$ motif, is targeted to proteasome for degradation, thereby no longer available to bind up Bcl- $\mathrm{X}_{\mathrm{L}}$. In addition, TRIP6 and NHERF2 are recruited to the $\mathrm{LPA}_{2}$ by binding to $\mathrm{CxxC}$ and DSTL motifs, respectively, with LPA stimulation and NHERF2 also binds to TRIP6 via TTDC motif, forming a macromolecular complex. ERKs and Akt prosurvival pathways are activated and mediate the antiapoptotic effect. 


\section{LIST OF REFERENCES}

Anliker B, Chun J. Cell surface receptors in lysophospholipid signaling. Semin Cell Dev Biol. 2004 Oct; 15(5):457-65. Review.

Aoki J. Mechanisms of lysophosphatidic acid production. Semin Cell Dev Biol. 2004 Oct; 15(5):477-89. Review.

Arber S, Caroni P. Specificity of single LIM motifs in targeting and LIM/LIM interactions in situ. Genes Dev. 1996 Feb 1; 10(3):289-300.

Bach I. The LIM domain: regulation by association. Mech Dev. 2000 91(1-2): 5-17.

Balazs L, Okolicany J, Ferrebee M, Tolley B, Tigyi G. Topical application of the phospholipid growth factor lysophosphatidic acid promotes wound healing in vivo. Am J Physiol Regul Integr Comp Physiol. 2001 280:R466-72.

Ballesteros JA, Weinstein H. Chapter 19. In Conn PM, Sealfon SC, eds. Methods in Neurosciences. San Diego: Academic Press, 1995: 366-428.

Beckerle MC. Zyxin: zinc fingers at sites of cell adhesion. Bioessays. 1997 Nov; 19(11):949-57. Review.

Brady AE, Limbird LE. G protein-coupled receptor interacting proteins: emerging roles in localization and signal transduction. Cell Signal. 2002 Apr; 14(4):297-309. Review.

Bras M, Queenan B, Susin SA. Programmed cell death via mitochondria: different modes of dying. Biochem (Mosc). 2005 Feb; 70(2):231-9. Review.

Brindley DN, English D, Pilquil C, Buri K, Ling ZC. Lipid phosphate phosphatases regulate signal transduction through glycerolipids and sphingolipids. Biochim Biophys Acta. 2002 1582:33-44.

Chevreau N, Funk-Archuleta M. Effect of enteral formulas on methotrexate toxicity. Nutr Cancer. 1995 23:185-204.

Chipuk JE, Green DR. Dissecting p53-dependent apoptosis. Cell Death Differ. 2006 Jun; 13(6):994-1002. Review.

Chou CH, Wei LH, Kuo ML, Huang YJ, Lai KP, Chen CA, Hsieh CY. Up-regulation of interleukin-6 in human ovarian cancer cell via a Gi/PI3K-Akt/NF-kappaB pathway by lysophosphatidic acid, an ovarian cancer-activating factor. Carcinogenesis. 2005 26:4552.

Chowdhury I, Tharakan B, Bhat GK. Current concepts in apoptosis: the physiological suicide program revisited. Cell Mol Biol Lett. 2006 11(4):506-25. 
Christopherson KS, Sweeney NT, Craven SE, Kang R, El-Husseini Ael-D, Bredt DS. Lipid- and protein-mediated multimerization of PSD-95: implications for receptor clustering and assembly of synaptic protein networks. J Cell Sci. 2003 Aug 1; 116(Pt 15):3213-9.

Chu F, Barkinge J, Hawkins S, Gudi R, Salgia R, Kanteti PV. Expression of Siva-1 protein or its putative amphipathic helical region enhances cisplatin-induced apoptosis in breast cancer cells: effect of elevated levels of BCL-2. Cancer Res. 2005 Jun 15; 65(12):5301-9.

Chu F, Borthakur A, Sun X, Barkinge J, Gudi R, Hawkins S, Prasad KV. The Siva-1 putative amphipathic helical region (SAH) is sufficient to bind to BCL-XL and sensitize cells to UV radiation induced apoptosis. Apoptosis. 2004 Jan; 9(1):83-95.

Contos JJ, Fukushima N, Weiner JA, Kaushal D, Chun J. Requirement for the LPA1 lysophosphatidic acid receptor gene in normal suckling behavior. Proc Natl Acad Sci U S A. 2000 Nov 21; 97(24):13384-9.

Contos JJ, Ishii I, Fukushima N, Kingsbury MA, Ye X, Kawamura S, Brown JH, Chun J. Characterization of $\operatorname{lpa}(2)(\mathrm{Edg} 4)$ and $\operatorname{lpa}(1) / \operatorname{lpa}(2)(\mathrm{Edg} 2 / \mathrm{Edg} 4)$ lysophosphatidic acid receptor knockout mice: signaling deficits without obvious phenotypic abnormality attributable to lpa(2). Mol Cell Biol. 2002 Oct; 22(19):6921-9.

Cuppen E, van Ham M, Wansink DG, de Leeuw A, Wieringa B, Hendriks W. The zyxinrelated protein TRIP6 interacts with PDZ motifs in the adaptor protein RIL and the protein tyrosine phosphatase PTP-BL. Eur J Cell Biol. 2000 79(4): 283-93.

Danial NN, Korsmeyer SJ. Cell death: critical control points. Cell. 2004 Jan 23; 116(2):205-19. Review.

Daoud SS, Munson PJ, Reinhold W, Young L, Prabhu VV, Yu Q, LaRose J, Kohn KW, Weinstein JN, Pommier Y. Impact of p53 knockout and topotecan treatment on gene expression profiles in human colon carcinoma cells: a pharmacogenomic study. Cancer Res. 2003 Jun 1; 63(11):2782-93.

Dawid IB, Breen JJ, Toyama R. LIM domains: multiple roles as adapters and functional modifiers in protein interactions. Trends Genet. 1998 Apr; 14(4):156-62. Review.

Deng W, Balazs L, Wang DA, Van Middlesworth L, Tigyi G, Johnson LR. Lysophosphatidic acid protects and rescues intestinal epithelial cells from radiation- and chemotherapy-induced apoptosis. Gastroenterology. 2002 Jul; 123(1):206-16.

Deng W, Poppleton H, Yasuda S, Makarova N, Shinozuka Y, Wang DA, Johnson LR, Patel TB, Tigyi G. Optimal lysophosphatidic acid-induced DNA synthesis and cell migration but not survival require intact autophosphorylation sites of the epidermal growth factor receptor. J Biol Chem. 2004 Nov 12; 279(46):47871-80. 
Deng W, Wang DA, Gosmanova E, Johnson LR, Tigyi G. LPA protects intestinal epithelial cells from apoptosis by inhibiting the mitochondrial pathway. Am J Physiol Gastrointest Liver Physiol. 2003 May; 284(5):G821-9.

Dunphy JT, Linder ME. Signalling functions of protein palmitoylation. Biochim Biophys Acta. 1998 Dec 8; 1436(1-2):245-61. Review.

Durgam GG, Virag T, Walker MD, Tsukahara R, Yasuda S, Liliom K, van Meeteren LA, Moolenaar WH, Wilke N, Siess W, Tigyi G, Miller DD. Synthesis, structure-activity relationships, and biological evaluation of fatty alcohol phosphates as lysophosphatidic acid receptor ligands, activators of PPARgamma, and inhibitors of autotaxin. J Med Chem. 2005 Jul 28; 48(15):4919-30.

E S, Deng W, Tsukahara R, Valentine WJ, Durgam G, Gududuru V, Balazs L, Manickam V, Arsura M, VanMiddlesworth L, Johnson LR, Parrill AL, Miller DD, Tigyi G. The lysophosphatidic acid type 2 receptor is required for protection against radiation-induced intestinal injury. Gastroenterology. 2007 May; 132(5):1834-51.

Erster S, Mihara M, Kim RH, Petrenko O, Moll UM. In vivo mitochondrial p53 translocation triggers a rapid first wave of cell death in response to DNA damage that can precede p53 target gene activation. Mol Cell Biol. 2004 Aug; 24(15):6728-41.

Escribá PV, Wedegaertner PB, Goñi FM, Vögler O. Lipid-protein interactions in GPCRassociated signaling. Biochim Biophys Acta. 2007 Apr; 1768(4):836-52. Review.

Fang X, Yu S, Bast RC, Liu S, Xu HJ, Hu SX, LaPushin R, Claret FX, Aggarwal BB, Lu Y, Mills GB. Mechanisms for lysophosphatidic acid-induced cytokine production in ovarian cancer cells. J Biol Chem. 2004 Mar 5; 279(10):9653-61.

Fang X, Yu S, Eder A, Mao M, Bast RC Jr, Boyd D, Mills GB. Regulation of BAD phosphorylation at serine 112 by the Ras-mitogen-activated protein kinase pathway. Oncogene. 1999 18(48): 6635-40.

Fang X, Yu S, LaPushin R, Lu Y, Furui T, Penn LZ, Stokoe D, Erickson JR, Bast RC Jr, Mills GB. Lysophosphatidic acid prevents apoptosis in fibroblasts via G(i)-proteinmediated activation of mitogen-activated protein kinase. Biochem J. 2000 Nov 15; 352 Pt $1: 135-43$.

Fei P, El-Deiry WS. p53 and radiation responses. Oncogene. 2003 Sep 1; 22(37):577483. Review.

Feuerstein R, Wang X, Song D, Cooke NE, Liebhaber SA.The LIM/double zinc-finger motif functions as a protein dimerization domain. Proc Natl Acad Sci U S A. 1994 Oct 25; 91(22):10655-9. 
Fischer DJ, Liliom K, Guo Z, Nusser N, Virag T, Murakami-Murofushi K, Kobayashi S, Erickson JR, Sun G, Miller DD, Tigyi G. Naturally occurring analogs of lysophosphatidic acid elicit different cellular responses through selective activation of multiple receptor subtypes. Mol Pharmacol. 1998 54:979-88.

Fischer DJ, Nusser N, Virag T, Yokoyama K, Wang D, Baker DL, Bautista D, Parrill AL, Tigyi G. Short-chain phosphatidates are subtype-selective antagonists of lysophosphatidic acid receptors. Mol Pharmacol. 2001 60:776-84.

Fortin A, MacLaurin JG, Arbour N, Cregan SP, Kushwaha N, Callaghan SM, Park DS, Albert PR, Slack RS. The proapoptotic gene SIVA is a direct transcriptional target for the tumor suppressors p53 and E2F1. J Biol Chem. 2004 Jul 2; 279(27):28706-14.

Frankel A, Mills GB. Peptide and lipid growth factors decrease cisdiamminedichloroplatinum-induced cell death in human ovarian cancer cells. Clin Cancer Res. 1996 Aug; 2(8):1307-13.

Fry DC. Drug-like inhibitors of protein-protein interactions: a structural examination of effective protein mimicry. Curr Protein Pept Sci. 2008 Jun; 9(3):240-7.

Fry DC. Protein-protein interactions as targets for small molecule drug discovery. Biopolymers. 2006 84(6):535-52. Review.

Fujiwara Y, Sardar V, Tokumura A, Baker DL, Murakami-Murofushi K, Parrill AL, Tigyi, G.. Identification of residues responsible for ligand recognition and regioisomeric selectivity of LPA receptors expressed in mammalian cells. J Biol Chem. 2005 280:35038-50.

Funk-Archuleta MA, Foehr MW, Tomei LD, Hennebold KL, Bathurst IC. A soy-derived antiapoptotic fraction decreases methotrexate toxicity in the gastrointestinal tract of the rat. Nutr Cancer. 1997 29:217-21.

Gamsjaeger R, Liew CK, Loughlin FE, Crossley M, Mackay JP. Sticky fingers: zincfingers as protein-recognition motifs. Trends Biochem Sci. 2007 Feb; 32(2):63-70.

Goetzl EJ, Lee H, Dolezalova H, Kalli KR, Conover CA, Hu YL, Azuma T, Stossel TP, Karliner JS, Jaffe RB. Mechanisms of lysolipid phosphate effects on cellular survival and proliferation. Ann N Y Acad Sci. 2000 Apr; 905:177-87. Review.

Green DR. At the gates of death. Cancer Cell. 2006 May; 9(5):328-30.

Gudi R, Barkinge J, Hawkins S, Chu F, Manicassamy S, Sun Z, Duke-Cohan JS, Prasad KV. Siva-1 negatively regulates NF-kappaB activity: effect on T-cell receptor-mediated activation-induced cell death (AICD). Oncogene. 2006 25:3458-62.

Halgren TA. Merck molecular force field. I. Basis, form, scope, parameterization, and performance of MMFF94*. J Comp Chem. 1996 17:490-519. 
Hall PA, Coates PJ, Ansari B, Hopwood D. Regulation of cell number in the mammalian gastrointestinal tract: the importance of apoptosis. J Cell Sci. 1994107 (Pt 12):3569-77.

Hall RA, Lefkowitz RJ. Regulation of G protein-coupled receptor signaling by scaffold proteins. Circ Res. 2002 Oct 18; 91(8):672-80. Review.

Hall RA, Ostedgaard LS, Premont RT, Blitzer JT, Rahman N, Welsh MJ, Lefkowitz RJ. A C-terminal motif found in the beta2-adrenergic receptor, $\mathrm{P} 2 \mathrm{Y} 1$ receptor and cystic fibrosis transmembrane conductance regulator determines binding to the $\mathrm{Na}+/ \mathrm{H}+$ exchanger regulatory factor family of PDZ proteins. Proc Natl Acad Sci U S A. 1998 Jul $21 ; 95(15): 8496-501$.

Harris BZ, Lim WA. Mechanism and role of PDZ domains in signaling complex assembly. J Cell Sci. 2001 114(Pt 18): 3219-31.

Harris SL, Levine AJ. The p53 pathway: positive and negative feedback loops. Oncogene. 2005 Apr 18; 24(17):2899-908. Review.

Hickman ES, Moroni MC, Helin K. The role of p53 and pRB in apoptosis and cancer. Curr Opin Genet Dev. 2002 Feb; 12(1):60-6. Review.

Hines OJ, Ryder N, Chu J, McFadden D. Lysophosphatidic acid stimulates intestinal restitution via cytoskeletal activation and remodeling. J Surg Res. 2000 92:23-8.

Hu X, Haney N, Kropp D, Kabore AF, Johnston JB, Gibson SB. Lysophosphatidic acid (LPA) protects primary chronic lymphocytic leukemia cells from apoptosis through LPA receptor activation of the anti-apoptotic protein AKT/PKB. J Biol Chem. 2005 Mar 11; 280(10):9498-508.

Hung AY, Sheng M. PDZ domains: structural modules for protein complex assembly. J Biol Chem. 2002 Feb 22; 277(8):5699-702.

Inagaki Y, Pham TT, Fujiwara Y, Kohno T, Osborne DA, Igarashi Y, Tigyi G, Parrill AL.. Sphingosine 1-phosphate analogue recognition and selectivity at S1P4 within the endothelial differentiation gene family of receptors. Biochem J. 2005 389:187-95.

Ishii I, Contos JJ, Fukushima N, Chun J. Functional comparisons of the lysophosphatidic acid receptors, LP(A1)/VZG-1/EDG-2, LP(A2)/EDG-4, and LP(A3)/EDG-7 in neuronal cell lines using a retrovirus expression system. Mol Pharmacol. 2000 Nov; 58(5):895902.

Ishii I, Fukushima N, Ye X, Chun J. Lysophospholipid receptors: signaling and biology. Annu Rev Biochem. 2004 73:321-54. Review.

Jacobs SB, Basak S, Murray JI, Pathak N, Attardi LD. Siva is an apoptosis-selective p53 target gene important for neuronal cell death. Cell Death Differ. 2007 Jul; 14(7):1374-85. 
Jeleń F, Oleksy A, Smietana K, Otlewski J. PDZ domains - common players in the cell signaling. Acta Biochim Pol. 2003 50(4):985-1017. Review.

Jin Z, El-Deiry WS. Overview of cell death signaling pathways. Cancer Biol Ther. 2005 Feb; 4(2):139-63. Review.

Jo E, Sanna MG, Gonzalez-Cabrera PJ, Thangada S, Tigyi G, Osborne DA, Hla T, Parrill AL, Rosen H. S1P1-selective in vivo-active agonists from high-throughput screening: off-the-shelf chemical probes of receptor interactions, signaling, and fate. Chem Biol. 2005 12:703-15.

Kadrmas JL, Beckerle MC. The LIM domain: from the cytoskeleton to the nucleus. Nat Rev Mol Cell Biol. 2004 Nov; 5(11):920-31. Review.

Kang YC, Kim KM, Lee KS, Namkoong S, Lee SJ, Han JA, Jeoung D, Ha KS, Kwon YG, Kim YM. Serum bioactive lysophospholipids prevent TRAIL-induced apoptosis via PI3K/Akt-dependent cFLIP expression and Bad phosphorylation. Cell Death Differ. 2004 11(12): 1287-98.

Kates M. Techniques of Lipidology. 1988 Amsterdam, New York, Oxford: Elsevier.

Koh JS, Lieberthal W, Heydrick S, Levine JS. Lysophosphatidic acid is a major serum noncytokine survival factor for murine macrophages which acts via the phosphatidylinositol 3-kinase signaling pathway. J Clin Invest. 1998 Aug 15; 102(4):71627.

Kotarsky K, Boketoft A, Bristulf J, Nilsson NE, Norberg A, Hansson S, Owman C, Sillard R, Leeb-Lundberg LM, Olde B. Lysophosphatidic acid binds to and activates GPR92, a G protein-coupled receptor highly expressed in gastrointestinal lymphocytes. J Pharmacol Exp Ther. 2006 Aug; 318(2):619-28.

Kranenburg O, Verlaan I, Hordijk PL, Moolenaar WH. Gi-mediated activation of the Ras/MAP kinase pathway involves a $100 \mathrm{kDa}$ tyrosine-phosphorylated Grb2 SH3 binding protein, but not Src nor Shc. EMBO J. 1997 Jun 2; 16(11):3097-105.

Lai YJ, Chen CS, Lin WC, Lin FT. c-Src-mediated phosphorylation of TRIP6 regulates its function in lysophosphatidic acid-induced cell migration. Mol Cell Biol. $2005 \mathrm{Jul}$; 25(14):5859-68.

Lai YJ, Lin WC, Lin FT. PTPL1/FAP-1 negatively regulates TRIP6 function in lysophosphatidic acid-induced cell migration. J Biol Chem. 2007 Aug 17; 282(33):24381-7.

Lee CW, Rivera R, Dubin AE, Chun J. LPA(4)/GPR23 is a lysophosphatidic acid (LPA) receptor utilizing $\mathrm{G}(\mathrm{s})-, \mathrm{G}(\mathrm{q}) / \mathrm{G}(\mathrm{i})$-mediated calcium signaling and $\mathrm{G}(12 / 13)$-mediated Rho activation. J Biol Chem. 2007 Feb 16; 282(7):4310-7. 
Lee CW, Rivera R, Gardell S, Dubin AE, Chun J. GPR92 as a new G12/13 and Gq coupled lysophosphatidic acid receptor that increases cAMP: LPA5. J Biol Chem. 2006 Aug 18; 281(33):23589-97.

Lee JW, Choi HS, Gyuris J, Brent R, Moore DD. Two classes of proteins dependent on either the presence or absence of thyroid hormone for interaction with the thyroid hormone receptor. Mol Endocrinol. 1995 Feb; 9(2):243-54.

Lefkowitz RJ. Seven transmembrane receptors: something old, something new. Acta Physiol (Oxf). 2007 May; 190(1):9-19. Review.

Lefkowitz RJ, Rajagopal K, Whalen EJ. New roles for beta-arrestins in cell signaling: not just for seven-transmembrane receptors. Mol Cell. 2006 Dec 8; 24(5):643-52. Review

Levine JS, Koh JS, Triaca V, Lieberthal W. Lysophosphatidic acid: a novel growth and survival factor for renal proximal tubular cells. Am J Physiol. 1997 Oct; 273(4 Pt 2):F575-85.

Li C, Dandridge KS, Di A, Marrs KL, Harris EL, Roy K, Jackson JS, Makarova NV, Fujiwara Y, Farrar PL, Nelson DJ, Tigyi GJ, Naren AP. Lysophosphatidic acid inhibits cholera toxin-induced secretory diarrhea through CFTR-dependent protein interactions. J Exp Med. 2005a Oct 3; 202(7):975-86.

Li L, Bin LH, Li F, Liu Y, Chen D, Zhai Z, Shu HB. TRIP6 is a RIP2-associated common signaling component of multiple NF-kappaB activation pathways. J Cell Sci. 2005b Feb 1; 118(Pt 3):555-63.

Li Y, Gonzalez MI, Meinkoth JL, Field J, Kazanietz MG, Tennekoon GI. Lysophosphatidic acid promotes survival and differentiation of rat Schwann cells. J Biol Chem. 2003 Mar 14; 278(11):9585-91.

Lin FT, Lai YJ. Regulation of the LPA(2) receptor signaling through the carboxylterminal tail-mediated protein-protein interactions. Biochim Biophys Acta. 2008 Sep; 1781(9):558-62.

Lin FT, Lai YJ, Makarova N, Tigyi G, Lin WC. The lysophosphatidic acid 2 receptor mediates down-regulation of Siva-1 to promote cell survival. J Biol Chem. 2007 Dec 28; 282(52):37759-69.

Logvinova AV, Foehr MW, Pemberton PA, Khazalpour KM, Funk-Archuleta MA, Bathurst IC, Tomei LD. Soy-derived antiapoptotic fractions protect gastrointestinal epithelium from damage caused by methotrexate treatment in the rat. Nutr Cancer. 1999 33:33-9. 
Luttrell LM. Composition and function of g protein-coupled receptor signalsomes controlling mitogen-activated protein kinase activity. J Mol Neurosci. 2005 26(2-3):25364. Review.

Luttrell LM, Hawes BE, van Biesen T, Luttrell DK, Lansing TJ, Lefkowitz RJ. Role of cSrc tyrosine kinase in G protein-coupled receptor- and Gbetagamma subunit-mediated activation of mitogen-activated protein kinases. J Biol Chem. 1996 Aug 9; 271(32):19443-50.

Macalma T, Otte J, Hensler ME, Bockholt SM, Louis HA, Kalff-Suske M, Grzeschik $\mathrm{KH}$, von der Ahe D, Beckerle MC. Molecular characterization of human zyxin.

J Biol Chem. 1996 Dec 6; 271(49):31470-8.

Mackay JP, Crossley M. Zinc fingers are sticking together. Trends Biochem Sci. 1998 Jan; 23(1):1-4. Review.

Marrero MB, Venema VJ, Ju H, Eaton DC, Venema RC. Regulation of angiotensin IIinduced JAK2 tyrosine phosphorylation: roles of SHP-1 and SHP-2. Am J Physiol. 1998 Nov; 275(5 Pt 1):C1216-23.

Marshman E, Booth C, Potten CS. The intestinal epithelial stem cell. Bioessays. 2002 Jan; 24(1):91-8. Review.

McGarrigle D, Huang XY. GPCRs signaling directly through Src-family kinases. Sci STKE. 2007 Jun 26; 2007(392): pe35. Review.

McIntyre TM, Pontsler AV, Silva AR, St Hilaire A, Xu Y, Hinshaw JC, Zimmerman GA, Hama K, Aoki J, Arai H, Prestwich GD. Identification of an intracellular receptor for lysophosphatidic acid (LPA): LPA is a transcellular PPARgamma agonist. Proc Natl Acad Sci U S A. 2003 Jan 7; 100(1):131-6.

Meng Y, Kang S, Fishman DA. Lysophosphatidic acid inhibits anti-Fas-mediated apoptosis enhanced by actin depolymerization in epithelial ovarian cancer. FEBS Lett. 2005 Feb 14; 579(5):1311-9.

Milligan G, White JH. Protein-protein interactions at G-protein-coupled receptors. Trends Pharmacol Sci. 2001 Oct; 22(10):513-8. Review.

Mills GB, Moolenaar WH. The emerging role of lysophosphatidic acid in cancer. Nat Rev Cancer. 2003 Aug; 3(8):582-91. Review.

MOE. Montreal: Chemical Computing Group, 2002.

Moll UM, Wolff S, Speidel D, Deppert W. Transcription-independent pro-apoptotic functions of p53. Curr Opin Cell Biol. 2005 Dec; 17(6):631-6. Review. 
Moolenaar WH, van Meeteren LA, and Giepmans BN. The ins and outs of lysophosphatidic acid signaling. Bioessays. 2004 26:870-81.

Morris GM, Goodsell DS, Halliday RS, Huey R, Hart WE, Belew RK, and Olson AJ. Automated Docking Using a Lamarckian Genetic Algorithm and an Empirical Binding Free Energy Function. J Comput Chem. 1998 19:1639-62.

Nagata S. Fas-induced apoptosis, and diseases caused by its abnormality. Genes Cells. 1996 Oct; 1(10):873-9. Review.

Noguchi K, Ishii S, Shimizu T. Identification of p2y9/GPR23 as a novel G proteincoupled receptor for lysophosphatidic acid, structurally distant from the Edg family. J Biol Chem. 2003 Jul 11; 278(28):25600-6.

Oh YS, Jo NW, Choi JW, Kim HS, Seo SW, Kang KO, Hwang JI, Heo K, Kim SH, Kim YH, Kim IH, Kim JH, Banno Y, Ryu SH, Suh PG. NHERF2 specifically interacts with LPA2 receptor and defines the specificity and efficiency of receptor-mediated phospholipase C-beta3 activation. Mol Cell Biol. 2004 Jun; 24(11):5069-79.

Ohta H, Sato K, Murata N, Damirin A, Malchinkhuu E, Kon J, Kimura T, Tobo M, Yamazaki Y, Watanabe T, Yagi M, Sato M, Suzuki R, Murooka H, Sakai T, Nishitoba T, Im DS, Nochi H., Tamoto K, Tomura H, and Okajima F. Ki16425, a subtype-selective antagonist for EDG-family lysophosphatidic acid receptors. Mol Pharmacol. 2003 64:994-1005.

Parrill AL, Sardar VM, and Yuan H.. Sphingosine 1-phosphate and lysophosphatidic acid receptors: agonist and antagonist binding and progress toward development of receptorspecific ligands. Semin Cell Dev Biol. 2004 15:467-76.

Parrill AL, Wang D, Bautista DL, Van Brocklyn JR, Lorincz Z, Fischer DJ, Baker DL, Liliom K, Spiegel S, Tigyi G. Identification of Edg1 receptor residues that recognize sphingosine 1-phosphate. J Biol Chem. 2000 Dec 15; 275(50):39379-84.

Pasternack SM, von Kügelgen I, Aboud KA, Lee YA, Rüschendorf F, Voss K, Hillmer AM, Molderings GJ, Franz T, Ramirez A, Nürnberg P, Nöthen MM, Betz RC. G proteincoupled receptor P2Y5 and its ligand LPA are involved in maintenance of human hair growth. Nat Genet. 2008 Mar; 40(3):329-34.

Petit MM, Crombez KR, Vervenne HB, Weyns N, Van de Ven WJ. The tumor suppressor Scrib selectively interacts with specific members of the zyxin family of proteins. FEBS Lett. 2005 Sep 12; 579(22):5061-8.

Potten CS. Radiation, the ideal cytotoxic agent for studying the cell biology of tissues such as the small intestine. Radiat Res. 2004 Feb; 161(2):123-36. 
Prasad KV, Ao Z, Yoon Y, Wu MX, Rizk M, Jacquot S, Schlossman SF. CD27, a member of the tumor necrosis factor receptor family, induces apoptosis and binds to Siva, a proapoptotic protein. Proc Natl Acad Sci U S A. 1997 Jun 10; 94(12):6346-51.

Pritchard DM, Watson AJ. Apoptosis and gastrointestinal pharmacology. Pharmacol Ther. 1996 72:149-69.

Py B, Slomianny C, Auberger P, Petit PX, Benichou S. Siva-1 and an alternative splice form lacking the death domain, Siva-2, similarly induce apoptosis in T lymphocytes via a caspase-dependent mitochondrial pathway. J Immunol. 2004 Apr 1; 172(7):4008-17.

Qanbar R, Bouvier M. Role of palmitoylation/depalmitoylation reactions in G-proteincoupled receptor function. Pharmacol Ther. 2003 Jan; 97(1):1-33. Review.

Qin LF, Lee TK, Ng IO. Gene expression profiling by cDNA array in human hepatoma cell line in response to cisplatin treatment. Life Sci. 2002 Feb 22; 70(14):1677-90.

Quinlan MP, Douglas JL. Immortalization of primary epithelial cells requires first- and second-exon functions of adenovirus type 5 12S. J Virol. 1992 66(4):2020-30.

Radeff-Huang J, Seasholtz TM, Matteo RG, Brown JH. G protein mediated signaling pathways in lysophospholipid induced cell proliferation and survival. J Cell Biochem. 2004 Aug 1; 92(5):949-66. Review.

Raj GV, Sekula JA, Guo R, Madden JF, Daaka Y. Lysophosphatidic acid promotes survival of androgen-insensitive prostate cancer PC3 cells via activation of NF-kappaB. Prostate. 2004 Oct 1; 61(2):105-13.

Rajagopal K, Lefkowitz RJ, Rockman HA. When 7 transmembrane receptors are not G protein-coupled receptors. J Clin Invest. 2005 Nov; 115(11):2971-4. Review.

Resh MD. Palmitoylation of ligands, receptors, and intracellular signaling molecules. Sci STKE. 2006 Oct 31; 2006(359):re14. Review.

Roth AF, Wan J, Bailey AO, Sun B, Kuchar JA, Green WN, Phinney BS, Yates JR 3rd, Davis NG. Global analysis of protein palmitoylation in yeast. Cell. 2006a Jun 2; 125(5):1003-13.

Roth AF, Wan J, Green WN, Yates JR, Davis NG. Proteomic identification of palmitoylated proteins. Methods. 2006b Oct; 40(2):135-42. Review.

Rusovici R, Ghaleb A, Shim H, Yang VW, Yun CC. Lysophosphatidic acid prevents apoptosis of Caco-2 colon cancer cells via activation of mitogen-activated protein kinase and phosphorylation of Bad. Biochim Biophys Acta. 2007 Aug; 1770(8):1194-203. 
Sano T, Baker D, Virag T, Wada A, Yatomi Y, Kobayashi T, Igarashi Y, Tigyi G. Multiple mechanisms linked to platelet activation result in lysophosphatidic acid and sphingosine 1-phosphate generation in blood. J Biol Chem. 2002 Jun 14; 277(24):21197206.

Sardar VM, Bautista DL, Fischer DJ, Yokoyama K, Nusser N, Virag T, Wang de A, Baker DL, Tigyi G, Parrill AL. Molecular basis for lysophosphatidic acid receptor antagonist selectivity. Biochim Biophys Acta. 2002 1582:309-17.

Schmeichel KL, Beckerle MC. The LIM domain is a modular protein-binding interface. Cell. 1994 Oct 21; 79(2):211-9.

Schuler M, Green DR. Transcription, apoptosis and p53: catch-22. Trends Genet. 2005 Mar; 21(3):182-7. Review.

Schulte KM, Beyer A, Köhrer K, Oberhäuser S, Röher HD. Lysophosphatidic acid, a novel lipid growth factor for human thyroid cells: over-expression of the high-affinity receptor edg4 in differentiated thyroid cancer. Int J Cancer. 2001 Apr 15; 92(2):249-56.

Schultz DR, Harrington WJ Jr. Apoptosis: programmed cell death at a molecular level. Semin Arthritis Rheum. 2003 Jun; 32(6):345-69. Review.

Seta K, Nanamori M, Modrall JG, Neubig RR, Sadoshima J. 1 receptor mutant lacking heterotrimeric $\mathrm{G}$ protein coupling activates the Src-Ras-ERK pathway without nuclear translocation of ERKs. J Biol Chem. 2002 Mar 15; 277(11):9268-77.

Seta K, Sadoshima J. Phosphorylation of tyrosine 319 of the angiotensin II type 1 receptor mediates angiotensin II-induced trans-activation of the epidermal growth factor receptor. J Biol Chem. 2003 Mar 14; 278(11):9019-26.

Sheng M, Sala C. PDZ domains and the organization of supramolecular complexes. Annu Rev Neurosci. 2001 24:1-29. Review.

Shibue T, Taniguchi T. BH3-only proteins: integrated control point of apoptosis. Int J Cancer. 2006 Nov 1; 119(9):2036-43.

Shida D, Watanabe T, Aoki J, Hama K, Kitayama J, Sonoda H, Kishi Y, Yamaguchi H, Sasaki S, Sako A, Konishi T, Arai H, Nagawa H. Aberrant expression of lysophosphatidic acid (LPA) receptors in human colorectal cncer. Lab Invest. 2004 Oct; 84(10):1352-62.

Shimomura Y, Wajid M, Ishii Y, Shapiro L, Petukhova L, Gordon D, Christiano AM. Disruption of P2RY5, an orphan G protein-coupled receptor, underlies autosomal recessive woolly hair. Nat Genet. 2008 Mar; 40(3):335-9 
Solaz-Fuster MC, Gimeno-Alcaniz JV, Casado M, Sanz P. TRIP6 transcriptional coactivator is a novel substrate of AMP-activated protein kinase. Cell Signal. 2006 Oct; 18(10):1702-12.

Spierings D, McStay G, Saleh M, Bender C, Chipuk J, Maurer U, Green DR. Connected to death: the (unexpurgated) mitochondrial pathway of apoptosis. Science. 2005 Oct 7; 310(5745):66-7.

Steiner T. The Hydrogen Bond in the Solid State. Angew Chem Int Ed. 2002 41:48-76.

Sturm A, Dignass AU. Modulation of gastrointestinal wound repair and inflammation by phospholipids. Biochim Biophys Acta. 2002 1582:282-8.

Sun Y, Huang J, Xiang Y, Bastepe M, Jüppner H, Kobilka BK, Zhang JJ, Huang XY. Dosage-dependent switch from $\mathrm{G}$ protein-coupled to $\mathrm{G}$ protein-independent signaling by a GPCR. EMBO J. 2007 Jan 10; 26(1):53-64.

Sun Y, McGarrigle D, Huang XY. When a G protein-coupled receptor does not couple to a G protein. Mol Biosyst. 2007 Dec; 3(12):849-54. Review.

Suzuki Y, Nakabayashi Y, Takahashi R. Ubiquitin-protein ligase activity of X-linked inhibitor of apoptosis protein promotes proteasomal degradation of caspase- 3 and enhances its anti-apoptotic effect in Fas-induced cell death. Proc Natl Acad Sci U S A. $2001 \mathrm{Jul} 17$; 98(15):8662-7.

Tabata K, Baba K, Shiraishi A, Ito M, Fujita N. The orphan GPCR GPR87 was deorphanized and shown to be a lysophosphatidic acid receptor. Biochem Biophys Res Commun. 2007 Nov 23; 363(3):861-6.

Takizawa N, Smith TC, Nebl T, Crowley JL, Palmieri SJ, Lifshitz LM, Ehrhardt AG, Hoffman LM, Beckerle MC, Luna EJ. Supervillin modulation of focal adhesions involving TRIP6/ZRP-1. J Cell Biol. 2006 174(3):447-58.

Tigyi G. Physiological responses to lysophosphatidic acid and related glycerophospholipids. Prostaglandins Other Lipid Mediat. 2001 Apr; 64(1-4):47-62. Review.

Tigyi G, Parrill AL. Molecular mechanisms of lysophosphatidic acid action. Prog Lipid Res. 2003 Nov; 42(6):498-526. Review.

Tokumura A, Fukuzawa K, Akamatsu Y, Yamada S, Suzuki T, Tsukatani H. Identification of vasopressor phospholipid in crude soybean lecithin. Lipids 1978 13(7):468-72.

Tokumura A, Tsutsumi T, Tsukatani H. Transbilayer movement and metabolic fate of ether-linked phosphatidic acid (1-O-Octadecyl-2-acetyl-sn-glycerol 3-phosphate) in guinea pig peritoneal polymorphonuclear leukocytes. J Biol Chem. 1992 267:7275-83. 
Tsujimoto Y, Ikegaki N, Croce CM. Characterization of the protein product of bcl-2, the gene involved in human follicular lymphoma. Oncogene. 1987 2(1):3-7.

van der Bend RL, de Widt J, van Corven EJ, Moolenaar WH, van Blitterswijk WJ. Metabolic conversion of the biologically active phospholipid, lysophosphatidic acid, in fibroblasts. Biochim Biophys Acta. 1992 1125:110-12.

Velyvis A, Vaynberg J, Yang Y, Vinogradova O, Zhang Y, Wu C, Qin J. Structural and functional insights into PINCH LIM4 domain-mediated integrin signaling. Nat Struct Biol. 2003 Jul; 10(7):558-64.

Virag T, Elrod DB, Liliom K, Sardar VM, Parrill AL, Yokoyama K, Durgam G, Deng W, Miller DD, Tigyi G. Fatty alcohol phosphates are subtype-selective agonists and antagonists of lysophosphatidic acid receptors. Mol Pharmacol. 2003 May; 63(5):103242.

Wang DA, Lorincz Z, Bautista DL, Liliom K, Tigyi G, Parrill AL. A single amino acid determines lysophospholipid specificity of the S1P1 (EDG1) and LPA1 (EDG2) phospholipid growth factor receptors. J Biol Chem. 2001 Dec 28; 276(52):49213-20.

Wang Q, Liu M, Kozasa T, Rothstein JD, Sternweis PC, Neubig RR. Thrombin and lysophosphatidic acid receptors utilize distinct rhoGEFs in prostate cancer cells. J Biol Chem. 2004 Jul 9; 279(28):28831-4.

Wang Y, Gilmore TD. Zyxin and paxillin proteins: focal adhesion plaque LIM domain proteins go nuclear. Biochim Biophys Acta. 2003 Feb 17; 1593(2-3):115-20. Review.

Webb Y, Hermida-Matsumoto L, Resh MD. Inhibition of protein palmitoylation, raft localization, and $\mathrm{T}$ cell signaling by 2 -bromopalmitate and polyunsaturated fatty acids. $\mathrm{J}$ Biol Chem. 2000 Jan 7; 275(1):261-70.

Wei H, Ahn S, Shenoy SK, Karnik SS, Hunyady L, Luttrell LM, Lefkowitz RJ. Independent beta-arrestin 2 and $G$ protein-mediated pathways for angiotensin II activation of extracellular signal-regulated kinases 1 and 2. Proc Natl Acad Sci U S A. 2003 Sep 16; 100(19):10782-7.

Weiner JA, Chun J. Schwann cell survival mediated by the signaling phospholipid lysophosphatidic acid. Proc Natl Acad Sci U S A. 1999 Apr 27; 96(9):5233-8.

Xu J, Lai YJ, Lin WC, Lin FT. TRIP6 enhances lysophosphatidic acid-induced cell migration by interacting with the lysophosphatidic acid 2 receptor. J Biol Chem. 2004 Mar 12; 279(11):10459-68.

Xue L, Chu F, Cheng Y, Sun X, Borthakur A, Ramarao M, Pandey P, Wu M, Schlossman SF, Prasad KV. Siva-1 binds to and inhibits BCL-X(L)-mediated protection against UV radiation-induced apoptosis. Proc Natl Acad Sci U S A. 2002 May 14; 99(10):6925-30. 
Yamada T, Ohoka Y, Kogo M, Inagaki S. Physical and functional interactions of the lysophosphatidic acid receptors with PDZ domain-containing Rho guanine nucleotide exchange factors (RhoGEFs). J Biol Chem. 2005 280(19):19358-63.

Yamada T, Sato K, Komachi M, Malchinkhuu E, Tobo M, Kimura T, Kuwabara A, Yanagita Y, Ikeya T, Tanahashi Y, Ogawa T, Ohwada S, Morishita Y, Ohta H, Im DS, Tamoto K, Tomura H, Okajima F. Lysophosphatidic acid in malignant ascites stimulates motility of human pancreatic cancer cells through LPA1. J Biol Chem. 2004 Feb 20; 279(8):6595-605.

Yart A, Chap H, Raynal P. Phosphoinositide 3-kinases in lysophosphatidic acid signaling: regulation and cross-talk with the Ras/mitogen-activated protein kinase pathway. Biochim Biophys Acta. 2002 1582:107-11.

Ye X, Hama K, Contos JJ, Anliker B, Inoue A, Skinner MK, Suzuki H, Amano T, Kennedy G, Arai H, Aoki J, Chun J. LPA3-mediated lysophosphatidic acid signalling in embryo implantation and spacing. Nature. 2005 May 5; 435(7038):104-8.

Ye X, Ishii I, Kingsbury MA, Chun J. Lysophosphatidic acid as a novel cell survival/apoptotic factor. Biochim Biophys Acta. 2002 Dec 30; 1585(2-3):108-13. Review.

Yi J, Beckerle MC. The human TRIP6 gene encodes a LIM domain protein and maps to chromosome 7q22, a region associated with tumorigenesis. Genomics. 1998 49(2):314-6.

Yu GY, Lee KJ, Gao L, Lai MM. Palmitoylation and polymerization of hepatitis C virus NS4B protein. J Virol. 2006 Jun; 80(12):6013-23.

Yue J, Yokoyama K, Balazs L, Baker DL, Smalley D, Pilquil C, Brindley DN, Tigyi G. Mice with transgenic overexpression of lipid phosphate phosphatase-1 display multiple organotypic deficits without alteration in circulating lysophosphatidate level. Cell Signal. 2004 16:385-99.

Yun CC, Sun H, Wang D, Rusovici R, Castleberry A, Hall RA, Shim H. LPA2 receptor mediates mitogenic signals in human colon cancer cells. Am J Physiol Cell Physiol. 2005 289(1): C2-11.

Zhai P, Yamamoto M, Galeotti J, Liu J, Masurekar M, Thaisz J, Irie K, Holle E, Yu X, Kupershmidt S, Roden DM, Wagner T, Yatani A, Vatner DE, Vatner SF, Sadoshima J. Cardiac-specific overexpression of AT1 receptor mutant lacking $\mathrm{G}$ alpha $\mathrm{q} / \mathrm{G}$ alpha $\mathrm{i}$ coupling causes hypertrophy and bradycardia in transgenic mice. J Clin Invest. 2005 Nov; 115(11):3045-56.

Zhang H, Wang D, Sun H, Hall RA, Yun CC. MAGI-3 regulates LPA-induced activation of Erk and RhoA. Cell Signal. 2007 Feb; 19(2):261-8. 
Zhao MK, Wang Y, Murphy K, Yi J, Beckerle MC, Gilmore TD. LIM domaincontaining protein trip6 can act as a coactivator for the v-Rel transcription factor. Gene Expr. 1999 8(4): 207-17.

Zheng Q, Zhao Y. The diverse biofunctions of LIM domain proteins: determined by subcellular localization and protein-protein interaction. Biol Cell. 2007 Sep; 99(9):489502. Review.

Zheng Y, Voice JK, Kong Y, Goetzl EJ. Altered expression and functional profile of lysophosphatidic acid receptors in mitogen-activated human blood $\mathrm{T}$ lymphocytes. FASEB J. 2000 Dec; 14(15):2387-9. 


\section{VITA}

Shuyu E was born on February 6, 1972 in People's Republic of China. She got her M.D. in 1996 and Master of Science in Pharmacology major in 1999. She joined in the Physiology program in University of Tennessee Health Science Center in Memphis for her Ph.D. in August of 2001. She completed her doctorate degree in December of 2008. 